\title{
A BDNF-TrkB autocrine loop enhances senescent cell viability
}

\section{Carlos Anerillas}

Laboratory of Genetics and Genomics, National Institute on Aging https://orcid.org/0000-0003-44247913

Allison Herman

$\mathrm{NIH}$

\section{Rachel Munk}

National Institute on Aging

\section{Matthew Payea}

$\mathrm{NIH}$

Martina Rossi

$\mathrm{NIH}$

Dimitrios Tsitsipatis

$\mathrm{NIH}$

Jennifer Martindale

Yulan Piao

National Institute on Aging, $\mathrm{NIH}$

Krystyna Mazan-Mamczarz

$\mathrm{NIH}$

Jinshui Fan

$\mathrm{NIH}$

Chang-Yi Cui

$\mathrm{NIH}$

\section{Supriyo De}

National Institute on Aging, $\mathrm{NIH}$

Kotb Abdelmohsen

National Institute on Aging https://orcid.org/0000-0001-6240-5810

Rafael de Cabo

National Institute on Aging, NIH https://orcid.org/0000-0002-3354-2442

Myriam Gorospe ( $\sim$ gorospem@grc.nia.nih.gov )

National Institutes of Health https://orcid.org/0000-0001-5439-3434 


\section{Keywords:}

Posted Date: January 25th, 2022

DOI: https://doi.org/10.21203/rs.3.rs-1263555/v1

License: (c) (1) This work is licensed under a Creative Commons Attribution 4.0 International License. Read Full License

Version of Record: A version of this preprint was published at Nature Communications on October 20th, 2022. See the published version at https://doi.org/10.1038/s41467-022-33709-8. 


\section{A BDNF-TrkB autocrine loop enhances senescent cell viability}

Carlos Anerillas ${ }^{1, \star}$, Allison B. Herman ${ }^{1}$, Rachel Munk ${ }^{1}$, Matthew J. Payea ${ }^{1}$, Martina Rossi ${ }^{1}$, Dimitrios Tsitsipatis ${ }^{1}$, Jennifer L. Martindale ${ }^{1}$, Yulan Piao', Krystyna Mazan-Mamczarz ${ }^{1}$, Jinshui Fan', Chang-Yi Cui ${ }^{1}$, Supriyo De ${ }^{1}$, Kotb Abdelmohsen', Rafael de Cabo ${ }^{2}$, and Myriam Gorospe ${ }^{1, *}$

${ }^{1}$ Laboratory of Genetics and Genomics and ${ }^{2}$ Translational Gerontology Branch, National Institute on Aging Intramural Research Program, National Institutes of Health, Baltimore, Maryland, USA

*Co-corresponding authors:

Laboratory of Genetics and Genomics

NIA IRP, NIH

251 Bayview Blvd.

Baltimore, MD 21224, USA

Tel: +1 410454 8412; Email: myriam-gorospe@nih.gov; carlos.anerillasaljama@nih.gov

Running title: BDNF-TrkB signaling supports senescent cells 
Cellular senescence is characterized by cell cycle arrest, resistance to apoptosis, and a senescence-associated secretory phenotype (SASP) whereby cells secrete pro-inflammatory and tissue-remodeling factors. Given that the SASP exacerbates age-associated pathologies, some aging interventions aim at selectively eliminating senescent cells. In this study, a drug library screen uncovered TrkB (NTRK2) inhibitors selectively capable of triggering apoptosis of senescent, but not proliferating, human fibroblasts. Senescent cells expressed high levels of TrkB, which supported senescent cell viability, and secreted the TrkB ligand BDNF. The reduced viability of senescent cells after ablating BDNF signaling suggested an autocrine function for TrkB and BDNF, including increased expression of BCL2L2 downstream of BDNF-TrkB, favoring senescent cell survival. Strikingly, treatment with TrkB inhibitors reduced the accumulation of senescent cells in aged mouse organs. We propose that the activation of TrkB by SASP factor BDNF promotes cell survival and could be exploited therapeutically to reduce the senescent-cell burden. 
The cell senescence program is implemented in response to different forms of non-lethal cell damage. Senescent cells are characterized by several traits, including morphological changes, indefinite growth arrest, and metabolic reprogramming that includes altered lysosomal function with increased presence of a senescence-associated $\beta$-Galactosidase (SA- $\beta$-Gal) activity ${ }^{1}$. However, one of their most prominent features is the senescence-associated secretory phenotype (SASP), a trait defined as the active secretion of pro-inflammatory factors and matrix-remodeling enzymes. Through these secreted proteins, senescent cells modify their environment and influence physiologic and disease processes ${ }^{2}$.

On the one hand, senescence has been identified as critically beneficial for tissue repair, embryonic development, and tumor suppression; on the other, the uncontrolled accumulation of senescent cells within organs can lead to tissue dysfunction and disease ${ }^{3}$. With advancing age, senescent cells increase in tissues and organs, exacerbating a range of aging-related physiologic declines and diseases ${ }^{4}$. The pharmacologic removal of senescent cells using senolytics in mouse models was recently found to improve several age-associated disorders ${ }^{5}$. Many senolytic strategies exploit the fact that senescent cells exist in a state of potent suppression of apoptosis ${ }^{6}$, for instance, by inhibiting the pro-survival effect of anti-apoptotic proteins in the BCL2 family ${ }^{7,8}$.

A range of roles have been identified for mitogen-activated protein kinase (MAPK) signaling pathways in senolysis 9 . To address the influence of MAPKs experimentally and systematically, we employed a chemical library including inhibitors of every step in the MAPK cascade, from the receptors down to the final effector proteins. This survey identified two drugs, both inhibitors of tropomyosin receptor kinase (Trk) activity, that effectively and specifically reduced the viability of senescent WI-38 and BJ human diploid fibroblasts. Trk receptors are best known for transducing signals from their known ligands, the neurotrophins, to modulate neuronal development and communication $^{10}$. Among the Trk family, which includes TrkA, TrkB, and TrkC, we identified TrkB as being primarily responsible for maintaining senescent cell viability in this unexpected context. Further interrogation revealed that cells rendered senescent in different ways secrete the neurotrophin BDNF (brain-derived neurotrophic factor), and that in cultured fibroblasts, autocrine/paracrine activation of TrkB by BDNF sustained ERK5 activation and BCL2L2-dependent viability of senescent cells. We present evidence that inhibiting this regulatory paradigm reduces the senescent cell burden in mouse tissues, and propose that suppressing the signaling through TrkB and BDNF can be exploited to eliminate senescent cells for therapeutic benefit. 


\section{Results}

\section{MAPK-directed drug screen identifies inhibitors of Trk receptors as potential senolytic drugs}

As discussed recently ${ }^{9}$, the role of MAPK pathways in senescence-associated apoptosis is poorly understood. To better understand how targeting MAPKs in senescence might offer therapeutic benefit, we screened a custom library (Tocris) comprising 43 compounds that inhibit different mediators of MAPK signaling cascades (Fig. 1a, Supplementary Table 1). The screen included a known senolytic, ABT-737, as a positive control, and was carried out in parallel on proliferating cells to ensure that the identified drugs selectively affected senescent cells. To strengthen our screen, we performed it on two human diploid fibroblasts lines (WI-38 and BJ) that are well-established in senescence studies. The experimental conditions were previously optimized to trigger cellular senescence in both cell types with minimal cell death. Briefly, WI-38 and BJ fibroblasts were treated with 50 or $25 \mu \mathrm{M}$ etoposide, respectively, refreshing the drug every three days. After ten days, when cells had reached senescence, etoposide was removed, and the different compounds from the library were tested for an additional 48 hours (Fig. 1b). The induction of senescence in WI-38 and BJ cells was confirmed by measuring senescence-associated beta-Galactosidase (SA- $\beta$-Gal) activity (Extended Data Fig. 1a-d); growth arrest in the senescent cultures was confirmed by measuring BrdU incorporation (Extended Data Fig. 1e,f).

Next, we assessed the effect of treatment with the drugs in the library (each at $10 \mu \mathrm{M}$ ) for $48 \mathrm{~h}$ on both proliferating and senescent WI-38 or BJ cells. The impact of each drug was evaluated by direct cell counts at the end of the treatment relative to the initial cell numbers. As shown in Fig. 1c (further information can be found in Supplementary Table 1), treatment with several inhibitors caused specific decreases in the number of live senescent cells; the heat map (Fig. 1d) represents the percentage of senolysis caused by a certain drug (proportion of dead cells $48 \mathrm{~h}$ later). The drugs were grouped by the target proteins within the MAPK pathways upon which they act. We excluded from the heat map those drugs that decreased the viability of proliferating cells, such as CMPD-1 (an inhibitor of MK2 and microtubular assembly), BRD 7389 (a p90 S6K inhibitor), HI TOPK 032 (a TOPK inhibitor), and AC 710 (a PDGFR inhibitor). Strikingly, two of the drugs with the most pronounced senolytic effect were the TrkB receptor inhibitors GNF 5837 and ANA 12 (blue arrows in Fig. 1c,d). TrkB belongs to a family of Receptor Tyrosine Kinases (RTKs) whose ligands are neurotrophins, a group of secreted proteins crucial for engaging neuronal survival ${ }^{11}$. Although GNF 5837 also inhibits TrkA and TrkC, other inhibitors included in the library selectively targeting other members of this family, such as TrkA (GW 441756) and NGFR (PD 90780), did not show any senolytic properties. Thus, in this screen, TrkB appeared to be the main Trk receptor responsible for maintaining the viability of senescent cells. Together, these data indicate that TrkB inhibitors have potential senolytic effects. 
Anerillas et al. page 5

\section{Pharmacological inhibition of TrkB selectively induces apoptotic cell death of senescent cells}

We sought to explore further the ability of Trk inhibitors to selectively induce apoptosis in senescent cells. In addition to GNF 5837 and ANA 12, our analysis included PF 06273340, an additional Trk inhibitor that does not cross the blood-brain barrier $(\mathrm{BBB})^{12}$. Given that Trk receptor activity is essential for cognitive function ${ }^{11}$, drugs incapable of crossing the BBB, such as GNF 5837 and PF $06273340^{12}$ would have less cognitive side-effects and thus might be more attractive for therapy. ANA 12 does enter the central nervous system, and it has been proposed to be beneficial as an antidepressant $^{13}$.

Therefore, we evaluated a range of doses of each drug $(1.25$ to $50 \mu \mathrm{M})$ in proliferating and senescent cells (Fig. 2a). Analysis of viability after $48 \mathrm{~h}$ later revealed that the optimal senolytic doses are $10 \mu \mathrm{M}$ GNF 5837, $20 \mu \mathrm{M}$ ANA 12, and $30 \mu \mathrm{M}$ PF 06273340 (Fig. 2b,c), since they caused maximal death of senescent cells with minimal consequences on proliferating cells. These doses also significantly increased Caspase 3/7 activity, an indicator of apoptosis, in senescent cells relative to untreated senescent cells (Extended Data Fig. 2, Fig. 2d) as tested previously ${ }^{14}$. Importantly the same doses effectively reduced the viability of WI-38 cells that had been rendered senescent by other means -replicative senescence (RS) or ionizing radiation-induced senescence (IRIS) (Fig. 2e; Extended Data Fig. 2b-d). We further tested their effectiveness in reducing cell viability in other senescence models, including BJ or IMR-90 fibroblasts rendered senescent by treatment with etoposide. After confirming the induction of senescence in these models by measuring SA- $\beta$-Gal activity and BrdU incorporation (Extended Data Fig. 2b-d), we evaluated the effects of treatments with $10 \mu \mathrm{M}$ GNF 5837, $20 \mu \mathrm{M}$ ANA 12, or $30 \mu \mathrm{M}$ PF 06273340 . Importantly, while these doses did not reduce the viability of proliferating cells (Extended Data Fig. 2e), they strongly induced death of both senescent BJ and IMR90 cells (Fig. 2f). In keeping with the results seen in senescent WI-38 cells, senescent BJ and IMR-90 fibroblasts displayed elevated caspase activity in the presence of Trk inhibitors (Fig. 2g), supporting the notion that these cells experienced increased apoptotic death in response to these treatments.

Finally, to confirm the role of apoptosis in the death caused by TrkB inhibitors, we blocked caspase activity by incubating cells with the pan-caspase inhibitor Z-VAD-FMK along with GNF 5837, ANA 12, or PF 06273340 . As shown, the increased cell death and caspase 3/7 activity seen by inhibiting Trk (Fig. 2f,g) were reversed when cells were simultaneously treated with Z-VAD-FMK (Figs. 2h,i). Together, these data support the notion that inhibiting Trk enhanced pro-apoptotic signaling in senescent cells, leading to cell death.

\section{Elevated TrkB levels in senescent cells promotes resistance to apoptosis}

We then set out to further characterize the role of Trk receptors in cellular senescence. In neuronal cells, activation of Trk receptors strongly promotes cell viability through specific gene expression programs ${ }^{15}$. Although our data pointed to a prominent pro-survival role for TrkB in senescent cells, we 
also analyzed the structurally similar TrkA and TrkC. We first assessed the levels of NTRK1, NTRK2, and NTRK3 mRNAs (encoding TrkA, TrkB, and TrkC, respectively), along with the levels of p16/CDKN2A and IL6 mRNAs, two markers of senescence, by reverse transcription (RT) followed by real-time quantitative (q)PCR analysis. Although we were unable to detect NTRK3 mRNA in WI-38 fibroblasts, the levels of NTRK1 and NTRK2 mRNAs increased slightly, while p16 and IL6 mRNAs increased markedly, as expected, in senescent $(\mathrm{S})$ relative to proliferating $(\mathrm{P})$ cells (Fig. 3a). By western blot analysis, however, the levels of TrkA, TrkB, and senescence marker p21 increased robustly in the senescent populations, including WI-38, BJ, and IMR-90 cells rendered senescent by treatment with etoposide, and replicatively senescent (RS) WI-38 cells (Fig. 3b).

To determine the role of each Trk receptor in senescent cell viability, we reduced the levels of TrkA or TrkB by transfecting specific small interfering (si)RNAs. After silencing was achieved, we triggered senescence with etoposide and monitored cell viability. As observed in Fig. 3c, cells in which TrkA was silenced (siNTRK1) and control cells did not show reduced viability, while silencing TrkB (siNTRK2) significantly decreased the numbers of live cells. The efficiency of the silencing interventions was confirmed by measuring mRNA levels (Fig. 3d); silencing of TrkA or TrkB in proliferating cells did not affect viability (not shown). In sum, despite the rise in TrkA levels in some senescence models, the enhanced viability of senescent cells appears to be mediated by TrkB.

Next, since TrkB protein levels increased proportionally more than NTRK2 mRNA levels in senescent cells, we evaluated the stability of TrkB protein by treating proliferating and senescent cells with cycloheximide (CHX), an inhibitor of de novo translation. This analysis included positive control p53 (TP53), as its stability increases in senescent cells ${ }^{16,17}$. As assessed by western blot analysis (Fig. 3e), TrkB appeared more stable in senescent fibroblasts, as did p53, suggesting that increased TrkB protein stability contributes to its accumulation in senescent cells. A time-course analysis after triggering senescence with etoposide revealed that TrkB protein levels increased by day 2 and continued to rise until day 10 (Fig. 3f). Early markers of senescence, p21 protein and TGFB1 mRNA (Figs. 3f,g) ${ }^{18-20}$, as well as late markers of senescence, pl6 and IL6 mRNAs 1 (Fig. 3g), were included in the analysis. Although with different kinetics, TrkB levels also increased progressively with senescence in BJ and IMR-90 cells (Extended Data Fig. 3). Finally, we tested if TrkB was found on the plasma membrane of senescent cells. We enriched plasma surface proteins using a biotinylation strategy (Methods) and found that TrkB was present on the membrane of senescent cells; we included as a positive control DPP4, a protein previously reported to increase on the plasma membrane of senescent cells ${ }^{21}$ (Fig. 3h). Immunofluorescence detection of TrkB using non-permeabilizing conditions confirmed that TrkB was strongly abundant on the membrane of senescent cells, as was DPP4 (Fig. 3i). Together, these data indicate that TrkB protein increases during senescence in several cell systems, and contributes to ensuring senescent cell viability. 


\section{BDNF is a novel SASP member that ensures viability in senescent cells}

Trk receptors are activated by a family of secreted ligands known as neurotrophins, comprising NGF, NTF3, NTF4, and BDNF ${ }^{15}$, which are essential for neuronal function. Even though BDNF is the preferred ligand of TrkB, we explored possible roles for all neurotrophins in this paradigm of cell senescence. First, by RT-qPCR analysis, we measured the levels of NGF, NTF3, NTF4, and BDNF mRNAs in WI-38, BJ, and IMR-90 fibroblasts induced to undergo senescence by etoposide treatment for ten days. Interestingly, only $B D N F$ mRNA was consistently elevated in the three senescence models (Fig. 4a). Second, we found increased BDNF mRNA levels in transcriptomic datasets obtained from different models of senescence: in WI-38 cells rendered senescent by replicative exhaustion or by treatment with doxorubicin or IR; as well as in human umbilical vein endothelial cells (HUVEC) and human alveolar epithelial cells (HAEC) cells rendered senescent by treatment with IR (Extended Data Fig. 4a $)^{22}$. Third, to test if BDNF is secreted by senescent cells, we collected media from the senescence models used in Fig. 4a; as shown, the levels of secreted BDNF, as analyzed by ELISA, were significantly higher in media collected from senescent cells compared to proliferating cells (Fig. 4b); NGF, NTF3, and NTF4 were undetectable by ELISA in either population (Extended Data Fig. 4b). Increased levels of BDNF and other SASP cytokines ([CXCL1 (GRO-alpha), IL6, HGF, or CXCL10] were also detected in media from senescent cells by using a cytokine array (Fig. 4c). NTF3 and NTF4 were included in this array but did not increase in the media obtained from senescent cells. Together, these data indicate that senescent cells secrete BDNF.

By immunofluorescence microscopy, we found that senescent WI-38 cells were strongly positive for BDNF (Fig. 4d). RT-qPCR analysis likewise revealed a rise in BDNF mRNA levels two days after triggering senescence with etoposide (Fig. 4e). Since many SASP members are regulated by $\mathrm{NF}-\kappa \mathrm{B}$ or $\mathrm{p} 53$, we investigated if these two transcription factors induced BDNF production in senescent cells. Following transfection with siRNAs directed at TP53 and RELA mRNAs to silence p53 and RELA, respectively, we observed that the rise in BDNF mRNA levels in senescent cells was dramatically suppressed by silencing p53 and modestly reduced by silencing RELA (Fig. 4f). The senescence-associated rise in $p 21$ mRNA levels, which is dependent on the transcriptional activity of p53, was similarly reduced by silencing p53, while induction of the senescence-associated induction in IL6 mRNA, an NF- $\mathrm{BB}$-regulated mRNA, was mostly abrogated by silencing RELA. The rise in p16 mRNA was not reversed by either silencing intervention.

We then asked if BDNF might directly affect senescent cell survival in a manner similar to how it promotes neuronal viability, through TrkB activation ${ }^{15,23}$. First, we silenced BDNF by using specific siRNAs directed at BDNF mRNA (Ctrl siRNAs were used in control transfections), and then we triggered senescence with etoposide. As shown in Fig. 4g, silencing BDNF before triggering senescence significantly reduced cell viability relative to control ( $\mathrm{siCtrl}$ ) cells that reached senescence but were able to express BDNF. Importantly, BDNF silencing did not influence the survival of 
Anerillas et al. page 8

proliferating cells, suggesting that it selectively promoted the survival of senescent cells; RT-qPCR analysis confirmed the silencing interventions (Fig. 4h). To further assess if the effect of BDNF was autocrine/paracrine, we employed blocking antibodies. Forty-eight hours after adding anti-BDNF or non-specific IgG antibodies $(4 \mu \mathrm{g} / \mathrm{ml})$ to the media, proliferating cells showed no change in proliferation or viability, while senescent cells showed markedly reduced viability in the presence of anti-BDNF antibodies (Figs. 4i,j). Moreover, caspase 3/7 activity increased by blocking BDNF with antibodies (Fig. 4k). In sum, our results identify BDNF as a SASP member implicated in promoting senescent cell survival and further indicate that BDNF secreted by senescent cells promotes viability in an autocrine and/or paracrine fashion.

\section{ERK5 activation by TrkB-BDNF axis sustains senescent cell survival through BCL2L2}

We then sought to investigate if the activation of TrkB by BDNF was linked to the TrkB-mediated survival of senescent cells. First, to visualize protein phosphorylation we employed phos-tag polyacrylamide gels, which capture phosphorylated proteins and reduce their migration, and compared the proportion of slow-migrating bands (phosphorylated) to those migrating at the expected size (nonphosphorylated). As shown, slow-migrating TrkB bands appeared by 2 to 3 days of etoposide treatment, increasing in intensity in subsequent days (Fig. 5a, left); notably, the dynamics of the rise in phosphorylation mirrored the rise in BDNF mRNA abundance. To test if the two processes were related, we silenced BDNF before triggering senescence and found that this intervention completely prevented the appearance of the phosphorylated TrkB band 6 days later (Fig. 5a, right). These data suggest that the production of BDNF is critical for inducing TrkB phosphorylation in senescent cells. Second, we tested whether both TrkB and BDNF depletion affected senescent cell viability in a similar time frame. Given that TrkB and BDNF levels rose markedly by 2 days into senescence, and that silencing either TrkB or BDNF increased cell death, we evaluated whether their depletion affected senescent cell survival at early and/or late senescence, two stages defined previously ${ }^{19,24}$. We found that silencing TrkB or BDNF decreased cell survival at both early and late senescence, although the survival was more strongly reduced in late senescence (Fig. $\mathbf{5 b}$ ).

To further explore the ability of BDNF and TrkB to modulate senescent cell survival, we sequenced the bulk RNA present in senescent cells in which either TrkB or BDNF were silenced, and compared it to siCtrl-transfected senescent counterparts (Fig. 5c). The RNA-seq data are deposited in GSE190998 (https://www.ncbi.nlm.nih.gov/geo/query/acc.cgi?acc=GSE190998; reviewer token crqxgaculzcxnid). GSEA analysis of RNAs differentially abundant after silencing TrkB or BDNF revealed enriched pathways of apoptosis and caspase activity (Fig. 5d, left). The heat map in Fig. 5d, comprising differentially abundant mRNAs encoding apoptosis-related proteins, revealed jointly modulated transcripts, including BCL2L2 mRNA, which encodes a protein essential for the survival of senescent cells ${ }^{8,25}$. We used RT-qPCR analysis to validate these results by measuring the levels of 
$B C L 2 L 2$ mRNA, along with the levels of other mRNAs encoding apoptosis-modulating proteins such as BCL2L1 and PUMA ${ }^{6,8}$, and included conditions in which TrkB or BDNF were silenced (Extended Data Fig. 5a). Notably, while the levels of p53-induced pro-apoptotic PUMA mRNA were not affected, the levels of BCL2L2 mRNA, encoding the anti-apoptotic protein BCL2L2, decreased after TrkB or BDNF silencing at both early and late senescence (Fig. 5e). This effect appeared to be specific for BCL2L2 mRNA, as BCL2L1 mRNA levels were unchanged after silencing TrkB or BDNF (Fig. 5e), as were the levels of the p53-regulated $p 21$ mRNA, while IL6 mRNA levels unexpectedly decreased after silencing TrkB or BDNF (Extended Data Fig. 5a). Furthermore, BDNF-blocking antibodies specifically blunted the rise in BCL2L2 mRNA in senescent cells, while PUMA and BCL2L1 mRNAs were unaffected (Fig. 5f). As seen in TrkB- or BDNF-silenced senescent cells (Extended Data Fig. 5a), p21 mRNA levels did not change in BDNF-blocked cells while IL6 mRNA levels decreased after treatment with BDNF-blocking antibodies (Extended Data Fig. 5b). These results were confirmed by western blot analysis of BCL2L2 (BCLW) expression levels; p53 levels were unchanged (Fig. 5g).

Next, we analyzed the pathways downstream of BDNF and TrkB signaling. Phosphorylation of ERK5 was dramatically reduced by silencing TrkB or BDNF, in both early and late senescence (Fig. 5h), a notable finding, given that ERK5 elevates BCL2L2 levels downstream of BDNF-TrkB for the survival of neuronal cells ${ }^{26}$. We then utilized a highly selective ERK5 inhibitor (ERK5-IN-1, $\left.1 \mu \mathrm{M}\right)$ to reduce ERK5 activity in senescent cells and found that it lowered both BCL2L2 levels and senescentcell viability (Figs. 5i,j and Extended Data Fig. 5c), but it did not affect proliferating cells (Extended Data Fig. 5d). Again, in agreement with the results after silencing TrkB or BDNF (Extended Data Fig. 5a), inhibiting ERK5 specifically lowered the levels of BCL2L2 and IL6 mRNAs, but not the levels of PUMA, BCL2L1, or p21 mRNAs (Fig. 5i and Extended Data Fig. 5E). In sum, our data suggest that activation of TrkB by BDNF specifically activates ERK5, which in turn increases BCL2L2 levels.

\section{BDNF is a discrete marker of survival in cellular senescence}

In light of our earlier findings that p53 increases BDNF production, we investigated if BDNF induction contributed to the actions of p53 in senescence. First, we treated WI-38 cells with a range of doses of etoposide that had different effects on cell proliferation and viability. Untreated cells proliferated as expected, but increasing doses of etoposide reduced proliferation to different degrees ( 5 to $50 \mu \mathrm{M}$ etoposide) or caused cell death (100 to $200 \mu \mathrm{M}$ etoposide) (Fig. 6a). BDNF mRNA levels increased at etoposide concentrations that reduced proliferation $(\leq 50 \mu \mathrm{M})$ but not at concentrations that triggered cell death (100-200 $\mu \mathrm{M})$ (Fig. 6b); by contrast, p21 and IL6 mRNAs accumulated with increasing DNA damage (Fig. 6b). Second, we modulated p53 function by silencing and chemical activation to study if p53 influenced BDNF mRNA levels. As shown in Fig. 6c, silencing p53 prevented the rise in BDNF mRNA elicited by $50 \mu \mathrm{M}$ etoposide treatment for $48 \mathrm{~h}$; the same interventions also reduced $p 21 \mathrm{mRNA}$ but not IL6 mRNA (Extended Data Fig. 6a,b). These experiments underscore a requirement for 
Anerillas et al. page 10

functional p53 to induce BDNF even at early stages of senescence, despite the absence of p53 sites on the $B D N F$ promoter. We then tested if increasing p53 affected BDNF levels at a senescence-inducing dose of etoposide $(50 \mu \mathrm{M})$ by employing Nutlin-3a (Nut3a), a p53-stabilizing compound ${ }^{27}$. Notably, increasing p53 in cells treated with $50 \mu \mathrm{M}$ etoposide significantly decreased BDNF levels (Fig. 6c), resembling the reduction observed at higher levels of DNA damage; $p 21 \mathrm{mRNA}$ levels increased in the presence of Nut3a (Extended Data Fig. 6c) and Nut3a reduced cell viability (Extended Data Fig. 6d). These data suggest that only moderate p53 levels induce BDNF production; levels of p53 that are too low or too high prevent the rise in BDNF that promotes survival.

Several mechanisms can explain how p53 could both induce and repress BDNF production depending on the extent of DNA damage. STAT3 was recently reported to promote BDNF expression in the lung upon injury with $\operatorname{acid}^{28}$ and we found that phosphorylation of STAT3 at Tyrosine 705 decreased in IMR-90 fibroblasts committed to apoptosis but remained phosphorylated in senescencecommitted cells (Anerillas et al., submitted). Therefore, we silenced STAT3 by transfection with siRNA (Extended Data Fig. 6e) and evaluated BDNF induction by $50 \mu \mathrm{M}$ etoposide treatment $48 \mathrm{~h}$ later. As shown in Fig. 6d, the rise in BDNF mRNA triggered by senescence-inducing etoposide (50 $\mu \mathrm{M})$ was prevented by STAT3 silencing; by contrast, $p 21$ mRNA levels were not affected and the slight rise in IL6 mRNA was suppressed (Extended Data Fig. 6e). Despite the observation that silencing STAT3 lowered BDNF levels, silencing STAT3 did not reduce cell viability, suggesting that STAT3 might be part of multiple factors governing the survival-apoptosis balance in early senescence (Extended Data Fig. 6f). Interestingly, both p53 silencing and p53 activation decreased STAT3 phosphorylation (Fig. 6e), mirroring the effects seen for BDNF. Over the doses tested, p53 levels increased linearly along with DNA damage, but STAT3 phosphorylation at Tyr705 peaked at $25 \mu \mathrm{M}$, when p53 levels were moderately elevated. Finally, we evaluated whether both p-STAT3 and BDNF were simultaneously activated at a single-cell level during senescence by double immunofluorescence staining of senescent WI-38 cells with antibodies recognizing p-STAT3(Tyr705) and BDNF. Interestingly most BDNF-positive cells were p-STAT3(Tyr705)-positive, but virtually every pSTAT3(Tyr705)-positive cell was BDNF-positive (Fig. 6g,h). Together, these experiments indicate that p53 can both induce or repress BDNF depending on the level of DNA damage and that pSTAT3(Tyr705) critically mediates BDNF induction in senescence. In turn, BDNF secretion by senescent cells can trigger signaling through TrkB $\rightarrow$ ERK5 $\rightarrow$ BCL2L2 to ensure survival in an autocrine manner (Fig. 6i).

\section{Delivery of TrkB inhibitors reduces senescent cell burden in old mice}

Finally, we tested the relevance of the BDNF-TrkB paradigm on senescent cell viability in vivo using C57BL/6J mice. We chose naturally aged mice as a well-established and relevant in vivo cellular senescence model ${ }^{29}$. The appearance of senescent cells in older tissues such as lung and liver has been 
Anerillas et al. page 11

documented by different methods, such as assessment of SA- $\beta$-Gal activity or immunostaining for senescence markers like p16 $6^{30-32}$. Initially, we studied the extent to which p16- and BDNF-positive cells colocalized in tissues in 24 month-old (m.o.) mice. Interestingly, one-half of the p16-positive cells in lung and liver were also BDNF-positive (Fig. 7a), suggesting that the two proteins were coexpressed in aging.

We then tested the ability of Trk inhibitors to reduce cell senescence markers in old mice. We administered the Trk inhibitors identified in Fig. 1 by monthly injections into 21 m.o. mice until they were 24 m.o. (Fig. 7b and extended information in Methods). We assayed the same inhibitors we had tested in culture (GNF 5837, ANA 12, and PF 06273340), but halted treatments with ANA 12 due to toxicity; in fact, ANA 12 was a poor candidate for therapy due to its ability to penetrate the $\mathrm{BBB}^{13}$. Compared with control mice [vehicle-treated 24 m.o. and untreated young (3 m.o.) mice], GNF 5837 and PF 06273340 had promising effects on senescence markers and traits. The increases in levels of p16 and p21 mRNAs observed in kidney, lung, and liver of old mice were significantly mitigated by each drug (Fig. 7c); the same trends were seen for $I l 6$ and $I l l b$ mRNAs, but they did not reach significance in all organs (Fig. 7c). These observations indicate that these drugs, which do not cross the $\mathrm{BBB}$, were able to decrease the levels of prominent senescence markers such as p16 or $p 21$ mRNAs in several tissues in aged mice.

We then studied senescence-related serum markers that were recently found to increase with age $^{33}$. One such marker, GDF15, increased markedly in old mice; this increase was significantly mitigated by treatment with PF 06273340 , while GNF 5837 showed the same trend without reaching significance (Fig. 7d). Other serum markers (TIMP-1 and PAI-1) were not elevated with age (Extended Data Fig. 7a) and thus they could not be used to test the effects of these drugs. In addition, the drugs did not affect body weight (Extended Data Fig. 7b). Immunofluorescence analysis revealed that both GNF 5837 and PF 06273340 treatments reduced the percentage of p16-positive cells in all three organs tested (Fig. 7e and Extended Data Fig. 7c). As was the case for GDF15 serum levels, PF 06273340 appeared to be more effective than GNF 5837 in reducing p16 levels.

Finally, we assessed the canonical SA- $\beta$-Gal marker in three organs. Remarkably, both drug treatments reduced the age-associated increases observed both in the intensity and the number of SA- $\beta$ Gal-positive cells in kidney, lung, and liver (Figs. 7g,h). Together, we propose that the reduction in senescence markers is evidence that treatments with GNF 5837 and PF 06273340 are promising strategies to reduce the senescent cells that accumulate with aging. Although GNF 5837 and PF 06273340 do not cross the BBB and thus do not eliminate senescent cells in the central nervous system, this may be an advantageous trait, as it avoids interference with BDNF-TrkB signaling in the brain. 


\section{Discussion}

Strategies to remove detrimental senescent cells from tissues and organs are gaining interest in the clinic, particularly for the treatment of chronic age-related diseases. Given that senescent cells bring about a proinflammatory state through the SASP, they have been found to exacerbate many agingassociated pathologies, including cardiovascular disease, neurodegeneration, cancer, and sarcopenia ${ }^{4,34}$. Therefore, extensive efforts are underway to develop pharmacological approaches to eliminate them with minimum side effects ${ }^{5,35}$. With increasing recognition that MAPK signaling cascades are implicated in senescence ${ }^{9}$, we systematically surveyed the effectiveness of MAPK inhibitors in achieving senolysis. The discovery that Trk inhibitors selectively reduced senescent cell viability (Fig. 1) led us to propose a novel role for TrkB in supporting the survival of senescent cells. Although a role for TrkB in senescence was not previously described, TrkB is a well-established pro-survival receptor in neurons ${ }^{10,11,15}$ and thus we set out to characterize the function of TrkB in senescent cell survival.

Interestingly, the levels of TrkB ligand BDNF, a neurotrophin, were found specifically elevated in several senescence models (Extended Data Fig. 4), and moreover, interfering with BDNF function by silencing or blocking antibodies caused a specific reduction in the viability of senescent cells (Figs. $\mathbf{4 , 5}$ ). Our results indicated that BDNF was required for the pro-survival influence of TrkB, akin to the enhanced survival of neuronal cells elicited by BDNF upon TrkB activation ${ }^{36}$; as in neurons, this regulation appeared to be mediated in senescent cells in an autocrine/paracrine manner. The active secretion of molecules by senescent cells through the SASP is a key feature of the response to damage. SASP factors mount a repair program that includes the promotion of inflammation, recruitment of immune cells, and remodeling of the tissue architecture. The discovery that the SASP factor BDNF promotes senescent cell survival by activating TrkB uncovers an important new dimension of the SASP in strengthening the anti-apoptotic attribute of senescent cells.

The finding that a neuronal survival paradigm is utilized by senescent cells to support viability was interesting, but it was not entirely unexpected, as activation of TrkB by BDNF (and more generally Trk receptors by neurotrophins) has been found to occur outside of the nervous system, particularly in developmental processes associated with the kidney, heart, muscle, and immune system ${ }^{37}$. In addition, the BDNF-TrkB axis has been implicated in tissue repair following lung injury, periodontal wounding, and bone repair ${ }^{28,38,39}$, even though senescence was not studied in such paradigms. On the other hand, aberrant activation of the BDNF-TrkB axis has been described in age-related pathologies such as lung fibrosis, sarcopenia, cancer, and kidney disease $\mathrm{e}^{40-44}$. Interestingly, all these processes, from the developmental responses to the age-associated declines and diseases, have been linked to cellular senescence ${ }^{45-50}$. Thus, it will be important to determine if BDNF contributes to these paradigms through its actions on TrkB-expressing senescent cells. 
Anerillas et al. page 13

In senescent cells, the activation of TrkB by BDNF increased progressively as senescence advanced (Figs. 4, 5). The fact that this signaling paradigm is active in cells facing sublethal levels of DNA damage, but not in cells exposed to higher levels of damage that do not trigger senescence and instead result in cell death (Fig. 6), supports the existence of a well-orchestrated molecular program to efficiently induce senescence within a specific range of injury. In this regard, in cells facing damage that might result in either senescence or death, BDNF appears to be a better predictor of a path to senescence than other, more established, senescence markers such as p21 or IL6, which are also strongly induced by acute DNA damage but are not always good indicators of senescence ${ }^{51-53}$. To test this possibility, a comprehensive analysis of BDNF production in a wide range of senescence models is warranted. Furthermore, given that depleting BDNF levels in cells undergoing senescence drastically reduces viability (Fig. 4, 5), BDNF appears to function not only as a robust marker of cell survival, but also as a key effector of the anti-apoptotic program of senescence. In sum, the activation of BDNFTrkB in senescent cells supports the notion that senescence is a developmental program set in motion by cell damage that shares previously unrecognized molecular similarities with other cell responses such as neuronal survival.

Interestingly, the discovery that the activation of TrkB by BDNF enhanced ERK5 signaling to increase the levels of BCL2L2 had additional parallels with the neuronal responses, as a similar, BCL2L2-dependent program is activated in neurons to persist within the nervous system ${ }^{26}$. Together with the fact that $B C L 2 L 2$ mRNA was identified as a core senescence-associated protein elevated in multiple cellular senescence models ${ }^{25}$, and the fact that the senolytic drug ABT-737, an inhibitor of BCL2L2, potently sensitizes senescent cells to apoptotic death ${ }^{7,8}$, a prominent role for BCL2L2 in senescent cell survival is increasingly apparent. In further support for this notion, we recently found that BCL2L2, but not BCL2L1, contributed to sustaining cell viability in early senescence in a model of senescence that relied on the activation of the oncoprotein SRC (Anerillas et al., submitted).

A major caveat that must be overcome before the BDNF-TrkB paradigm can be exploited therapeutically is the fact that BDNF-TrkB activity is required to maintain a fully functional nervous system $^{54}$. Since Trk inhibition is used to treat chronic pain ${ }^{55}$, various compounds have been developed that prevent adverse side effects within the central nervous system. Two out of the three drugs tested in this work, GNF 5837 and PF 06273340, are peripherally restricted Trk inhibitors, i.e., they cannot cross the blood-brain barrier ${ }^{12,56}$, and thus could be exploited for removing senescent cells through BDNFTrkB inhibition without impacting neuronal function. These compounds reduced senescence-associated markers in tissues such as kidney, lung, and liver in a mouse model of senescence triggered by old age (Fig. 7). Further exploration of the impact of GNF 5837 and PF 06273340 in mice is warranted, particularly in other senescence models, in order to determine if their beneficial actions are similar to those of other senolytics ${ }^{4,34}$. Eventually, it will be essential to study if these or other TrkB inhibitors are viable strategies to reduce the burden of senescent cells in human conditions of dysfunction and disease. 
Anerillas et al. page 14

In summary, TrkB inhibitors reduce senescent cells in aged mouse organs. In sum, we have identified a role for BDNF in promoting cell survival through the activation of TrkB, and propose that this signaling paradigm might be exploited therapeutically to lower senescent cells in older organs. 


\section{Methods}

Cell culture and treatment. Human IMR-90 (ATCC), WI-38 (Coriell Institute), and BJ fibroblasts (ATCC) were cultured in Dulbecco's modified Eagle's medium (DMEM, Gibco) supplemented with $10 \%$ heat-inactivated fetal bovine serum (FBS, Gibco), Penn/Strep (Gibco), Sodium Pyruvate (Gibco), and non-essential amino acids (Gibco) in a $5 \% \mathrm{CO}_{2}$ incubator. Cells were maintained at low population doubling levels (PDL), between PDL15 and PDL25. Etoposide was added to the media as indicated every three days; control conditions included equivalent concentrations of vehicle (DMSO). The remaining drugs utilized were refreshed every 48 hours. The drugs used are listed (Supplementary Table 1).

Cells were transfected with RNAiMAX (Invitrogen) following the manufacturer's instructions. Briefly, cells at 50\% confluency were transfected with ON-TARGETplus SMARTPool (Dharmacon) non-targeting siCtrl (Catalog ID: D-001810-10-05), siNTrk1 (Catalog ID: L-003159-00-0005), siNTrk2 (Catalog ID: L-003160-00-0005), siTP53 (Catalog ID: L-003329-00-0005), siRELA (Catalog ID: L003533-00-0005), siBDNF (Catalog ID: L-017626-00-0005), or siSTAT3 (Catalog ID: L-003544-000005) siRNAs at a final concentration of $25 \mathrm{nM}$. Twenty-four hours later, additional treatments were initiated as indicated, and viability was assessed by direct cell counting and represented as the percentage of remaining cells compared to the number of cells present at the beginning of the experiment. All cell counts were performed manually by using ImageJ and performed in at least three independent replicates. From each replicate, three fields were randomly selected and counted.

RT-qPCR analysis. Samples (tissues or cells) were lysed in either Tri-Reagent (Invitrogen) or RLT buffer (Qiagen), and the lysate was processed with the QIAcube (Qiagen) to purify total RNA, which was then reverse-transcribed (RT) to create cDNA using Maxima reverse transcriptase (Thermo Fisher Scientific) and random hexamers. Real-time, quantitative (q)PCR analysis was then performed using SYBR Green mix (Kapa Biosystems), and the relative expression was determined by the $2^{-\Delta \Delta \mathrm{Ct}}$ method and normalized to $A C T B$ mRNA levels.

The primers used for human transcripts, each forward $(\mathrm{F})$ and reverse $(\mathrm{R})$ were: GTTACGGTCGGAGGCCG and GTGAGAGTGGCGGGGTC for $p 16 / C D K N 2 A$ mRNA; AGTCAGTTCCTTGTGGAGCC and CATGGGTTCTGACGGACAT for $p 21 / C D K N 1 A$ mRNA; AGTGAGGAACAAGCCAGAGC and GTCAGGGGTGGTTATTGCAT for IL6 mRNA; CTTCCAGCCGAGGTCCTT and CCCTGGACACCAACTATTGC for TGFB1 mRNA; GATGGTGGCCTACCTGGAGA and AGAGCTGTGAACTCCGCCCA for BCL2L2 mRNA; GGCTTGACATCATTGGCTGAC and CATTGGGCCGAACTTTCTGGT for BDNF mRNA; GCAAGGCTGATAACGCTGAGGA and CCTGGGCATCAGCGGTCAATG for NTF4 mRNA; CAAGCAGATGGTGGACGTTAAGG and TCGCAGCAGTTCGGTGTCCATT for NTF3 mRNA; 
ACCCGCAACATTACTGTGGACC and GACCTCGAAGTCCAGATCCTGA for NGF mRNA; CCGACACTGTGGTCATTGGCAT and CAGTTCTCGCTTCAGCACGATG for NTRK3 mRNA; TCGTGGCATTTCCGAGATTGG and TCGTCAGTTTGTTTCGGGTAAA for NTRK2 mRNA; CACTAACAGCACATCTGGAGACC and TGAGCACAAGGAGCAGCGTAGA for NTRK1 mRNA; CATGTACGTTGCTATCCAGGC and CTCCTTAATGTCACGCACGAT for ACTB mRNA; GACCTCAACGCACAGTACGAG and AGGAGTCCCATGATGAGATTGT for PUMA mRNA; GAGCTGGTGGTTGACTTTCTC and TCCATCTCCGATTCAGTCCCT for BCL2L1 mRNA; CCTCAGCATCTTATCCGAGTGG and TGGATGGTGGTACAGTCAGAGC for TP53 mRNA; ATGTGGAGATCATTGAGCAGC and CCTGGTCCTGTGTAGCCATT for RELA mRNA; and CAGCAGCTTGACACACGGTA and AAACACCAAAGTGGCATGTGA for STAT3 mRNA.

The primers used for mouse transcripts, each forward $(F)$ and reverse $(R)$ were: CTGCAAGAGACTTCCATCCAG and AGTGGTATAGACAGGTCTGTTGG for $I l 6$ mRNA; GAAATGCCACCTTTTGACAGTG and TGGATGCTCTCATCAGGACAG for $I l l b$ mRNA; CCCAACGCCCCGAACT and GCAGAAGAGCTGCTACGTGAA for $p 16 / C d k n 2 a$ mRNA; TTCTTTGCAGCTCCTTCGTT and ATGGAGGGGAATACAGCCC for Actb mRNA; and TTGCCAGCAGAATAAAAGGTG and TTTGCTCCTGTGCGGAAC for $p 21 / C D K N 1 a$ mRNA.

Caspase 3/7 activity. Apoptosis-related Caspase 3/7 activity was assayed by using the Caspase-Glo 3/7 Assay System (Promega). Briefly, Caspase-Glo® 3/7 solution was added directly to each well. The plate was then shaken vigorously for $30 \mathrm{sec}$ and incubated at $25^{\circ} \mathrm{C}$ in the dark for 30 to $180 \mathrm{~min}$. Luminescence was then measured using a GloMax plate reader (Promega).

Immunofluorescence analysis in cultured cells. Cells were fixed in 4\% PFA (paraformaldehyde) for 10 min and then permeabilized by incubating cells with $0.2 \% \mathrm{TX}-100$ for $5 \mathrm{~min}$ at $25^{\circ} \mathrm{C}$, unless stated otherwise. After blocking with 10\% goat serum for $1 \mathrm{~h}$, primary antibodies were added in the same $10 \%$ goat serum buffer overnight at $4^{\circ} \mathrm{C}$ (phosphorylated STAT3 (Tyr705), 1:50, Cell Signaling 9145; BDNF, 1:40, Santa Cruz Biotechnology sc-65514, TrkB, 1:40, Santa Cruz Biotechnology sc-377218; DPP4, 1:100, Cell Signaling 67138).

Fluorescent signals were detected by adding fluorescent secondary antibodies (Invitrogen, Goat anti-Mouse IgG $(\mathrm{H}+\mathrm{L})$ Cross-Adsorbed Secondary Antibody, Alexa Fluor 568; and Goat anti-Rabbit IgG (H+L), Superclonal Recombinant Secondary Antibody, Alexa Fluor 488) in 10\% goat serum buffer (1:1000) for $1 \mathrm{~h}$ at $25^{\circ} \mathrm{C}$. Finally, DAPI was used to counterstain the nuclei; images were taken using a fluorescence microscope (BZ-X Analyzer, Keyence) and analyzed with ImageJ.

Western blot analysis. Protein extracts were obtained by lysing cells with a denaturing buffer containing 2\% sodium dodecyl sulfate (SDS) (Sigma-Aldrich) in $50 \mathrm{mM}$ HEPES. After boiling and 
Anerillas et al. page 17

sonication, whole-cell protein extracts were separated by size through polyacrylamide gels and transferred to nitrocellulose membranes (Bio-Rad). Membranes were blocked with 5\% non-fat dry milk and immunoblotted. Specific primary antibodies were used that recognized phosphorylated p38 MAPK (T180/Y182) (Biolegend, Ref. 903501), phosphorylated SAPK/JNK (T183/Y185) (81E11) (Cell Signaling, Ref. 4668S), phosphorylated ERK1/2 (T202/Y204) (Biolegend, 675502), phosphorylated AKT (Ser473) (Cell Signaling, 4060S), phosphorylated STAT3 (Y705) (D3A7) XP® (Cell Signaling, 9145S), p53 (DO-1) (Cell Signaling, 18032S), ACTB ( $\beta$-Actin C4) (Santa Cruz Biotechnology, sc47778), and BCL2L2/BCL-w (Cell Signaling, 2724S), phosphorylated PKC $\alpha / \beta$ II (Thr638/641) (Cell Signaling, 9375S), phosphorylated ERK5 (Thr218/Tyr220) (Cell Signaling, 3375), p21 (Santa Cruz Biotechnology, sc-53870), TrkA (Cell Signaling, 2510S), and TrkB (Santa Cruz Biotechnology, sc136990). After incubation with the required secondary antibodies conjugated with horseradish peroxidase (HRP; Jackson Immunoresearch), the chemiluminescent signals were detected by using the Chemidoc system (Biorad).

Isolation of cell surface proteins. Cell surface proteins were isolated by using the Pierce ${ }^{\mathrm{TM}}$ Cell Surface Biotinylation and Isolation Kit (ThermoFisher Scientific, Catalog No. A44390). Briefly, cells were washed twice with PBS and cell surface proteins were labeled for $10 \mathrm{~min}$ at $25^{\circ} \mathrm{C}$ with a solution of EZ-Link Sulfo-NHS-SS-Biotin, a membrane-impermeable biotinylation reagent, followed by several washes in TBS. Cells were then lysed with the detergent provided in the kit and the same amount of protein extract between different samples was used for the subsequent pulldown. The biotinylated proteins were captured by biotin-affinity purification with a NeutrAvidin ${ }^{\mathrm{TM}}$ Agarose Resin (Product No. 29200, ThermoFisher Scientific), followed by several washes with the Wash Buffer. Dithiothreitol (DTT) $(10 \mathrm{mM})$ was used in the elution buffer to reduce the disulfide bonds in the biotin label and release the bound proteins.

Mice. All mouse work, including the import, housing, experimental procedures, and euthanasia, were approved by the Animal Care and Use Committee (ACUC) of National Institute on Aging (NIA). C57BL/6J Mice were provided standard chow ad libitum and maintained under a 12:12 h light/dark cycle. For the delivery of Trk inhibitors, 21-month-old mice were injected intraperitoneally for three months and euthanized at 24 months of age. The treatments were performed at the beginning of each month for 5 consecutive days, at the indicated doses (GNF 5837 at $20 \mathrm{mg} / \mathrm{kg}$, PF $0627334025 \mathrm{mg} / \mathrm{kg}$ ).

Bioplex analysis of mouse serum. Mouse serum was collected, allowed to clot for $2 \mathrm{~h}$ at $25^{\circ} \mathrm{C}$, and centrifuged for $20 \mathrm{~min}$ at $2000 \times \mathrm{g}$. Serum was removed and frozen at $-80^{\circ} \mathrm{C}$. For the multiplex assay, serum was thawed and centrifuged at $16,000 \times \mathrm{g}$ for $4 \mathrm{~min}$. A custom murine Luminex Assay kit was designed by R\&D Biosystems to include the following analytes: GDF-15, PAI-1, and TIMP-1. Serum 
was diluted 1:2 using Calibrator Diluent RD6-52 provided in the kit. Standards (provided with the kit), blanks and serum were incubated with the microparticle cocktail for $2 \mathrm{~h}$ at $25^{\circ} \mathrm{C}$, followed by incubation with a biotin-antibody cocktail for $1 \mathrm{~h}$. A final incubation of $30 \mathrm{~min}$ with Streptavidin-PE was performed with shaking at $25^{\circ} \mathrm{C}$ before running the plate on the Biorad Bioplex-200 Instrument. Each incubation was followed by three sets of washes with wash buffer (provided in the kit). Instrument settings were adjusted to the following: $50 \mu 1$ sample volume, Bio-Plex MagPlex Beads (Magnetic), Double Discriminator Gates set at 8000 and 23,000, low RP1 target value for the CAL2 setting, 50 count/region. The results were analyzed with the Bio-Plex Manager software.

BrdU incorporation. Cells were incubated with $(4 \mu \mathrm{g} / \mathrm{ml})$ 5-Bromo-2'-deoxyuridine (BrdU) diluted in DMEM media with $10 \%$ FBS for $24 \mathrm{~h}$. BrdU incorporation was measured following the manufacturer's protocol (Cell Signaling Technology). Briefly, cells were fixed and denatured prior to the addition of BrdU mouse mAb, and BrdU was detected using the GloMax plate reader (Promega).

Detection of SA- $\boldsymbol{\beta}$-Gal. Senescence-associated $\beta$-Galactosidase (SA- $\beta-G a l)$ activity was assessed following the manufacturer's instructions (Cell Signaling Technology). For the in vivo samples, dried slides were pre-washed with PBS plus $1 \mathrm{mM}$ Magnesium Chloride before the addition of staining solution. All the slides were stained at once for $16 \mathrm{~h}$ at $37^{\circ} \mathrm{C}$. The resulting images were taken by a fluorescence microscope (BZ-X Analyzer, Keyence) and analyzed with ImageJ.

Tissue processing. To perform RT-qPCR analysis in tissue samples, organ pieces were flash-frozen in liquid nitrogen and preserved at $-80^{\circ} \mathrm{C}$ until required. To extract RNA, tissue samples were introduced in Tri-Reagent (Invitrogen) and disrupted using a tissue homogenizer (Bertin Instruments). RNA extraction was then carried out as indicated by the manufacturer's instructions.

For histological analysis, tissue biopsies were immediately fixed in $4 \%$ PFA in PBS at $4{ }^{\circ} \mathrm{C}$ overnight. The following day, tissues were cryoprotected in $30 \%$ sucrose solution in PBS at $4{ }^{\circ} \mathrm{C}$ overnight, and subsequently included in OCT embedding compound, and stored at $-80{ }^{\circ} \mathrm{C}$ until needed. For tissue cutting, OCT blocks were processed using a cryostat at $-20{ }^{\circ} \mathrm{C}$ to obtain $10-\mu \mathrm{m}$ slices that were mounted onto Superfrost ${ }^{\mathrm{TM}}$ Plus Microscope Slides (Fisher Scientific) and dried out overnight at $25^{\circ} \mathrm{C}$. Before incubating with antibodies, antigen retrieval was performed with a sodium citrate-based buffer (Abcam) following the manufacturer's instructions. Tissue slides were then permeabilized with 0.2\% TX-100 in PBS for 10 min, blocked with goat serum blocking solution (Biolegend) for $1 \mathrm{~h}$, and incubated overnight with the antibodies. The antibodies used for immunofluorescence recognized BDNF [EPR1292] (ab108319) (1:100 dilution in blocking solution) and CDKN2A/p16INK4a [2D9A12] (ab54210) (1:250 dilution in blocking solution). Fluorescent secondary antibodies 
(Invitrogen) were added in $10 \%$ goat serum for $1 \mathrm{~h}$ at $25^{\circ} \mathrm{C}$ and slides were mounted with ProLong Diamond Antipode Mountant with DAPI (Life Technologies) and coverslips.

RNA-seq analysis. RNA was extracted with RLT buffer (Qiagen) and purified using the QIAcube system (Qiagen) using RNeasy plus. The quality and quantity of RNA were assessed using the Agilent RNA 6000 nano kit on the Agilent Bioanalyzer. High-quality RNA (125 ng) was used for sequencing the library prep using Illumina TruSeq Stranded mRNA Library prep kit following the manufacturer's protocol (Illumina, San Diego, CA). The quality and quantity of the libraries were checked using the Agilent DNA 1000 Screen Tape on the Agilent Tapestation. Paired-end sequencing was performed for 103 cycles with an Illumina NovaSeq sequencer. BCL files were de-multiplexed and converted to FASTQ files using bcl2fastq program (v2.20.0.422). FASTQ files were trimmed for adapter sequences using Cutadapt version v1.18 and aligned to human genome hg19 Ensembl v82 using STAR software v2.4.0j. featureCounts (v1.6.4) software was used to generate gene counts. The Bioconductor package DESeq2 version $1.30 .0^{57}$ in $\mathrm{R}$ (version 4.0.3) was used to compare counts levels; statistical testing was carried out with Wald test. Transcripts were considered differentially regulated if absolute $\log 2$ fold change was $>1$ and the Benjamini-Hochberg adjusted p-value was $<0.05$. Functional analysis of the differentially expressed genes was performed by using normalized counts in the GSEA platform ${ }^{58}$. RNA-seq data are deposited in GSE190998 (reviewer token crqxgaculzcxnid; https://www.ncbi.nlm.nih.gov/geo/query/acc.cgi?acc=GSE190998).

Statistical Analysis. Data are presented as the means \pm standard deviation (S.D.) of at least $n=3$ independent experiments. Individual data points are displayed in all the bar plots. Significance was established using two-tailed Student's t test $\left(*, \mathrm{p}<0.05,{ }^{* *}, \mathrm{p}<0.01, * * *, \mathrm{p}<0.001\right)$. All analyses were performed with Prism 9. 


\section{Figure Legends}

Fig. 1 | Screening of a library of drugs directed at MAPK signaling proteins unveils Trk inhibitors as potential senolytic compounds. a, Overview of the experimental strategy of the survey. Briefly, senescent and proliferating WI-38 and BJ fibroblasts were treated for $48 \mathrm{~h}$ with a collection of MAPK inhibitors, whereupon cell viability was assessed. b. Schematic representation of the protocol used to reach full senescence using etoposide (Etop, 25 and $50 \mu \mathrm{M}$ for BJ and WI-38 cells, respectively) for 10 days, refreshing etoposide twice, before treating the cell cultures with each of the drugs in the library for an additional $48 \mathrm{~h}$. c, Bar plot displaying the \% change of proliferating and senescent WI-38 fibroblasts (top graph) and BJ fibroblasts (bottom graph) after treatment with the drug library for $48 \mathrm{~h}$ (day 12) relative to the time before drug addition (day 10). Red line, percentages of cells present at each condition right before starting the treatments at day 10. d, Heat map representing the percentage of senolysis (percentage of cell death) after 48 hours when comparing day 12 to day 10 in senescent cells. Drugs were grouped by the target proteins inhibited within the MAPK superfamily. Graphs in c represent the mean values \pm SD of $n=3$ experiments. See also Extended Data Fig. 1.

\section{Fig. 2 | Pharmacological inhibition of TrkB selectively induces apoptotic death of senescent} cells. a, Cell viability curves obtained after testing a range of concentrations of Trk inhibitor drugs (GNF 5837, ANA 12, PF 06273340) for $48 \mathrm{~h}$ in either senescent or proliferating WI-38 fibroblasts. b, Representative micrographs of select doses for each drug tested in (a). Black bar, $100 \mu \mathrm{m}$. c,d, Cell viability (c) and caspase 3/7 (d) activity were assessed $48 \mathrm{~h}$ after treatment as indicated in (b). e, Cell viability assessment in WI-38 cells that had reached replicative senescence (RS) and IR-induced senescence (IRIS), and then were treated for $48 \mathrm{~h}$ with the same doses of Trk inhibitors utilized in (b). f,g, Cell viability and caspase 3/7 activity were assessed in BJ and IMR-90 fibroblasts that had been rendered senescent by exposure to etoposide (Etop, 25 and $50 \mu \mathrm{M}$ for BJ and WI-38 cells, respectively) for 10 days, and then treated for $48 \mathrm{~h}$ with the same doses of Trk inhibitors utilized in (b). h,i, Assessments of cell viability (h) and caspase 3/7 (i) activity measured in cells rendered senescent by etoposide for 10 days, then treated for $48 \mathrm{~h}$ with different Trk inhibitors, either alone or in combination with the pan-caspase inhibitor Z-VAD-FMK. Graphs in a, $\mathbf{c}-\mathbf{i}$, represent the mean values \pm SD of $n=3$ experiments; significance $(* \mathrm{p}<0.05, * * \mathrm{p}<0.01$, $* * * \mathrm{p}<0.001)$ was determined by using two-tailed Student's t-test. See also Extended Data Fig. 2.

Fig. 3 | Increased TrkB levels in senescent cells promotes resistance to apoptosis. a, RT-qPCR analysis of the levels of NTRK1, NTRK2, and NTRK3 mRNAs (encoding Trk receptors) and senescence markers p16 mRNA and IL6 mRNA in WI-38 cells rendered senescent by treatment with etoposide (Etop, $50 \mu \mathrm{M}$ ) for 10 days. Data were normalized to the levels of GAPDH mRNA. b, Representative 
Anerillas et al. page 21

western blot analysis of the levels of TrkA, TrkB, senescence marker p21, and loading control ACTB, in WI-38, BJ, and IMR-90 fibroblasts that were proliferating $(\mathrm{P})$ or were rendered senescent $(\mathrm{S})$ by etoposide treatment for 10 days. WI-38 cells rendered senescent by replicative exhaustion (RS) were included in this analysis. c, Cells were transfected with control siRNA (siCtrl) or with siRNAs directed at TrkA and TrkB (siNTrk1 and siNTrk2, respectively), and the percentage of live cells were counted at the indicated times ( $0-10$ days) after treatment with a dose of etoposide $(50 \mu \mathrm{M})$ that triggered senescence. d, RT-qPCR analysis of NTRK1 and NTRK2 mRNA levels after ten days in the presence of with $50 \mu \mathrm{M}$ etoposide, to assess the efficacy of the siRNA interventions. Data were normalized to the levels of GAPDH mRNA. e, Representative western blot analysis of the levels of TrkB, labile protein p53, and loading control ACTB in proliferating and senescent cells at the times shown after incubating the cells with CHX $(50 \mu \mathrm{g} / \mathrm{ml})$. f, Representative western blot analysis of the levels of TrkB, senescence marker p21, and loading control ACTB. g, RT-qPCR analysis of the levels of TGFB1 mRNA (an early senescence marker), p16 mRNA (a late senescence marker), and IL6 mRNA (a late senescence marker). Data were normalized to the levels of GAPDH mRNA. h, Pulldown of cellsurface proteins from WI-38 cells that were either proliferating $(\mathrm{P})$ or rendered senescent $(\mathrm{S})$ by etoposide treatment (50 $\mu \mathrm{M}, 10$ days). The presence of TrkB in the cell-surface pulldown and DPP4, a known senescent cell surface marker, was assessed by western blot analysis. Ponceau staining of the transferred samples served to assess differences in sample loading and transfer. i, Immunofluorescence micrographs to identify cells positive for TrkB (red), DPP4 (green), and merged signals (orange) in proliferating and senescent cells (etoposide $50 \mu \mathrm{M}, 10 \mathrm{~d}$ ) in non-permeabilizing conditions. White scale bar, $100 \mu \mathrm{m}$. Graphs (a,c,d,g) display the mean values \pm SD of $n=3$ experiments; significance $(* \mathrm{p}$ $<0.05, * * \mathrm{p}<0.01, * * * \mathrm{p}<0.001)$ was determined by using two-tailed Student's t-test. See also

\section{Extended Data Fig. 3.}

Fig. 4 | BDNF is a novel SASP member that ensures viability in senescent cells. a, Heat map display of the relative abundance of mRNAs encoding the different neurotrophins in both proliferating and senescent WI-38, BJ, or IMR-90 fibroblasts (50, 25, and $50 \mu \mathrm{M}$, respectively, 10 days), as assessed by RT-qPCR analysis. b, ELISA measurement of BDNF levels in conditioned media collected for $48 \mathrm{~h}$ in the same experimental groups shown in (a). c, Cytokine array analysis of the abundance of several SASP factors, including neurotrophins BDNF, NTF3, and NTF4, in proliferating and senescent (Etop, $50 \mu \mathrm{M}, 10$ days) WI-38 cells. d, Immunofluorescent micrographs of BDNF signals in WI-38 cells processed as in (c). Scale bar, $100 \mu \mathrm{m}$. e, RT-qPCR analysis of BDNF mRNA levels at the times shown as WI-38 cells progress towards senescence. Data were normalized to GAPDH mRNA levels. f, RT-qPCR analysis of the levels of BDNF, p21, IL6, and p16 mRNAs (as well as the levels of TP53 and RELA mRNAs, to monitor the silencing interventions) in senescent cells transfected with either control siRNA ( $\mathrm{siCtrl}$ ) or siRNAs that specifically lowered the abundance of p53 or RELA. Proliferating cells 
Anerillas et al. page 22

transfected with siCtrl were utilized as a control for the baseline expression of each of the mRNAs analyzed. g, Percentage of WI-38 fibroblasts relative to the starting cell numbers after transfecting them with siCtrl or siBDNF and then inducing senescence (50 $\mu \mathrm{M}$ Etop). Cell numbers were measured by direct cell counting at each of time point. h, RT-qPCR analysis of BDNF mRNA levels in the cells studied in (g) at day 10. i, Number of remaining cells after $48 \mathrm{~h}$ of treatment with either IgG or BDNFblocking antibodies ( $4 \mu \mathrm{g} / \mathrm{ml}$ each) in P and S WI-38 fibroblasts treated as in (g), represented as the percentage of live cells relative to initial cell counts. $\mathbf{j}$, Representative micrograph of the experiments plotted in (i), only for S cells. Scale bar, $100 \mu \mathrm{m}$. k, Caspase 3/7 activity analysis performed in senescent WI-38 fibroblasts treated for $48 \mathrm{~h}$ with either IgG or BDNF-blocking antibodies as described in (i). Graphs $(\mathbf{b}, \mathbf{e}-\mathbf{i}, \mathbf{k})$ represent the mean values $\pm \mathrm{SD}$ of $\mathrm{n}=3$ experiments; significance $(* \mathrm{p}<0.05$, **p $\left.<0.01,{ }^{* *} \mathrm{p}<0.001\right)$ was determined by using two-tailed Student's t-test. See also Extended Data Fig. 4.

Fig. 5 | ERK5 activation by TrkB-BDNF axis sustains senescent cell survival through BCL2L2. a, Western blot analysis performed with PhosTag gels to assess TrkB phosphorylation across the senescence time course (left), as well as in cells transfected to express normal (siCtrl) or reduced (siBDNF) levels of BDNF (right). ACTB was used as a loading control. b, WI-38 fibroblasts were transfected with siCtrl, siNTrk2, and siBDNF and treated with $50 \mu \mathrm{M}$ etoposide (Etop) the next day to induce senescence. Cell viability was then measured by direct cell counting at early senescence ( 2 days after adding etoposide) and late senescence ( 8 days after adding etoposide). c, Schematic of the experimental procedure followed for RNA-seq analysis to evaluate transcriptomic differences among WI-38 fibroblasts transfected with siCtrl, siNTrk2, and siBDNF. d, Transcriptomic analysis of WI-38 cells in which NTrk2 or BDNF were silenced. Shown are GSEA gene set associations with the stated conditions (left), and a heat map depicting genes related to apoptosis that changed significantly in senescent cells after silencing TrkB as well as in senescent cells after silencing BDNF (right). e, RTqPCR analysis of the levels of PUMA, BCL2L2, and BCL2L1 mRNAs in early or late senescent WI-38 cells transfected with siCtrl, siNTrk2 or siBDNF, as defined in (b). f, RT-qPCR analysis of the levels of PUMA, BCL2L2, and BCL2L1 mRNAs in proliferating and senescent cells treated with either IgG or BDNF-blocking ( $4 \mu \mathrm{g} / \mathrm{ml}$ each) antibodies for $48 \mathrm{~h}$. g, Representative western blot analysis of the levels of BCLW (BCL2L2), p53, and loading control ACTB in cells treated as described in (e,f). h, Representative western blot analysis of known effector proteins downstream of BDNF-TrkB in other cellular systems, including p-AKT (S473), p-ERK1/2 (T202/Y204), p-p38 (T180/Y182), p-JNK (T183/Y185), p-STAT3 (Y705), p-PKC $\alpha / \beta$ II (T638/641), and p-ERK5 (T218/Y220). Proteins were assessed in cells that were processed as explained in (e,f). i, RT-qPCR analysis of the levels of BCL2L2, PUMA, and BCL2L1 mRNAs in senescent WI-38 cells (50 $\mu \mathrm{M}$ etoposide, 10 days) that were either left untreated or treated with an ERK5 inhibitor (ERK5i, ERK5-IN-1) for 48 h at $1 \mu \mathrm{M}$. j, WI-38 
fibroblasts were treated with the ERK5i as described in (i), and cell viability was measured by direct cell counting of the remaining viable cells. Graphs $(\mathbf{b}, \mathbf{e}, \mathbf{f}, \mathbf{i}, \mathbf{j})$, represent the values \pm SD from $n=3$ experiments; significance $(* \mathrm{p}<0.05, * * \mathrm{p}<0.01, * * * \mathrm{p}<0.001)$ was determined by using two-tailed Student's t-test. See also Extended Data Fig. 5.

Fig. 6 | BDNF is a marker of surviving senescent cells. a,b, WI-38 fibroblasts were treated with the doses of etoposide (Etop) shown; $48 \mathrm{~h}$ later, cell viability was assessed by direct cell counts and represented as percentages referred to initial counts (the red dashed line) (a), and RT-qPCR analysis was used to measure the levels of BDNF, p21, and IL6 mRNAs (normalized to GAPDH mRNA) (b). c, Assessment of $B D N F$ mRNA levels in WI-38 cells treated with etoposide $50 \mu \mathrm{M}$ for $48 \mathrm{~h}$ after silencing p53 (siTP53; siCtrl in control transfections) or inducing p53 levels (by treatment with Nutlin3a, N3A, $10 \mu \mathrm{M}$ ). Controls included siCtrl-transfected and untreated WI-38 cells, respectively. d, RTqPCR analysis of BDNF mRNA levels in WI-38 cells transfected with either siCtrl or siSTAT3 siRNAs and treated for $48 \mathrm{~h}$ with $50 \mu \mathrm{M}$ etoposide. e, Representative western blot analysis of p-STAT3 (Y705) and p53 protein levels in WI-38 cells treated as explained in (c). f, Representative western blot analysis of p-STAT3 (Y705) and p53 protein levels in the experimental groups shown in (a). g,h, Representative immunofluorescence analysis of colocalized signals for p-STAT3 (Y705) (green) and BDNF (red). DAPI was used to stain nuclei. Co-staining for p-STAT3 and BDNF appears as an orange signal (orange arrows). Red arrows point to BDNF-only positive cells. White scale bar, $100 \mu \mathrm{m}$. Signals from the experiment in $(\mathbf{g})$ were quantified $(\mathbf{h})$. Fractions in color represent the percentage $\pm \mathrm{SD}$ of the resulting staining for each group described: unstained, BDNF+, p-STAT3/BDNF+, and p-STAT3+. i , Schematic depicting the proposed model described in this study. Values shown in a-d are the means $\pm \mathrm{SD}$; significance $(* \mathrm{p}<0.05, * * \mathrm{p}<0.01, * * * \mathrm{p}<0.001)$ was determined by using two-tailed Student's t-test. See also Extended Data Fig. 6.

\section{Fig. 7 | Treatment with TrkB inhibitors reduces senescent cells in organs from old mice. a,} Immunofluorescence staining of BDNF (green) and p16 (red) in lung and liver from 24-month old (m.o.) C57BL/6J mice. b, Schedule of administration of TrkB inhibitors GNF 5837 and PF 06273340 to normally aging mice. c, RT-qPCR analysis of the abundance of $p 16, p 21, I l 6$, and $I l 1 b$ mRNAs in kidney, lung and liver in the different mouse cohorts studied. The levels of these mRNAs were normalized to Actb mRNA levels. d, GDF15 concentration in sera from the four mouse cohorts, as measured with a Bioplex instrument. e,f, Percentages of p16-positive cells in kidney, lung, and liver of the four mouse cohorts (e); representative images of p16-positive cells found in kidney in the different groups tested (f). $\mathbf{g}, \mathbf{h}$, Representative images (g) and quantification (h) of SA- $\beta$-Gal activity assessment performed in the same four mouse cohorts described in (c). Scale bars, $200 \mu \mathrm{m}$. Values shown in c-e,h 
Anerillas et al. page 24

are individual data points with the values $\pm \mathrm{SD}$; significance $\left({ }^{*} \mathrm{p}<0.05, * * \mathrm{p}<0.01,{ }^{* * *} \mathrm{p}<0.001\right)$ was determined by using two-tailed Student's t-test. See also Extended Data Fig. 7. 


\section{References}

1 Gorgoulis, V. et al. Cellular Senescence: Defining a Path Forward. Cell 179, 813-827, doi:10.1016/j.cell.2019.10.005 (2019).

2 Birch, J. \& Gil, J. Senescence and the SASP: many therapeutic avenues. Genes Dev. 34, 1565-1576, doi:10.1101/gad.343129.120 (2020).

3 Herranz, N. \& Gil, J. Mechanisms and functions of cellular senescence. J. Clin. Invest. 128, 1238-1246, doi:10.1172/JCI95148 (2018).

4 Di Micco, R., Krizhanovsky, V., Baker, D. \& d'Adda di Fagagna, F. Cellular senescence in ageing: from mechanisms to therapeutic opportunities. Nat. Rev. Mol. Cell. Biol. 22, 75-95, doi:10.1038/s41580-020-00314-w (2021).

5 van Deursen, J. M. Senolytic therapies for healthy longevity. Science 364, 636-637, doi:10.1126/science.aaw1299 (2019).

6 Baar, M. P. et al. Targeted Apoptosis of Senescent Cells Restores Tissue Homeostasis in Response to Chemotoxicity and Aging. Cell 169, 132-147 e116, doi:10.1016/j.cell.2017.02.031 (2017).

7 Chang, J. et al. Clearance of senescent cells by ABT263 rejuvenates aged hematopoietic stem cells in mice. Nat. Med. 22, 78-83, doi:10.1038/nm.4010 (2016).

8 Yosef, R. et al. Directed elimination of senescent cells by inhibition of BCL-W and BCLXL. Nat. Commun. 7, 11190, doi:10.1038/ncomms11190 (2016).

9 Anerillas, C., Abdelmohsen, K. \& Gorospe, M. Regulation of senescence traits by MAPKs. Geroscience 42, 397-408, doi:10.1007/s11357-020-00183-3 (2020).

10 Chao, M. V. Neurotrophins and their receptors: a convergence point for many signalling pathways. Nat. Rev. Neurosci. 4, 299-309, doi:10.1038/nrn1078 (2003).

11 Mitre, M., Mariga, A. \& Chao, M. V. Neurotrophin signalling: novel insights into mechanisms and pathophysiology. Clin. Sci. (Lond) 131, 13-23, doi:10.1042/CS20160044 (2017). 12 Skerratt, S. E. et al. The Discovery of a Potent, Selective, and Peripherally Restricted PanTrk Inhibitor (PF-06273340) for the Treatment of Pain. J. Med. Chem. 59, 10084-10099, doi:10.1021/acs.jmedchem.6b00850 (2016).

13 Cazorla, M. et al. Identification of a low-molecular weight TrkB antagonist with anxiolytic and antidepressant activity in mice. J. Clin. Invest. 121, 1846-1857, doi:10.1172/JCI43992 (2011). 14 Munk, R. et al. Acid ceramidase promotes senescent cell survival. Aging (Albany NY) 13, 15750-15769, doi:10.18632/aging.203170 (2021).

15 Haddad, Y., Adam, V. \& Heger, Z. Trk Receptors and Neurotrophin Cross-Interactions: New Perspectives Toward Manipulating Therapeutic Side-Effects. Front. Mol. Neurosci. 10, 130, doi:10.3389/fnmol.2017.00130 (2017).

16 Munoz-Espin, D. \& Serrano, M. Cellular senescence: from physiology to pathology. Nat. Rev. Mol. Cell. Biol. 15, 482-496, doi:10.1038/nrm3823 (2014).

17 Zhang, Y., Xiong, Y. \& Yarbrough, W. G. ARF promotes MDM2 degradation and stabilizes p53: ARF-INK4a locus deletion impairs both the Rb and p53 tumor suppression pathways. Cell 92, 725-734, doi:10.1016/s0092-8674(00)81401-4 (1998).

18 Hoare, M. et al. NOTCH1 mediates a switch between two distinct secretomes during senescence. Nat. Cell Biol. 18, 979-992, doi:10.1038/ncb3397 (2016).

19 Ito, Y., Hoare, M. \& Narita, M. Spatial and Temporal Control of Senescence. Trends Cell Biol. 27, 820-832, doi:10.1016/j.tcb.2017.07.004 (2017).

20 Purvis, J. E. et al. p53 dynamics control cell fate. Science 336, 1440-1444, doi:10.1126/science.1218351 (2012).

21 Kim, K. M. et al. Identification of senescent cell surface targetable protein DPP4. Genes Dev. 31, 1529-1534, doi:10.1101/gad.302570.117 (2017).

22 Casella, G. et al. Transcriptome signature of cellular senescence. Nucleic Acids Res. 47, 7294-7305, doi:10.1093/nar/gkz555 (2019). 
23 Lipsky, R. H. \& Marini, A. M. Brain-derived neurotrophic factor in neuronal survival and behavior-related plasticity. Ann. N. Y. Acad. Sci. 1122, 130-143, doi:10.1196/annals.1403.009 (2007).

24 Lee, S. \& Schmitt, C. A. The dynamic nature of senescence in cancer. Nat. Cell Biol. 21, 94-101, doi:10.1038/s41556-018-0249-2 (2019).

25 Hernandez-Segura, A. et al. Unmasking Transcriptional Heterogeneity in Senescent Cells. Curr. Biol. 27, 2652-2660 e2654, doi:10.1016/j.cub.2017.07.033 (2017).

26 Hartman, M. L. \& Czyz, M. BCL-w: apoptotic and non-apoptotic role in health and disease. Cell Death Dis. 11, 260, doi:10.1038/s41419-020-2417-0 (2020).

27 Efeyan, A. et al. Induction of p53-dependent senescence by the MDM2 antagonist nutlin-3a in mouse cells of fibroblast origin. Cancer Res. 67, 7350-7357, doi:10.1158/0008-5472.CAN-070200 (2007).

28 Paris, A. J. et al. STAT3-BDNF-TrkB signalling promotes alveolar epithelial regeneration after lung injury. Nat. Cell Biol. 22, 1197-1210, doi:10.1038/s41556-020-0569-x (2020).

29 Baker, D. J. et al. Naturally occurring p16(Ink4a)-positive cells shorten healthy lifespan. Nature 530, 184-189, doi:10.1038/nature16932 (2016).

30 Idda, M. L. et al. Survey of senescent cell markers with age in human tissues. Aging (Albany NY) 12, 4052-4066, doi:10.18632/aging.102903 (2020).

31 Ovadya, Y. et al. Impaired immune surveillance accelerates accumulation of senescent cells and aging. Nat. Commun. 9, 5435, doi:10.1038/s41467-018-07825-3 (2018).

32 Yousefzadeh, M. J. et al. Tissue specificity of senescent cell accumulation during physiologic and accelerated aging of mice. Aging Cell 19, e13094, doi:10.1111/acel.13094 (2020).

33 Basisty, N. et al. A proteomic atlas of senescence-associated secretomes for aging biomarker development. PLoS Biol. 18, e3000599, doi:10.1371/journal.pbio.3000599 (2020).

$34 \mathrm{Xu}, \mathrm{M}$. et al. Senolytics improve physical function and increase lifespan in old age. Nat. Med. 24, 1246-1256, doi:10.1038/s41591-018-0092-9 (2018).

35 Roy, A. L. et al. A Blueprint for Characterizing Senescence. Cell 183, 1143-1146, doi:10.1016/j.cell.2020.10.032 (2020).

36 Vilar, M. \& Mira, H. Regulation of Neurogenesis by Neurotrophins during Adulthood: Expected and Unexpected Roles. Front. Neurosci. 10, 26, doi:10.3389/fnins.2016.00026 (2016). 37 Thiele, C. J., Li, Z. \& McKee, A. E. On Trk--the TrkB signal transduction pathway is an increasingly important target in cancer biology. Clin. Cancer Res. 15, 5962-5967, doi:10.1158/1078-0432.CCR-08-0651 (2009).

38 Matsuda, S. et al. Brain-derived neurotrophic factor induces migration of endothelial cells through a TrkB-ERK-integrin alphaVbeta3-FAK cascade. J. Cell Physiol. 227, 2123-2129, doi:10.1002/jcp.22942 (2012).

39 Kilian, O. et al. BDNF and its TrkB receptor in human fracture healing. Ann. Anat. 196, 286-295, doi:10.1016/j.aanat.2014.06.001 (2014).

40 Avcuoglu, S. et al. Neurotrophic tyrosine kinase receptor B/neurotrophin 4 signaling axis is perturbed in clinical and experimental pulmonary fibrosis. Am. J. Respir. Cell. Mol. Biol. 45, 768780, doi:10.1165/rcmb.2010-01950C (2011).

41 Kalinkovich, A. \& Livshits, G. Sarcopenia--The search for emerging biomarkers. Ageing Res. Rev. 22, 58-71, doi:10.1016/j.arr.2015.05.001 (2015).

42 Cherubini, E. et al. BDNF/TrkB axis activation promotes epithelial-mesenchymal transition in idiopathic pulmonary fibrosis. J. Transl. Med. 15, 196, doi:10.1186/s12967-017-1298-1 (2017). 43 Radin, D. P. \& Patel, P. BDNF: An Oncogene or Tumor Suppressor? Anticancer Res. 37, 3983-3990, doi:10.21873/anticanres.11783 (2017).

44 Endlich, N. et al. BDNF: mRNA expression in urine cells of patients with chronic kidney disease and its role in kidney function. J. Cell. Mol. Med. 22, 5265-5277, doi:10.1111/jcmm.13762 (2018). 
45 Childs, B. G., Durik, M., Baker, D. J. \& van Deursen, J. M. Cellular senescence in aging and age-related disease: from mechanisms to therapy. Nat. Med. 21, 1424-1435, doi:10.1038/nm.4000 (2015).

46 Demaria, M. et al. An essential role for senescent cells in optimal wound healing through secretion of PDGF-AA. Dev Cell 31, 722-733, doi:10.1016/j.devcel.2014.11.012 (2014).

47 Majewska, J. \& Krizhanovsky, V. Breathe it in - Spotlight on senescence and regeneration in the lung. Mech. Ageing Dev. 199, 111550, doi:10.1016/j.mad.2021.111550 (2021).

48 Munoz-Espin, D. et al. Programmed cell senescence during mammalian embryonic development. Cell 155, 1104-1118, doi:10.1016/j.cell.2013.10.019 (2013).

49 Schafer, M. J. et al. Cellular senescence mediates fibrotic pulmonary disease. Nat. Commun. 8, 14532, doi:10.1038/ncomms14532 (2017).

50 Storer, M. et al. Senescence is a developmental mechanism that contributes to embryonic growth and patterning. Cell 155, 1119-1130, doi:10.1016/j.cell.2013.10.041 (2013).

51 Biton, S. \& Ashkenazi, A. NEMO and RIP1 control cell fate in response to extensive DNA damage via TNF-alpha feedforward signaling. Cell 145, 92-103, doi:10.1016/j.cell.2011.02.023 (2011).

52 Karimian, A., Ahmadi, Y.\& Yousefi, B. Multiple functions of p21 in cell cycle, apoptosis and transcriptional regulation after DNA damage. DNA Repair (Amst) 42, 63-71, doi:10.1016/j.dnarep.2016.04.008 (2016).

53 Rodier, F. et al. Persistent DNA damage signalling triggers senescence-associated inflammatory cytokine secretion. Nat. Cell Biol. 11, 973-979, doi:10.1038/ncb1909 (2009).

54 Yoshii, A. \& Constantine-Paton, M. Postsynaptic BDNF-TrkB signaling in synapse maturation, plasticity, and disease. Dev. Neurobiol. 70, 304-322, doi:10.1002/dneu.20765 (2010). 55 Wang, T., Yu, D. \& Lamb, M. L. Trk kinase inhibitors as new treatments for cancer and pain. Expert Opin. Ther. Pat. 19, 305-319, doi:10.1517/13543770902721261 (2009).

56 Albaugh, P. et al. Discovery of GNF-5837, a Selective TRK Inhibitor with Efficacy in Rodent Cancer Tumor Models. ACS Med. Chem. Lett. 3, 140-145, doi:10.1021/ml200261d (2012). 57 Love, M. I., Huber, W. \& Anders, S. Moderated estimation of fold change and dispersion for RNA-seq data with DESeq2. Genome Biol. 15, 550, doi:10.1186/s13059-014-0550-8 (2014). 58 Subramanian, A. et al. Gene set enrichment analysis: a knowledge-based approach for interpreting genome-wide expression profiles. Proc. Natl. Acad. Sci. U. S. A. 102, 15545-15550, doi:10.1073/pnas.0506580102 (2005). 


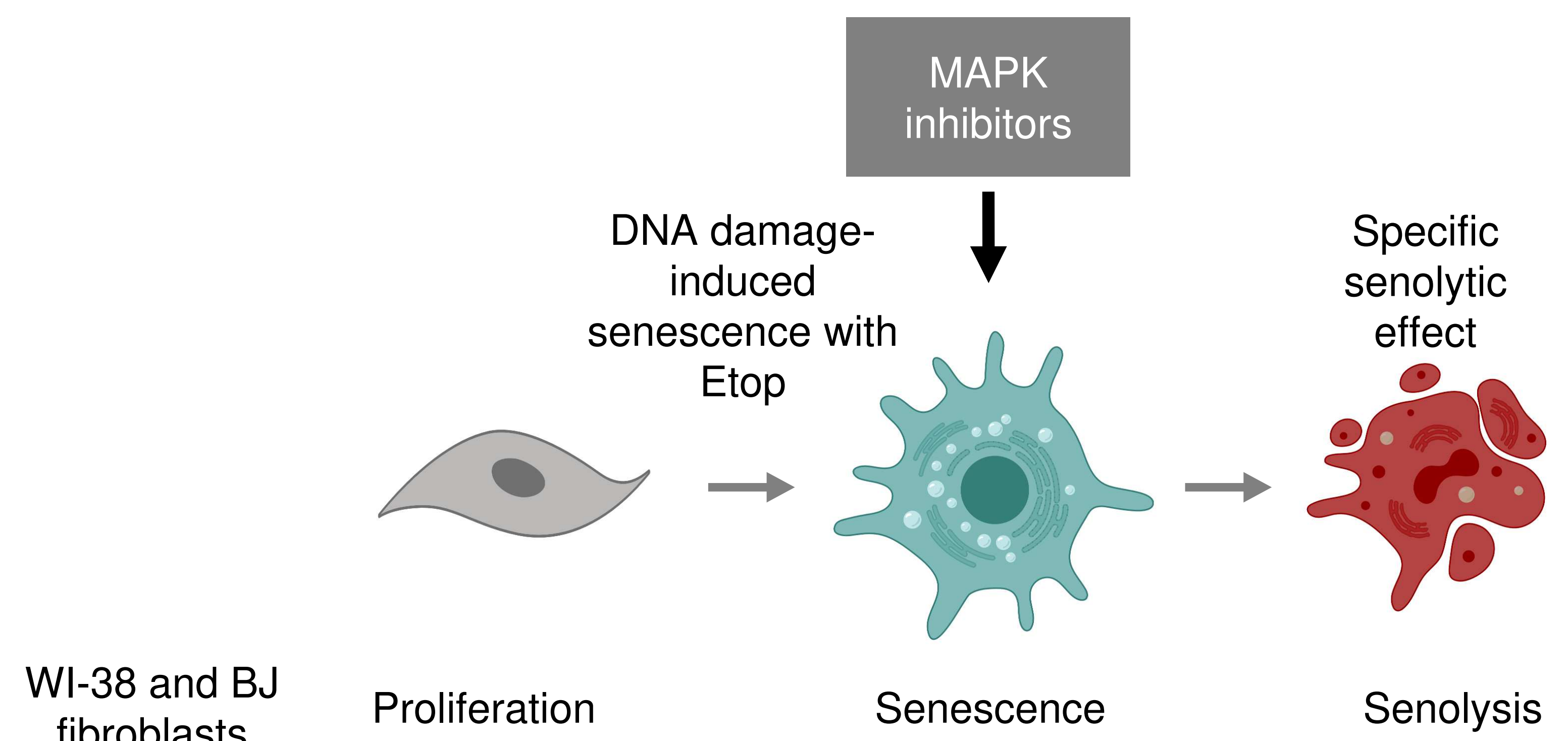

C
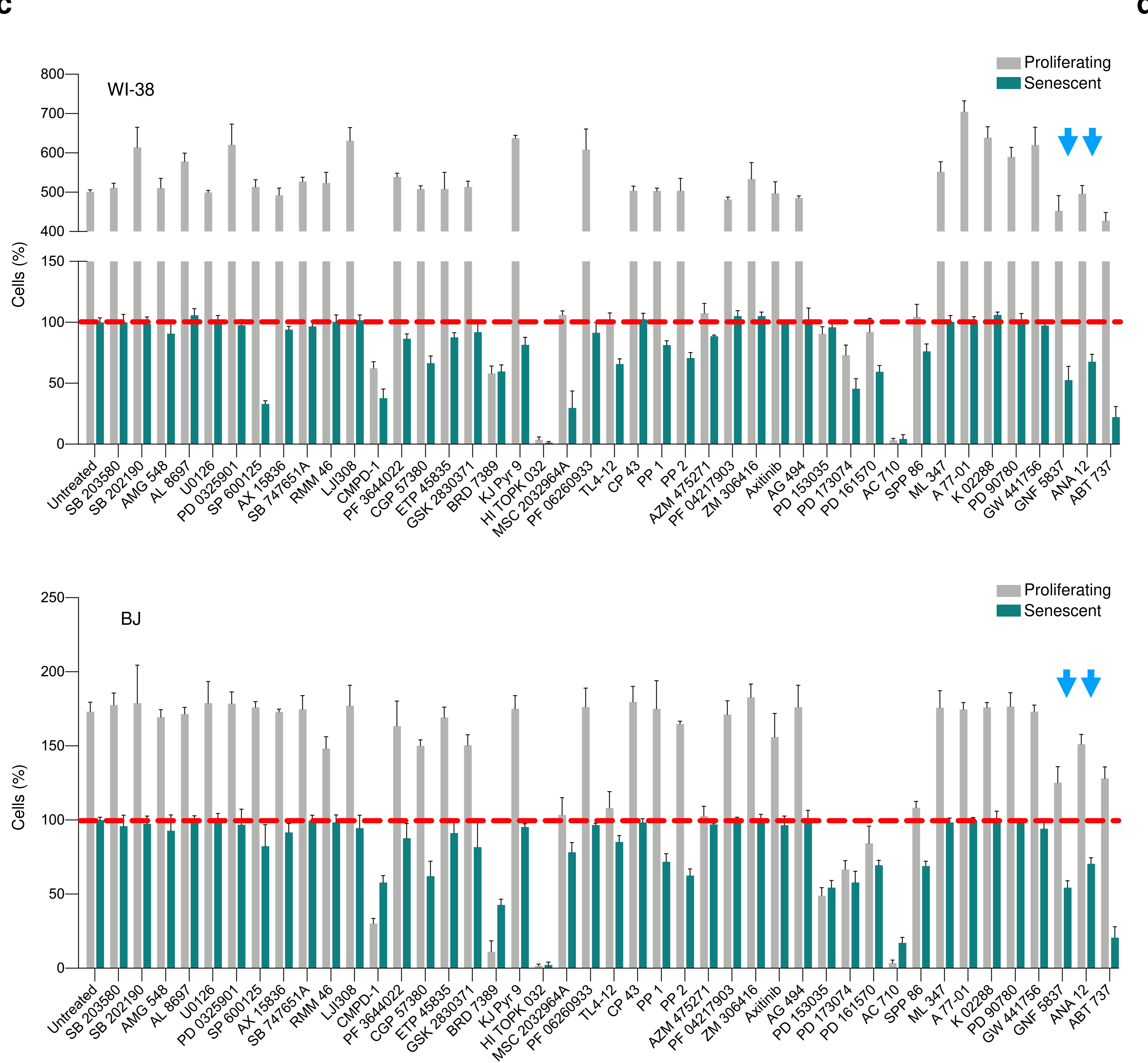

fibroblasts

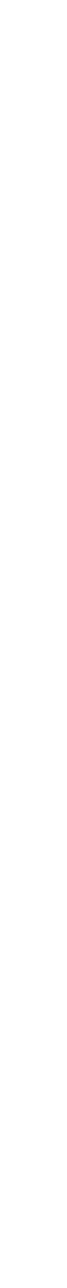

b

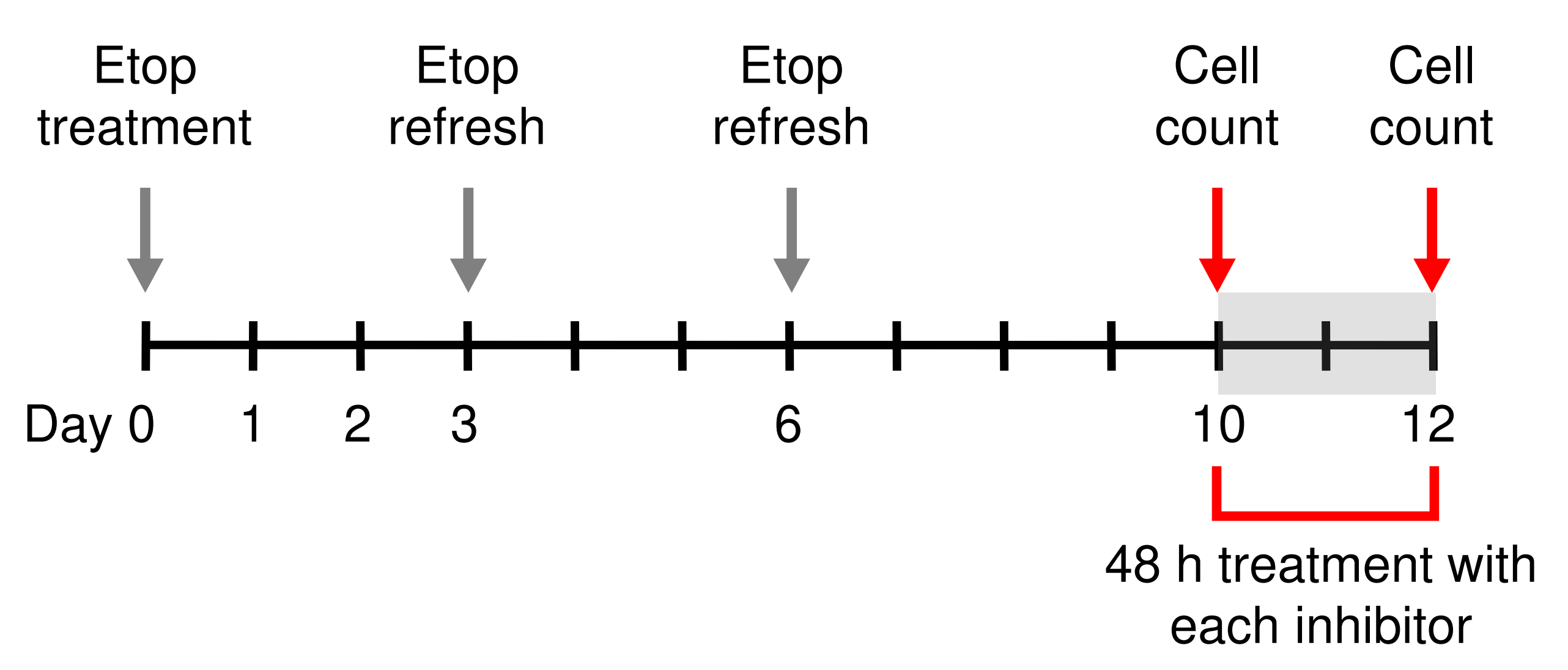


a
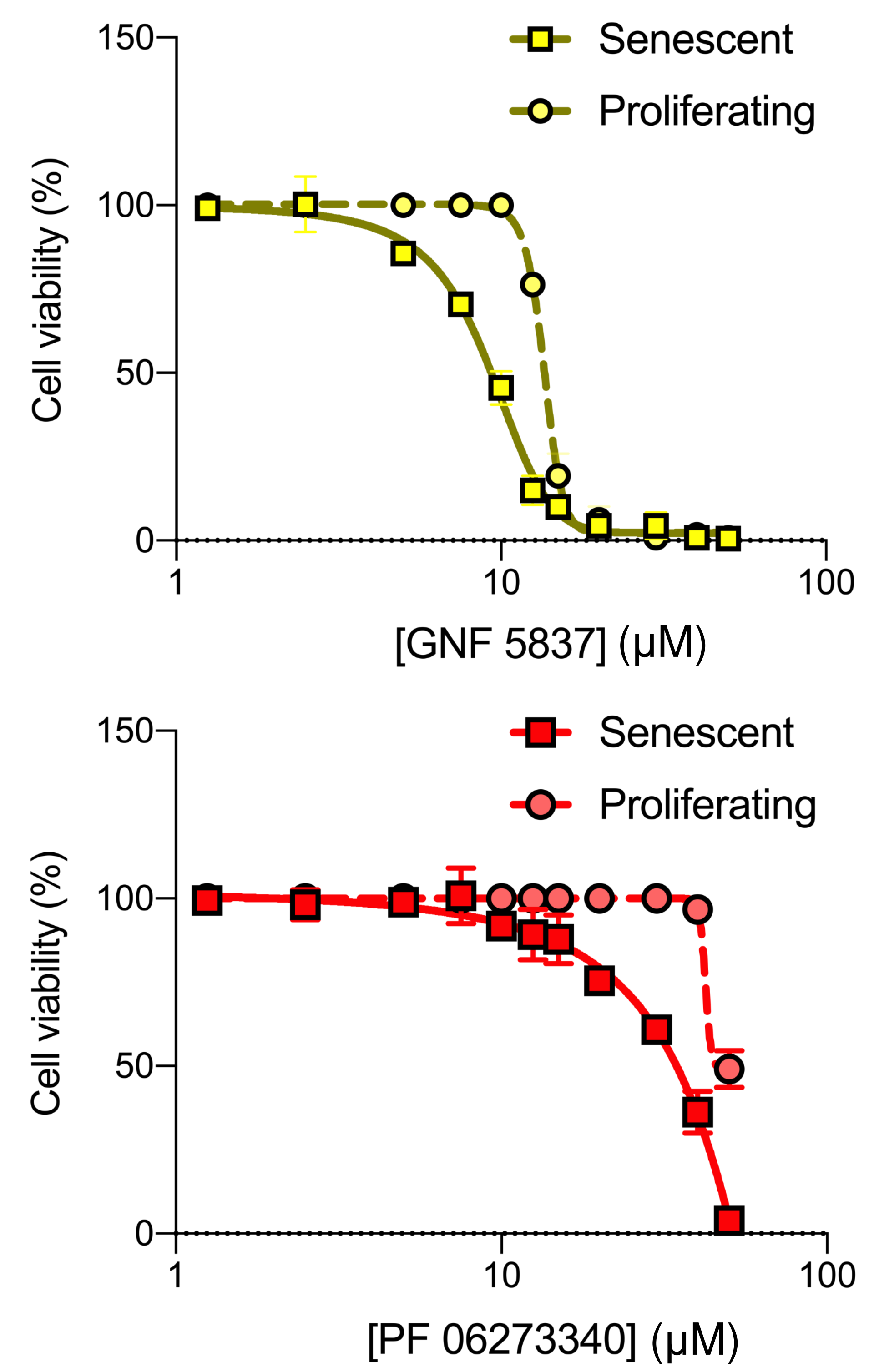

b

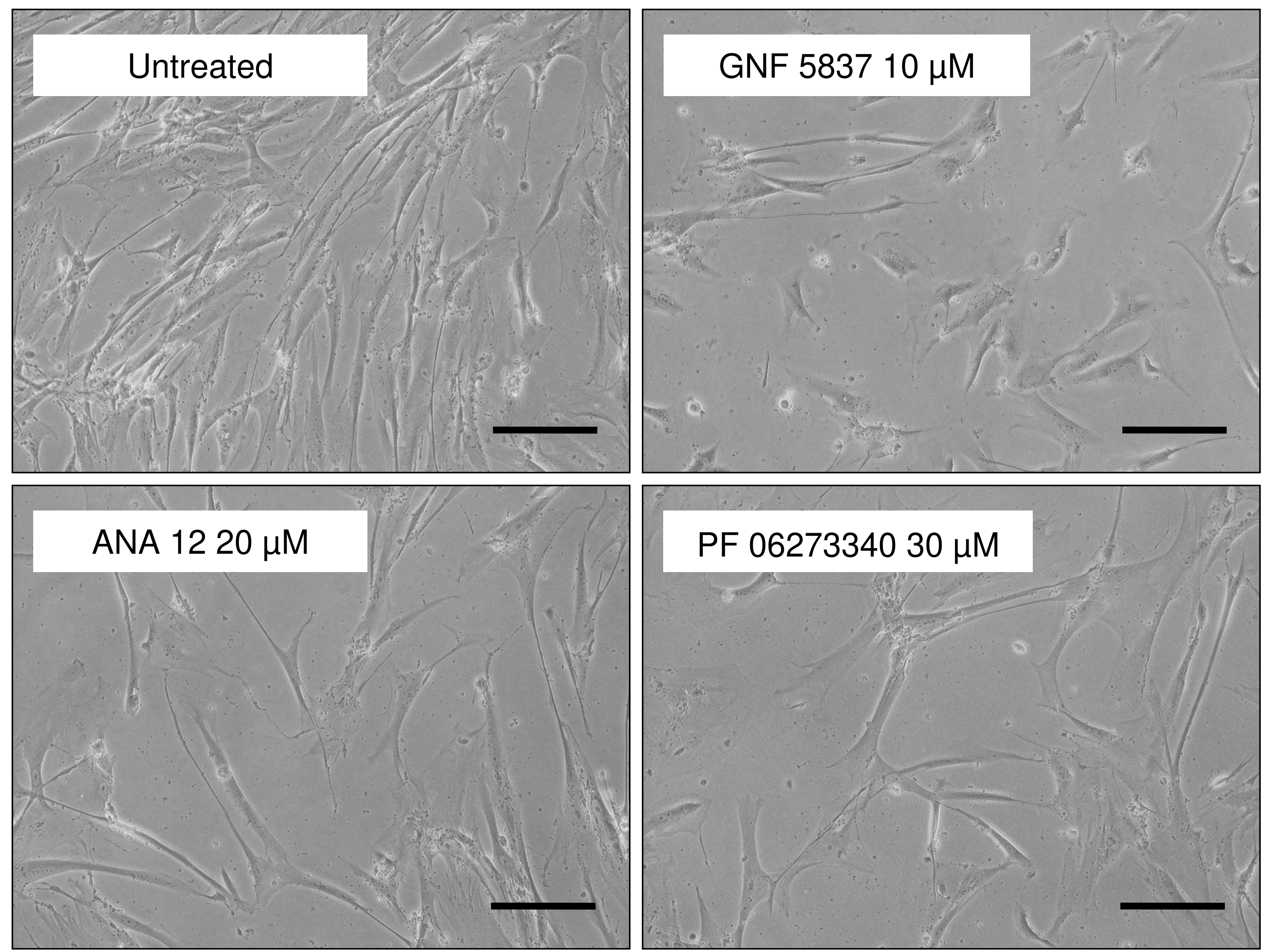

C

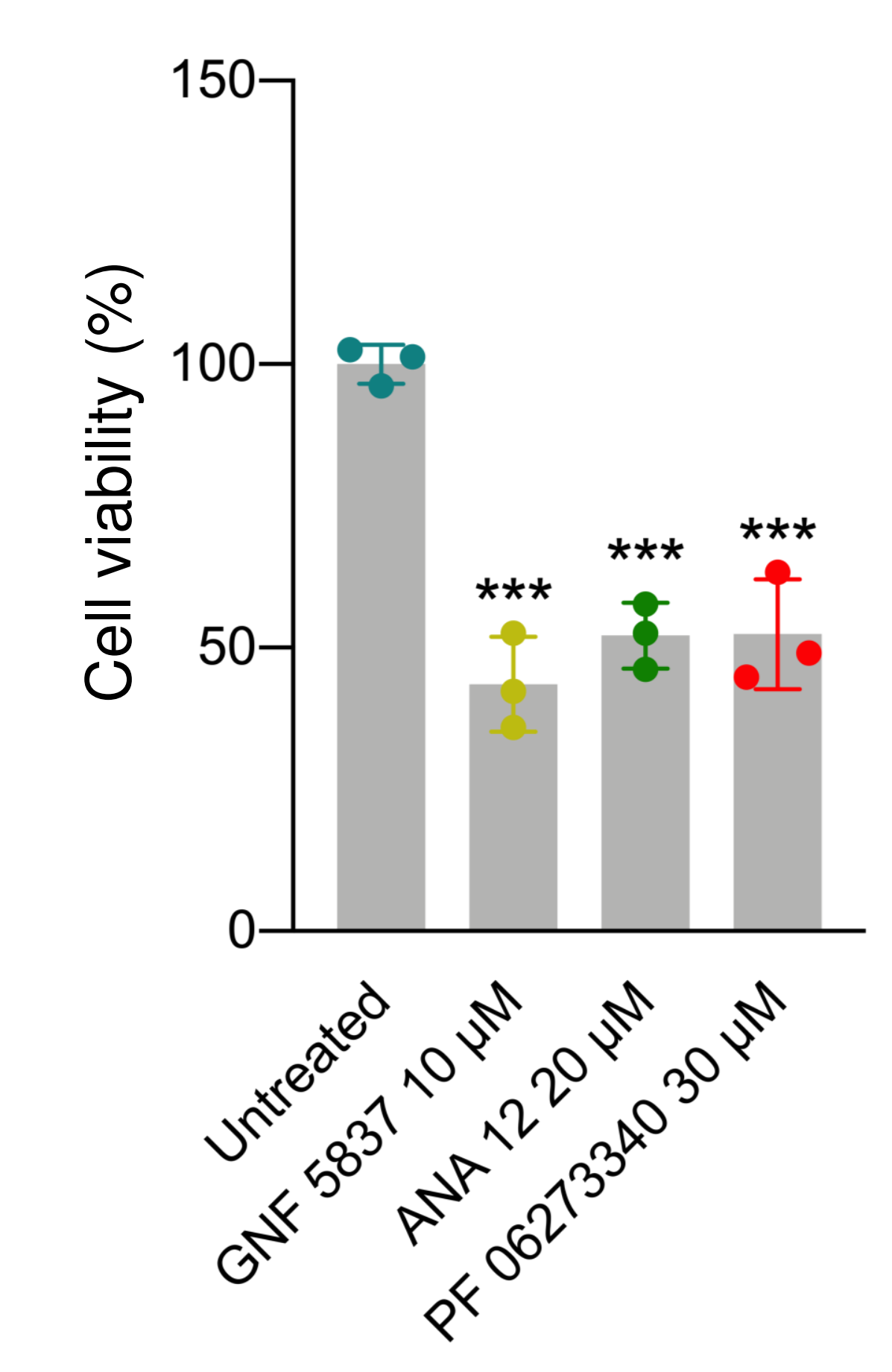

d

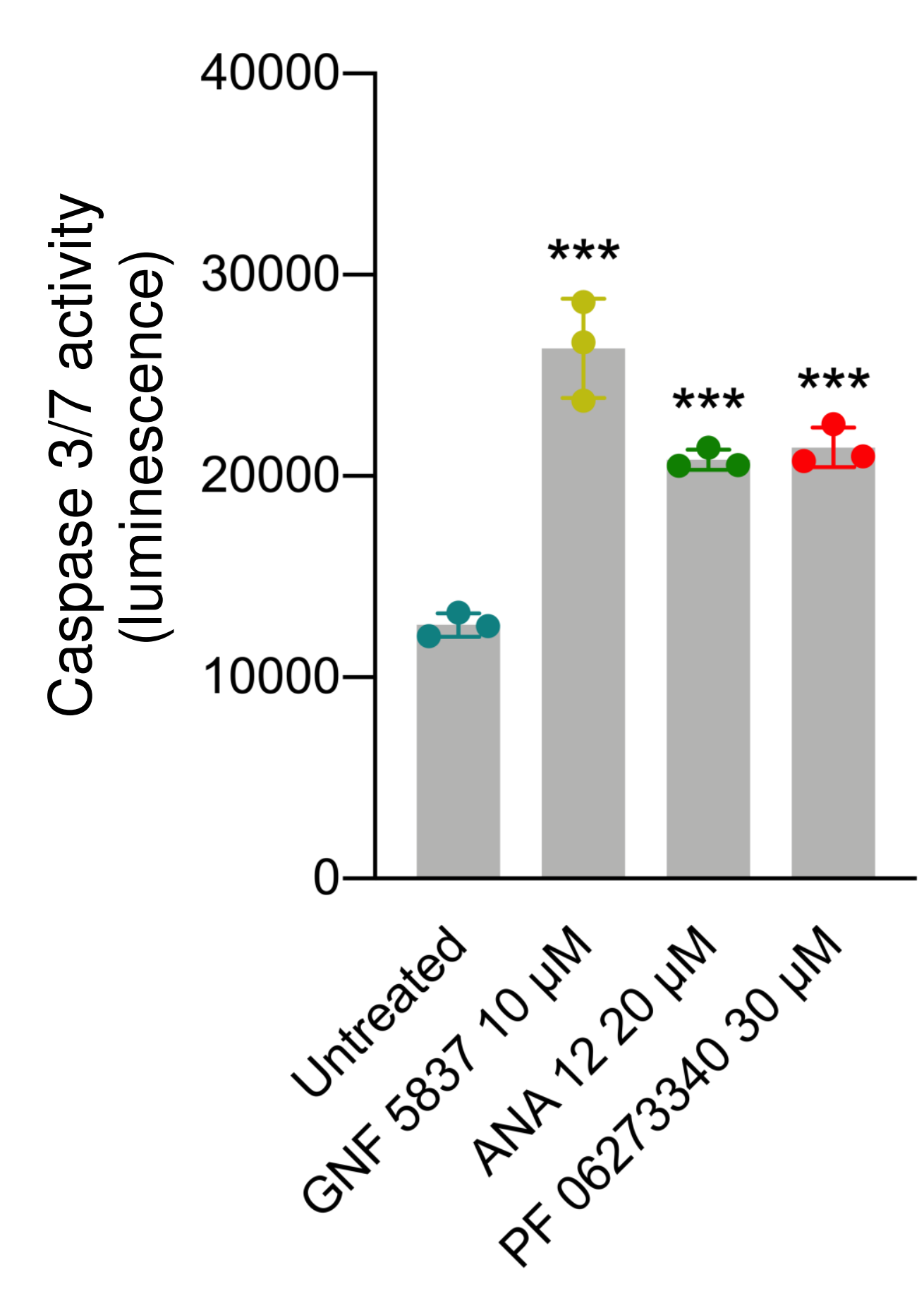

g

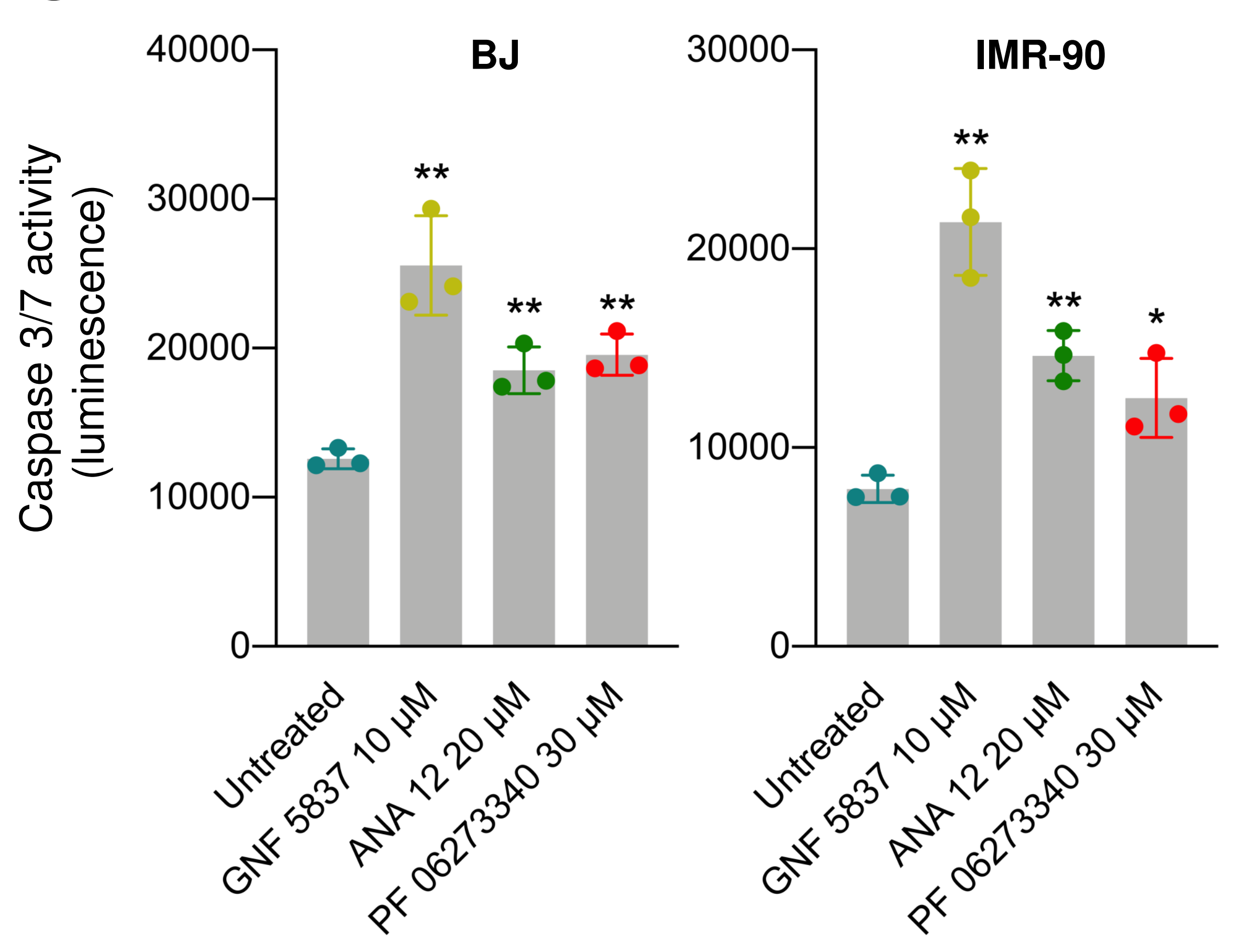

e

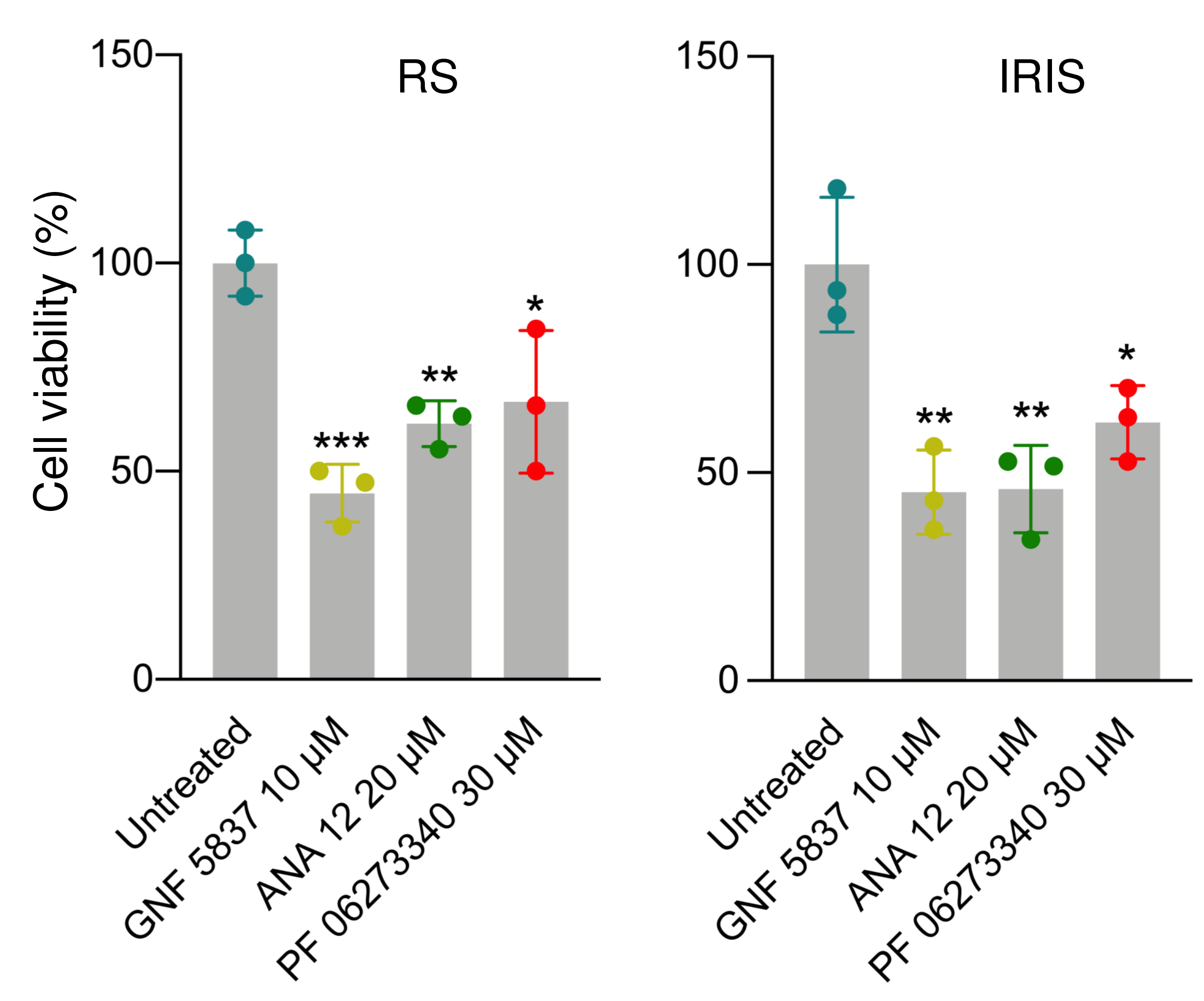

h

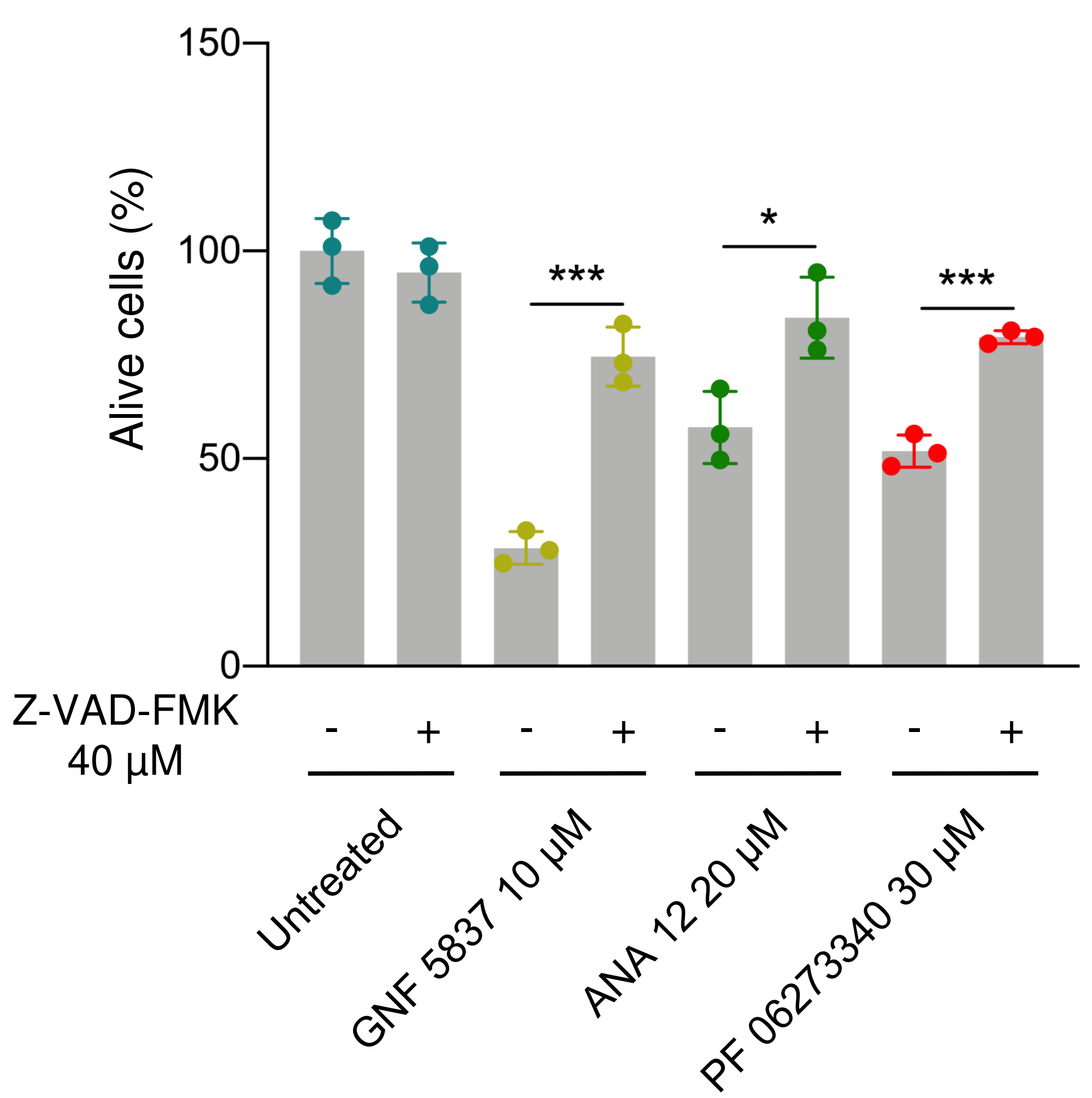

f

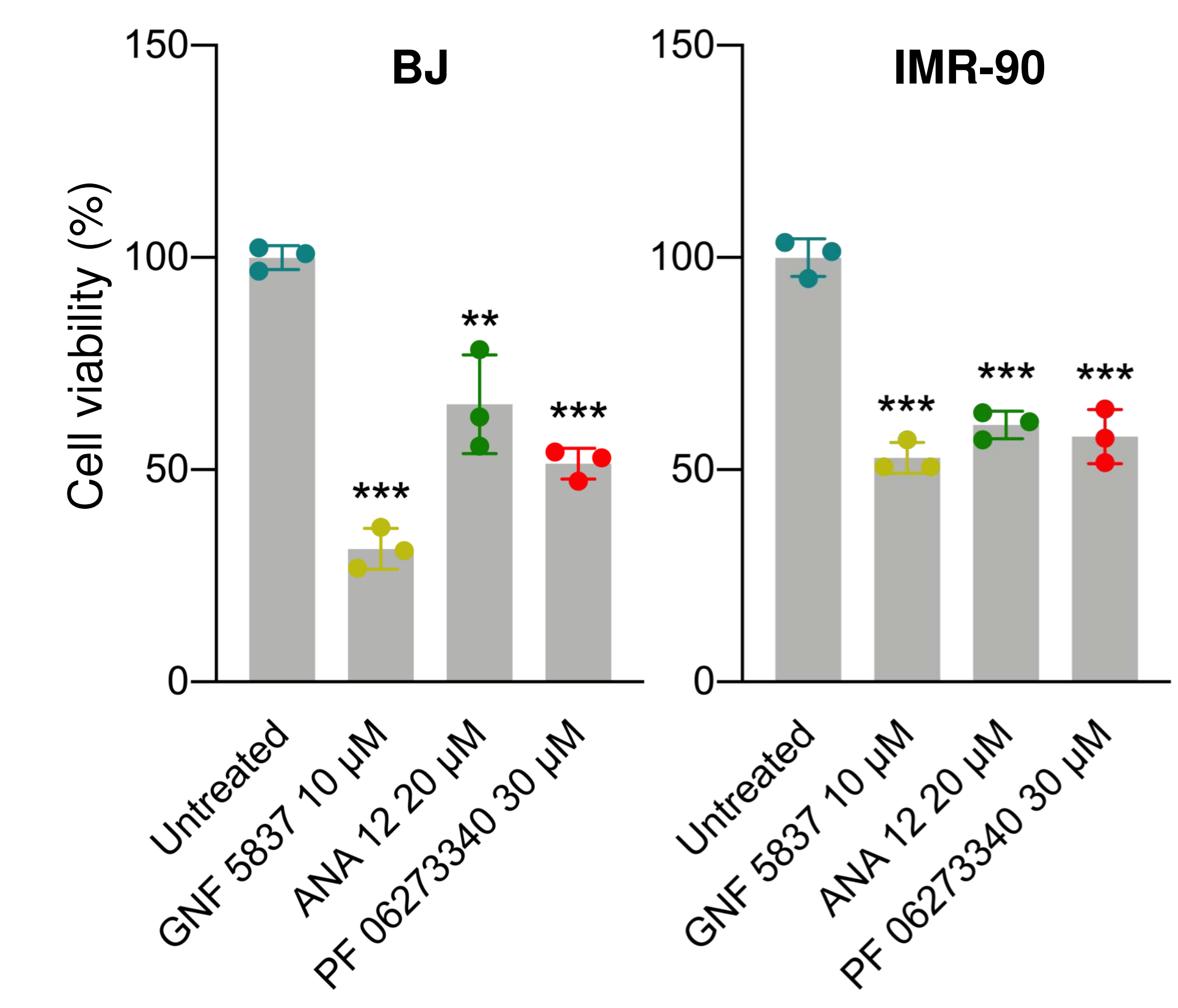

i

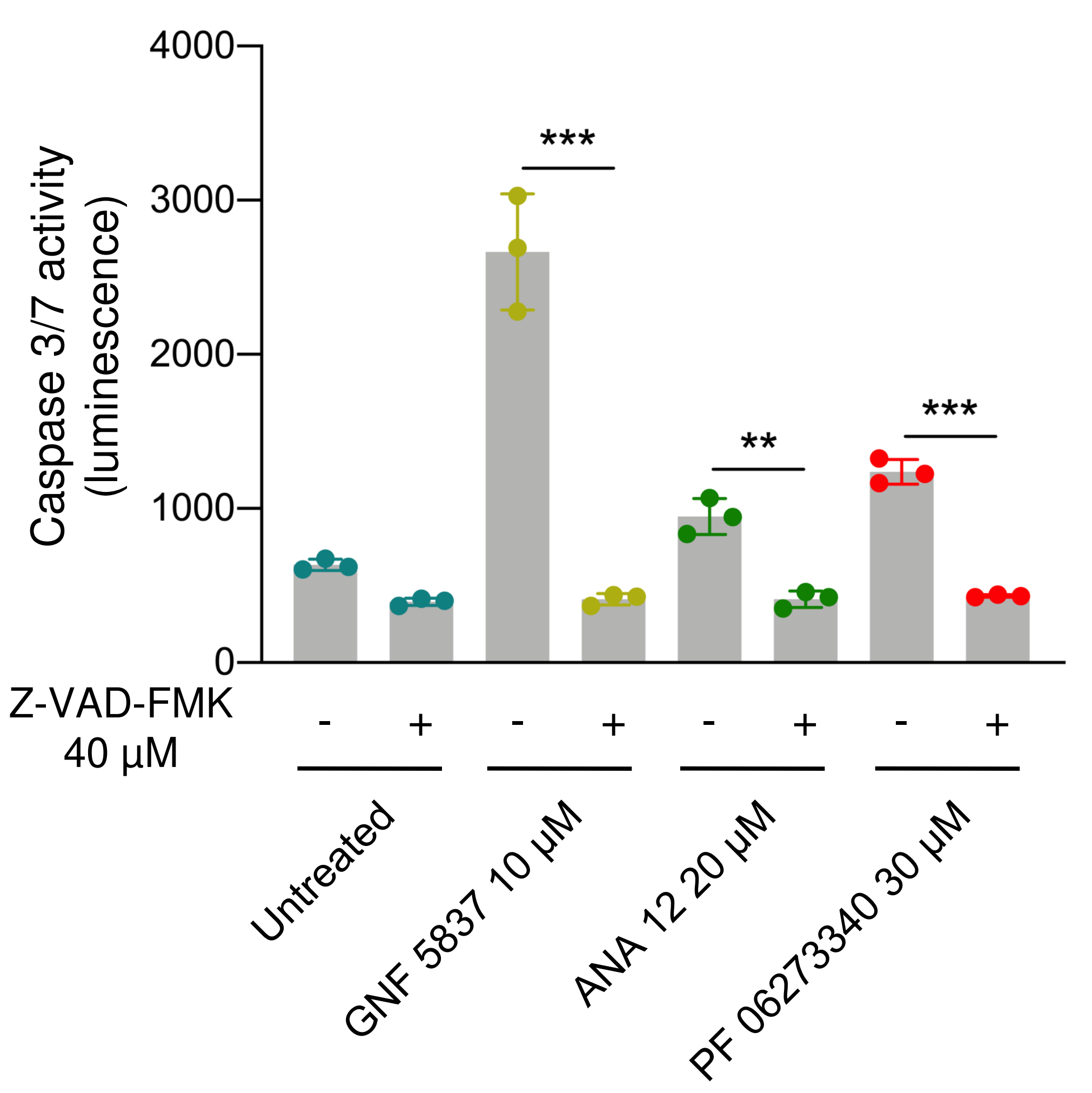


a

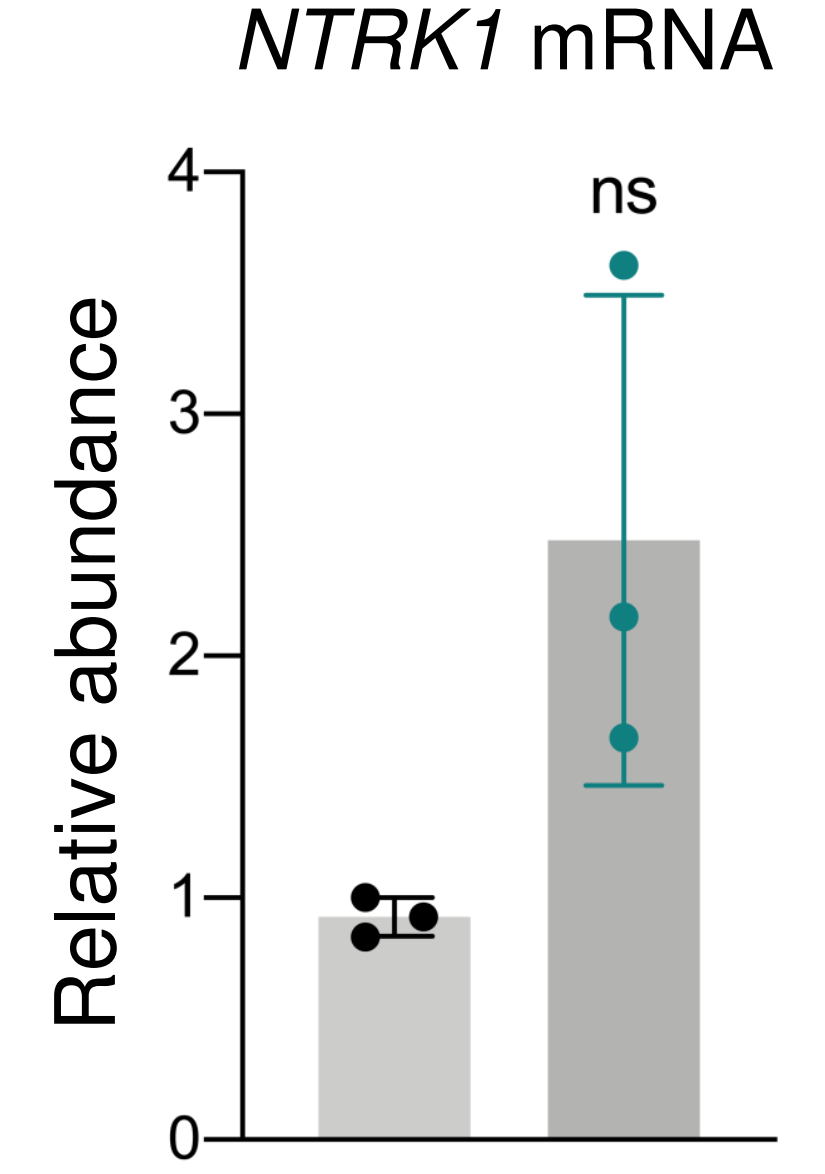

P S
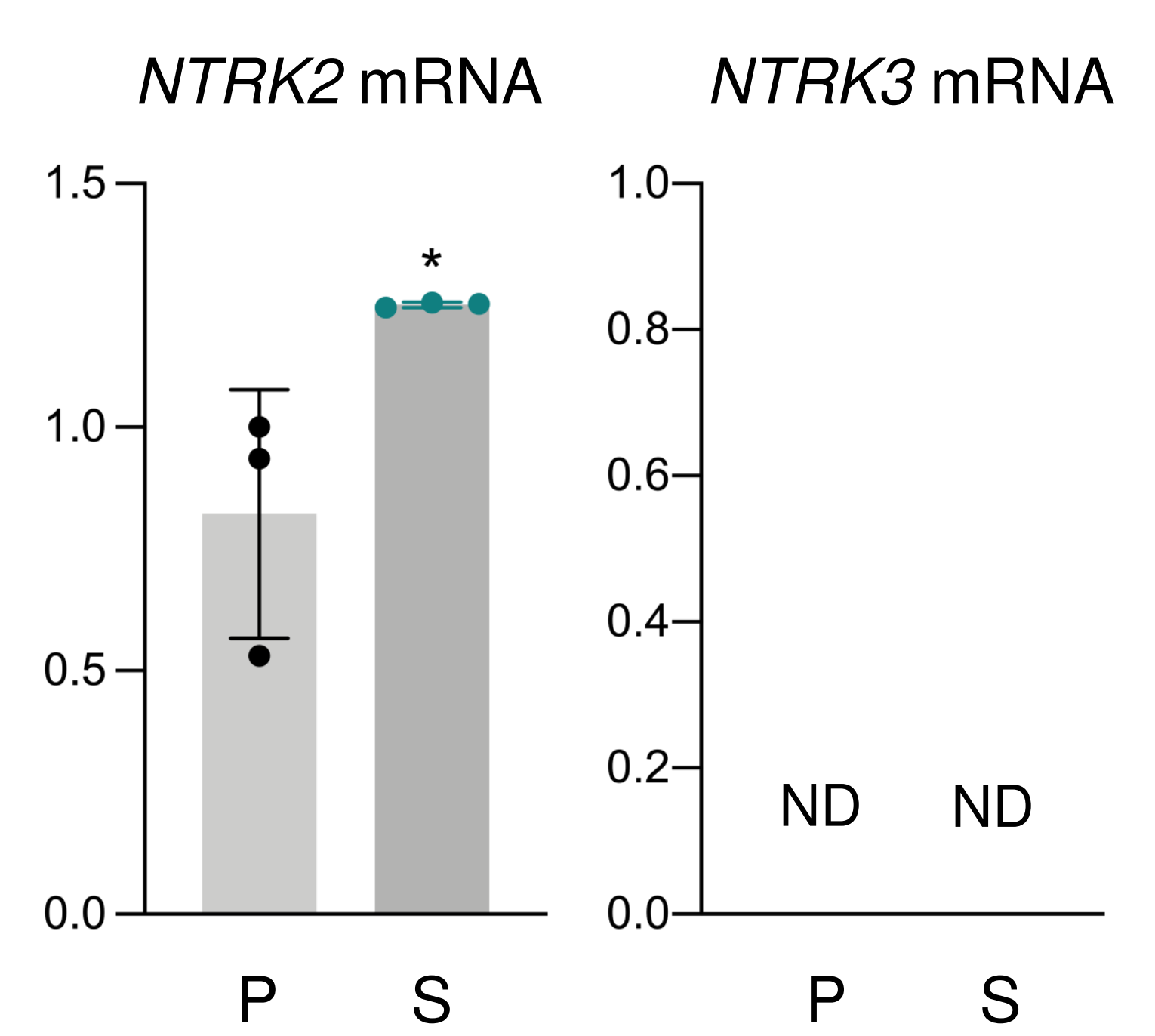

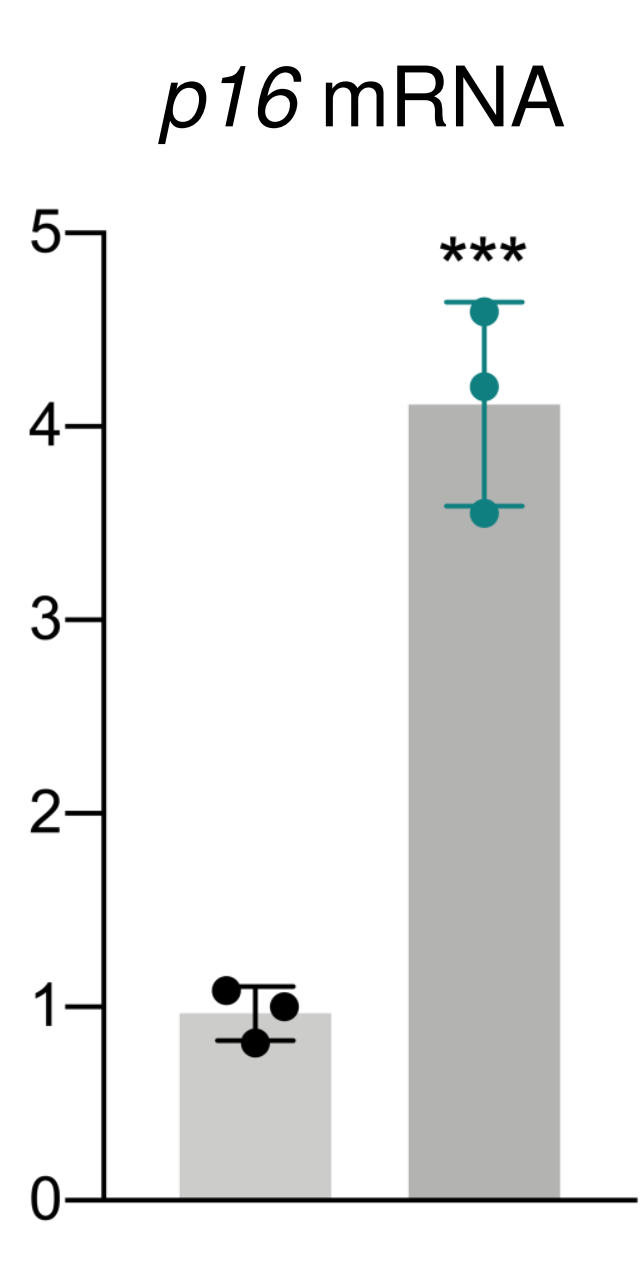

P $S$

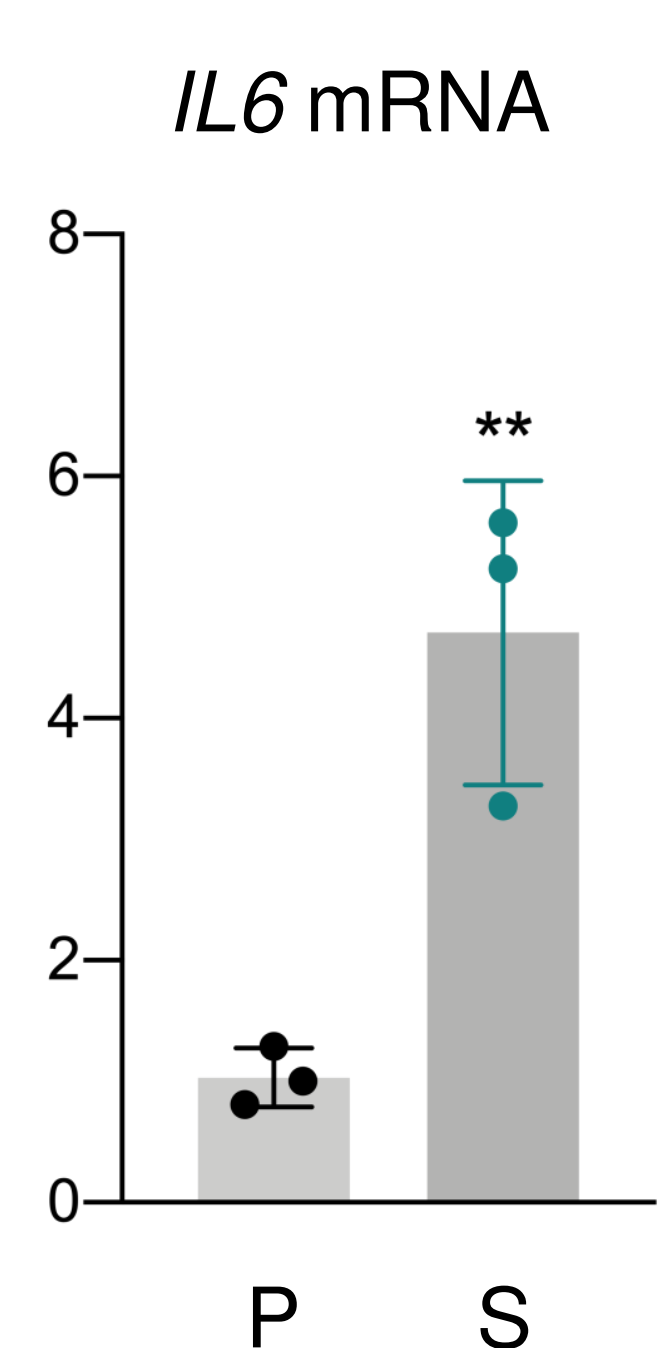

b

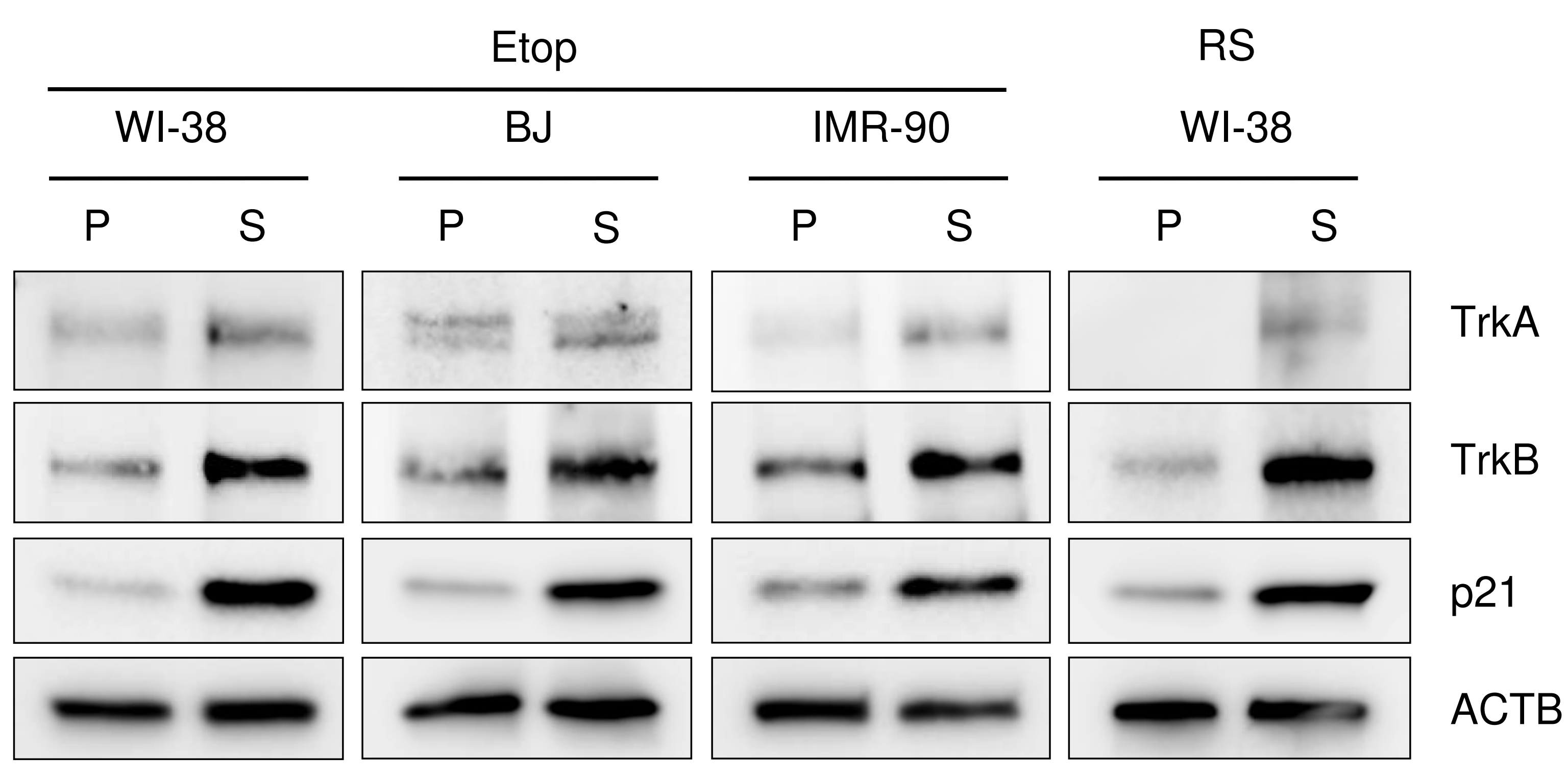

e
C

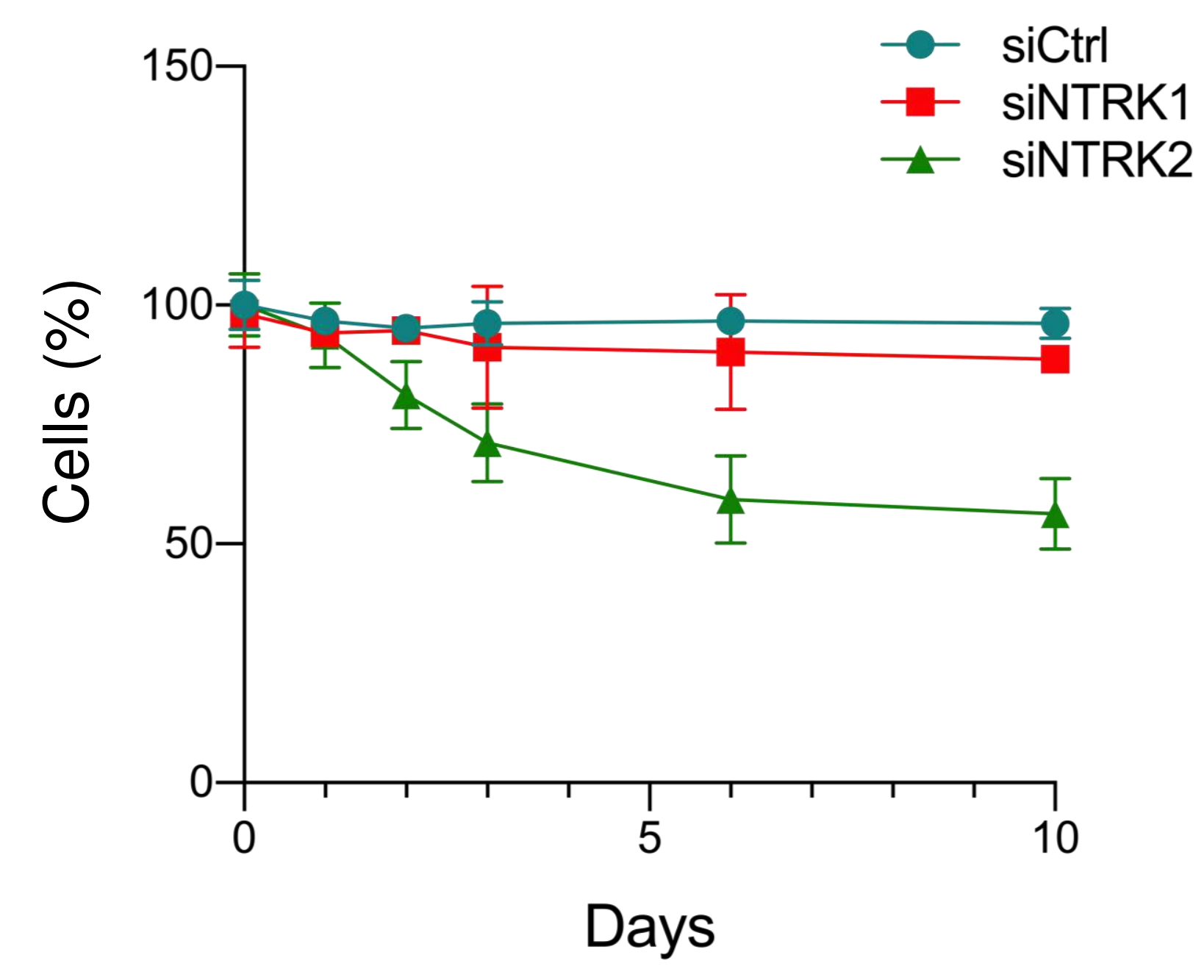

d

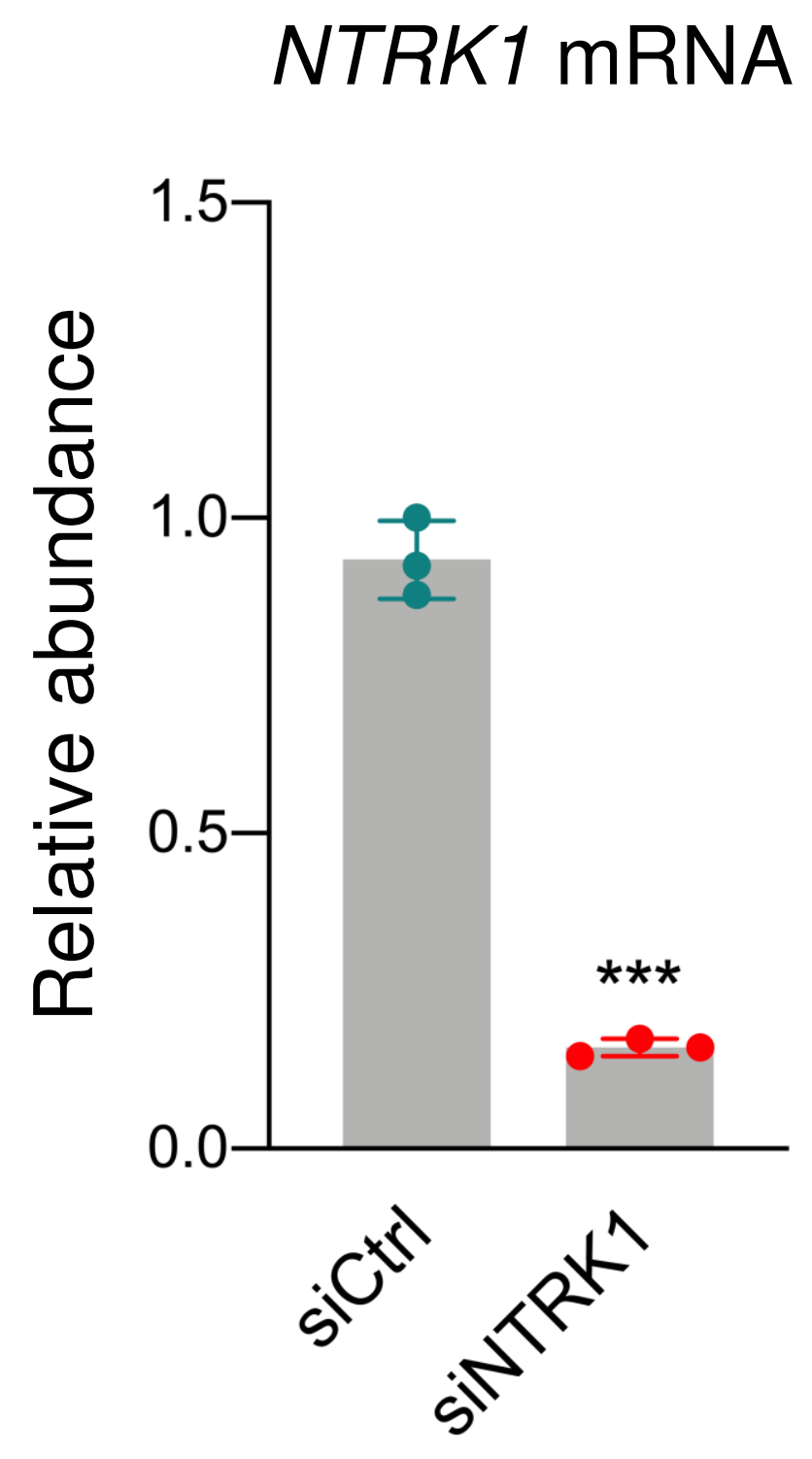

NTRK2 mRNA

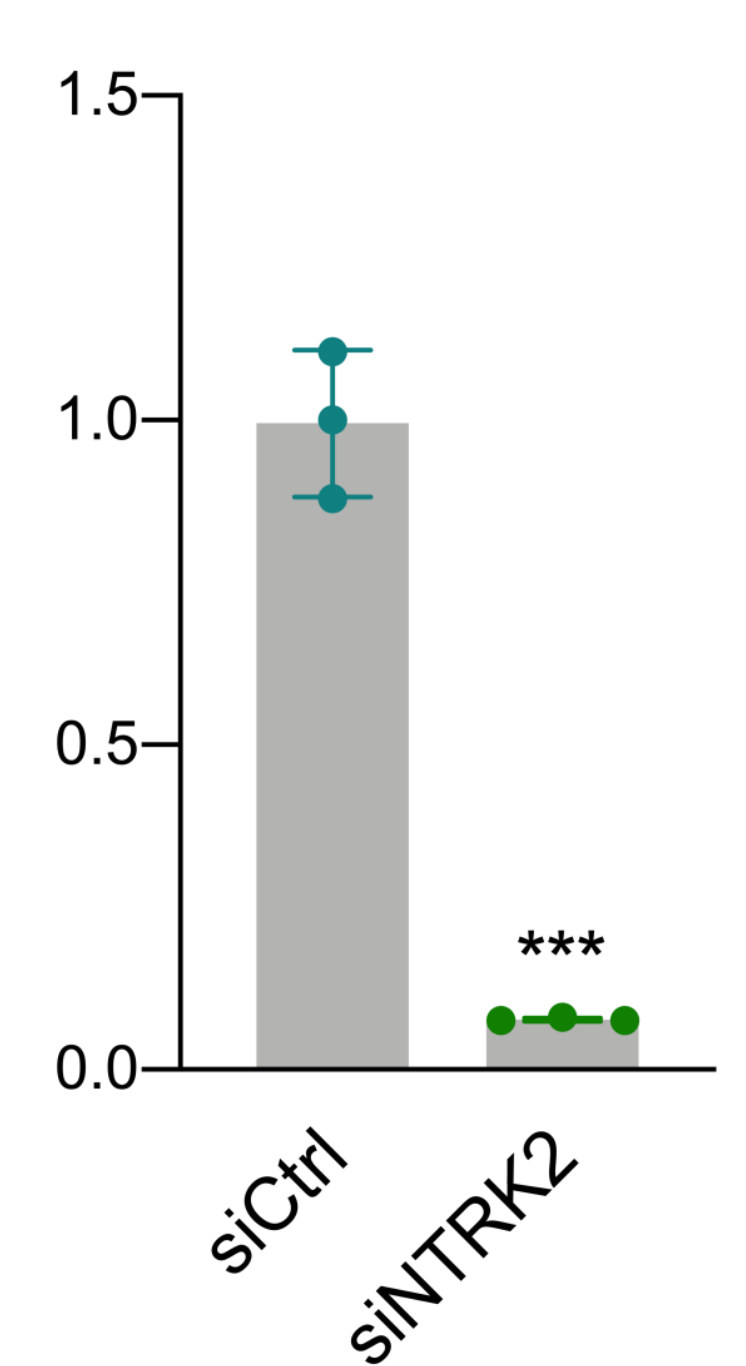

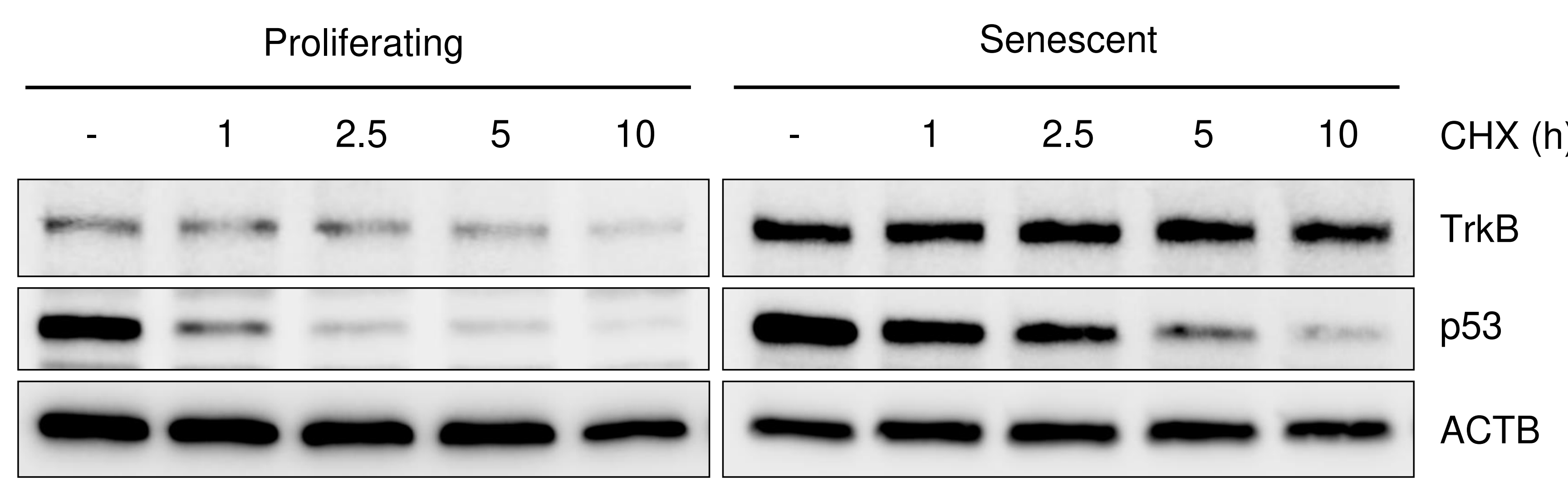

h

g

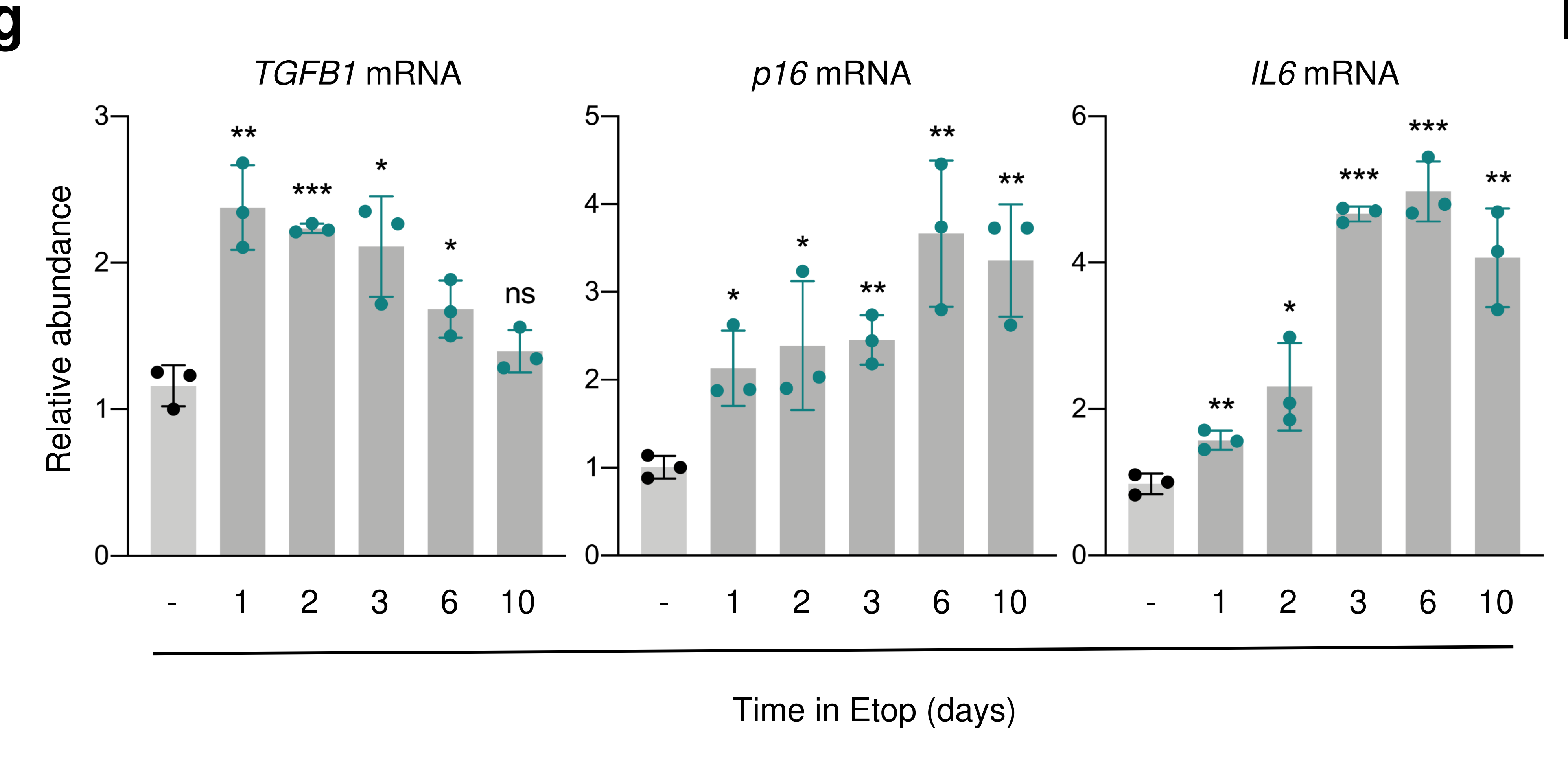

Surface Pulldown

P S

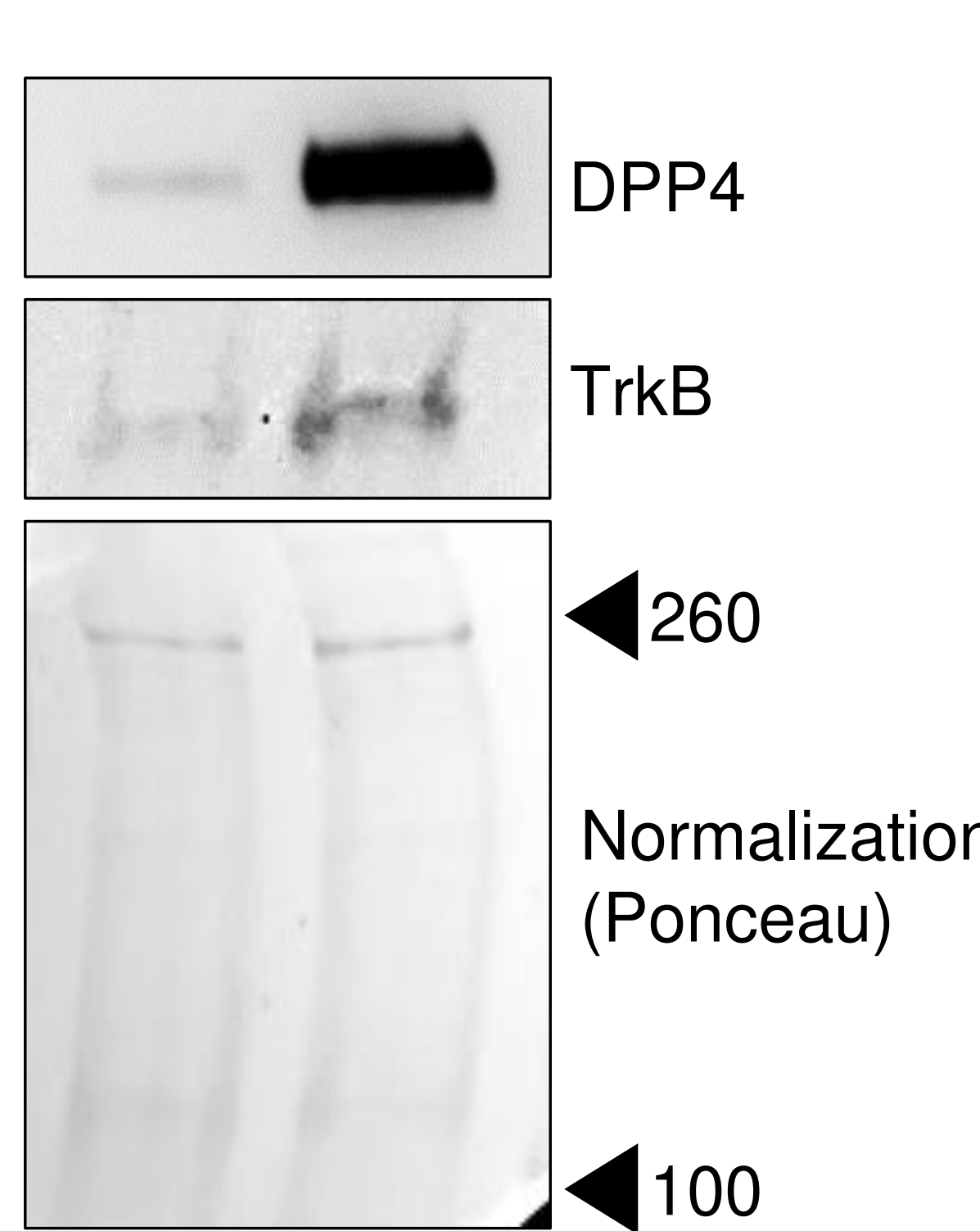

i

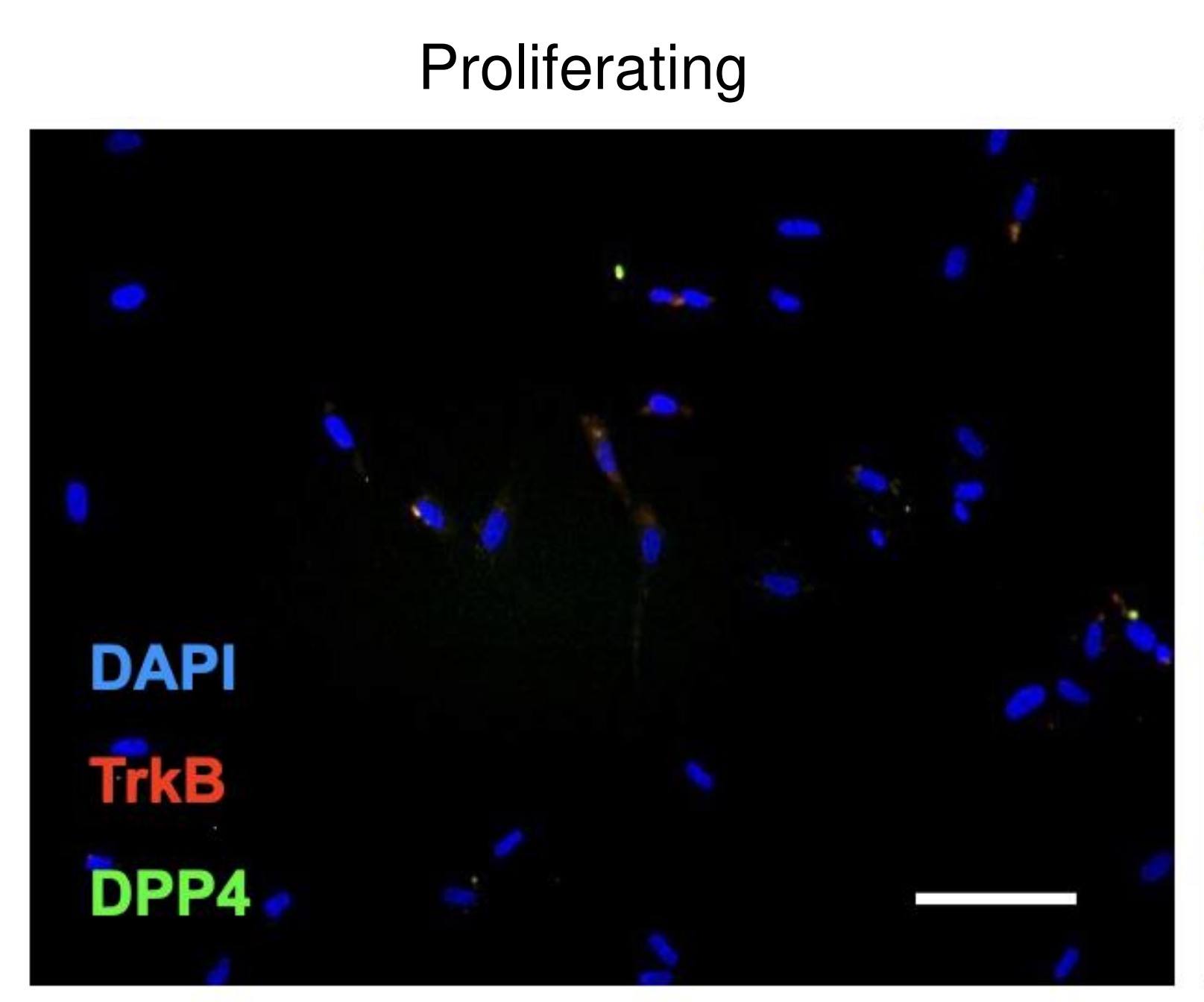

Senescent

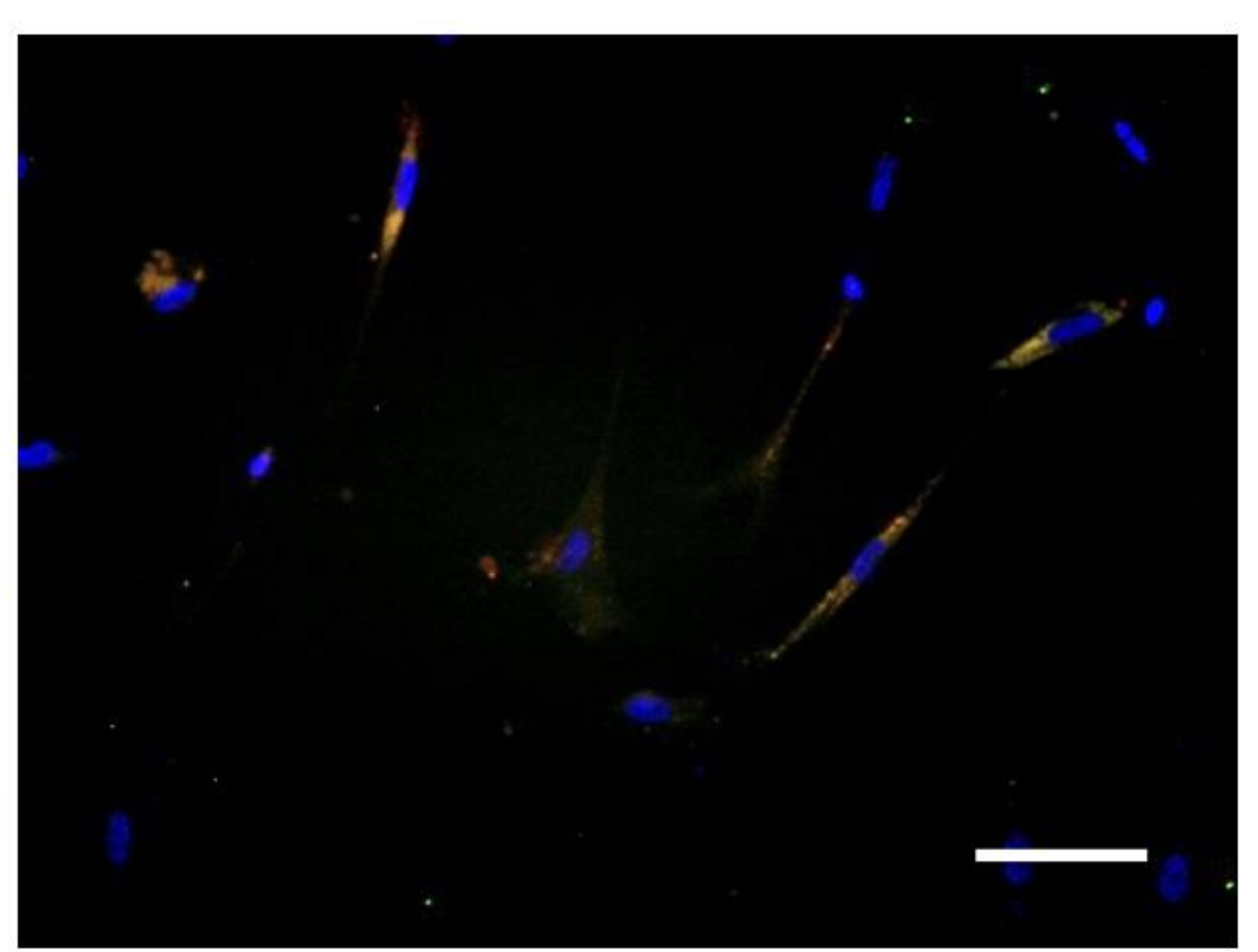

$20 x$
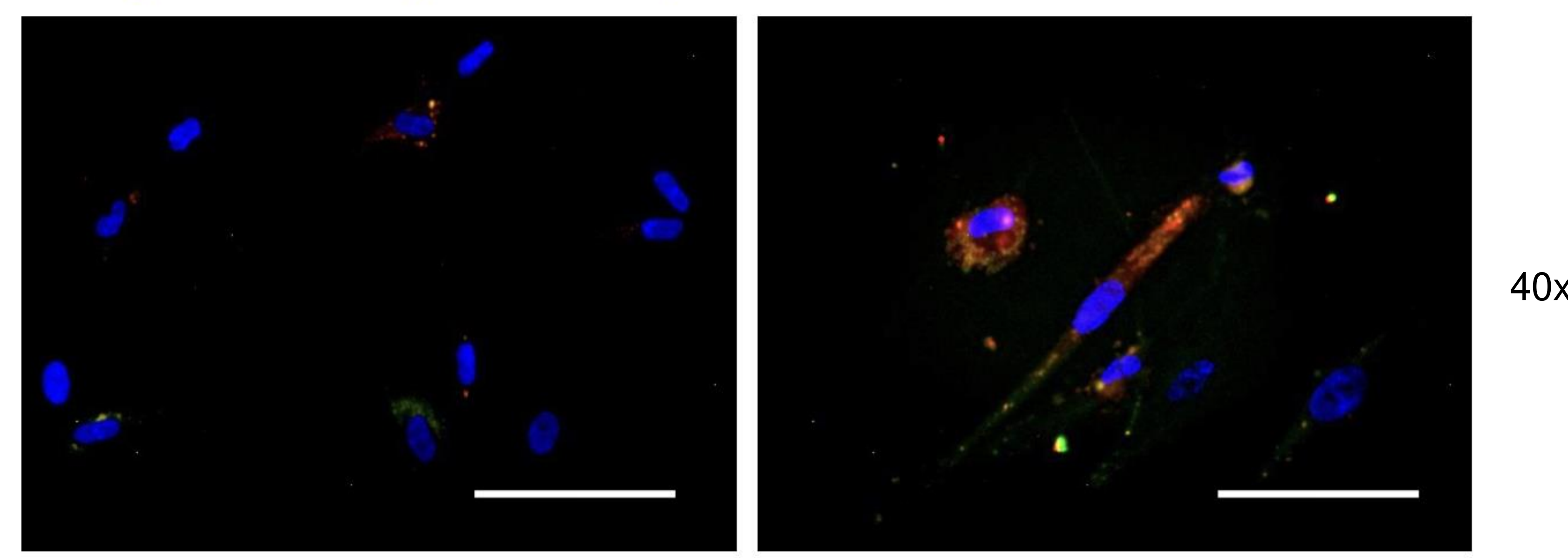
a

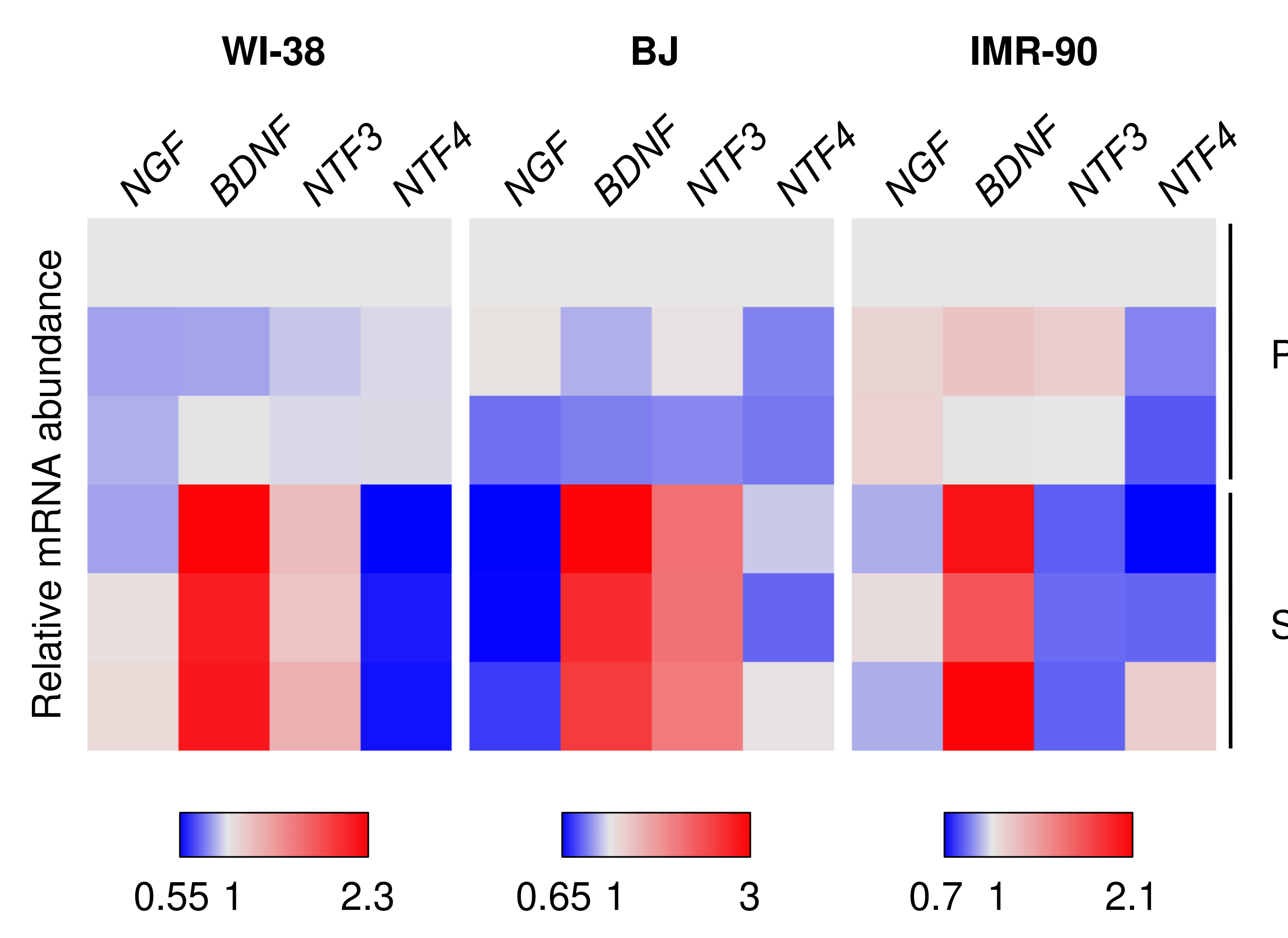

d
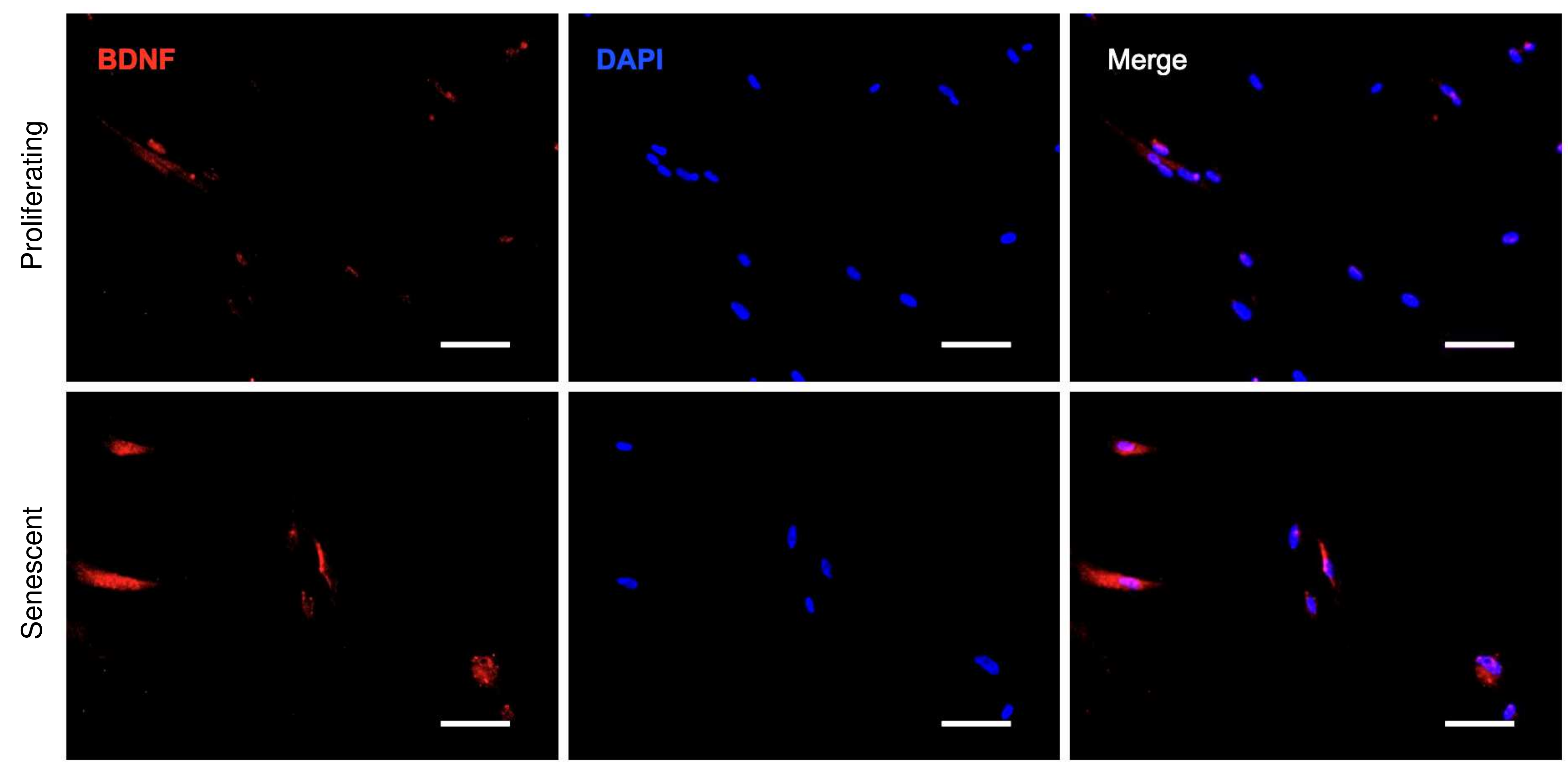

C

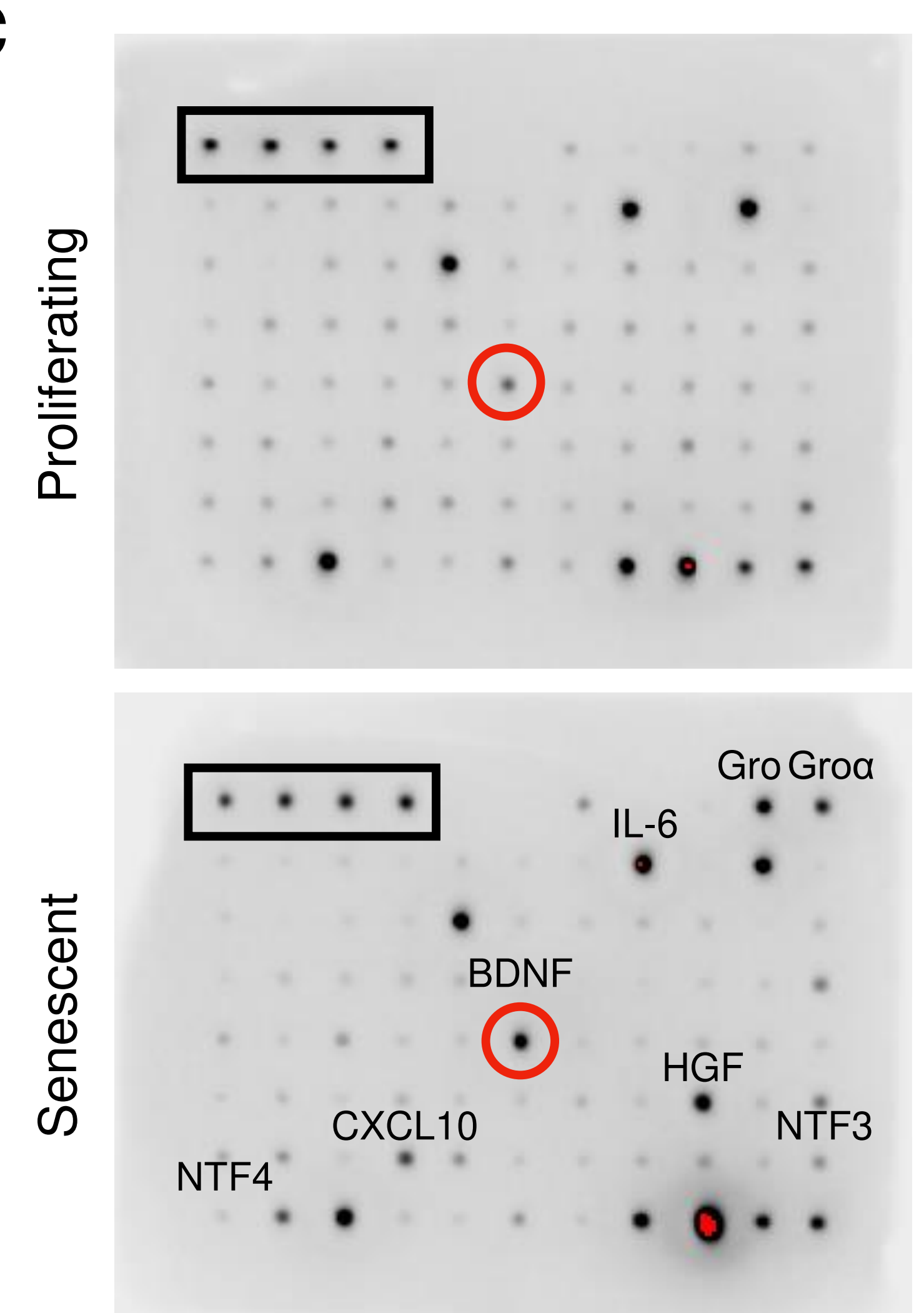

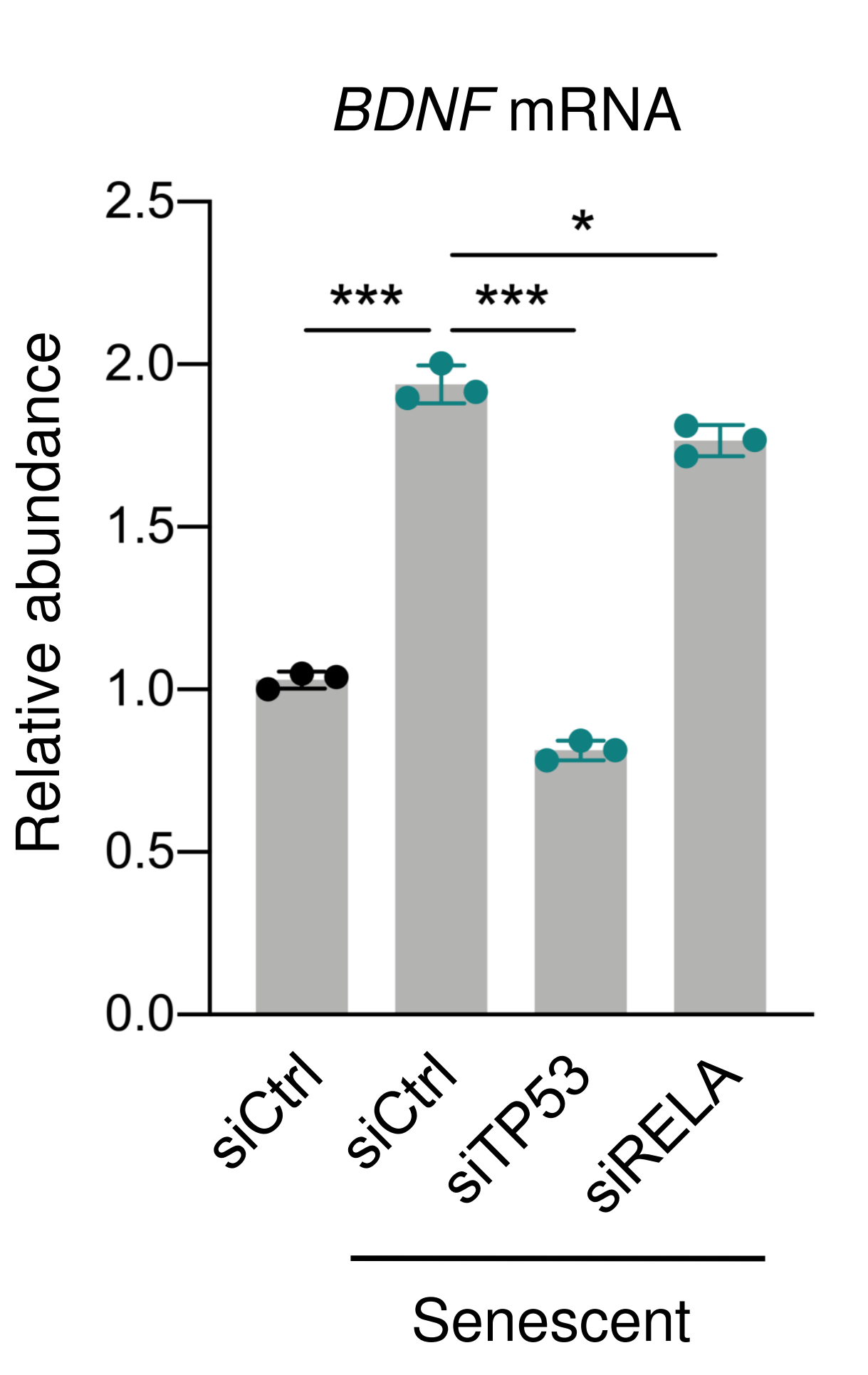
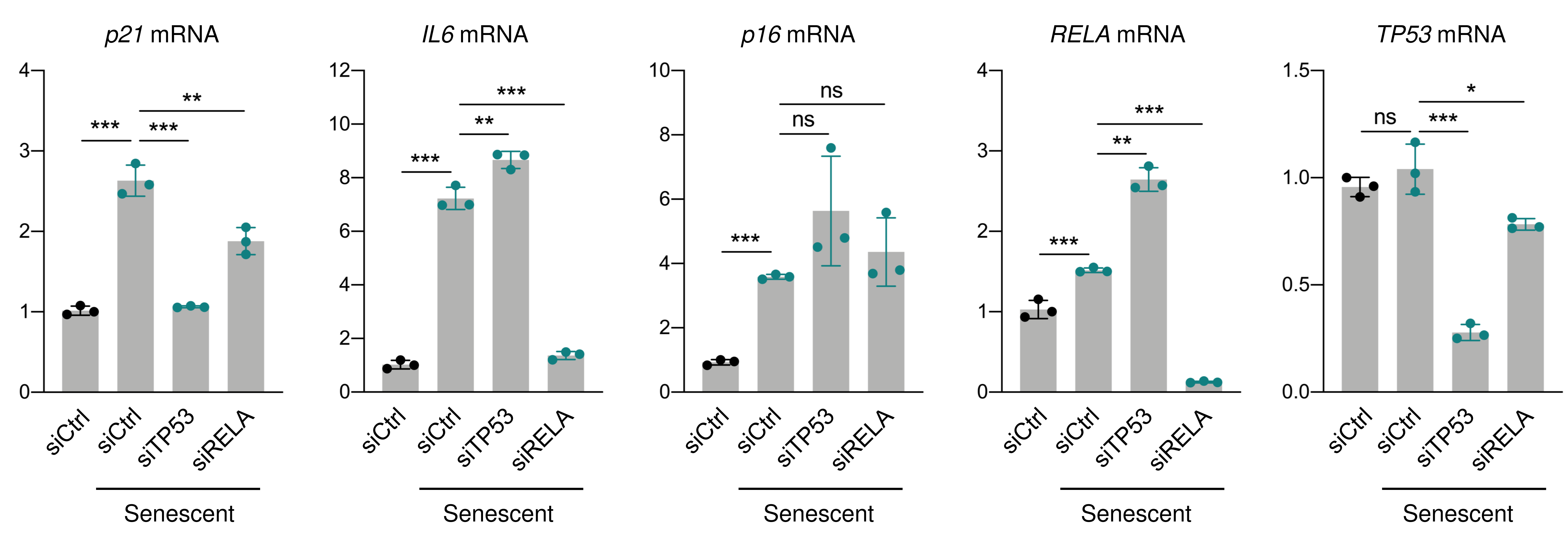

g
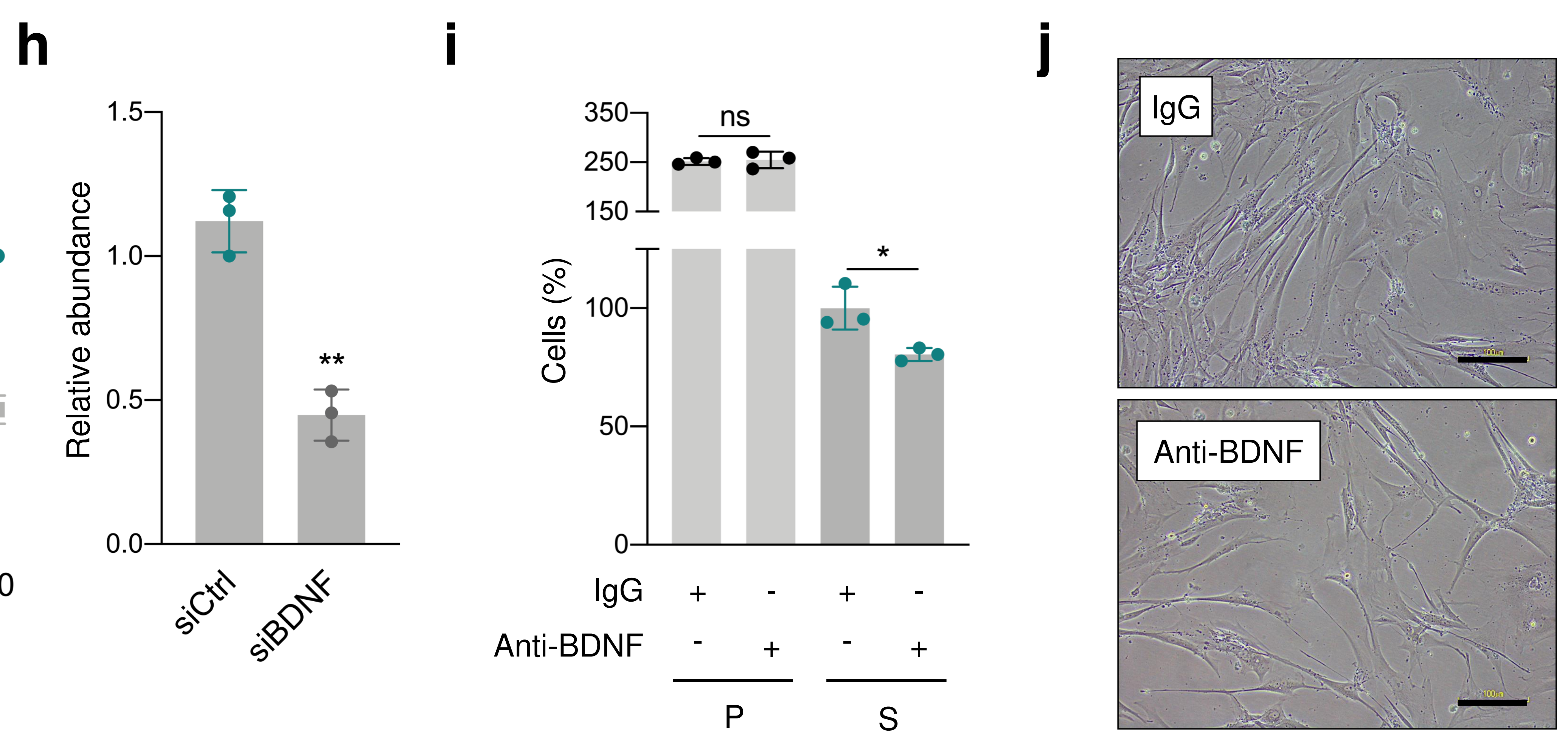

k
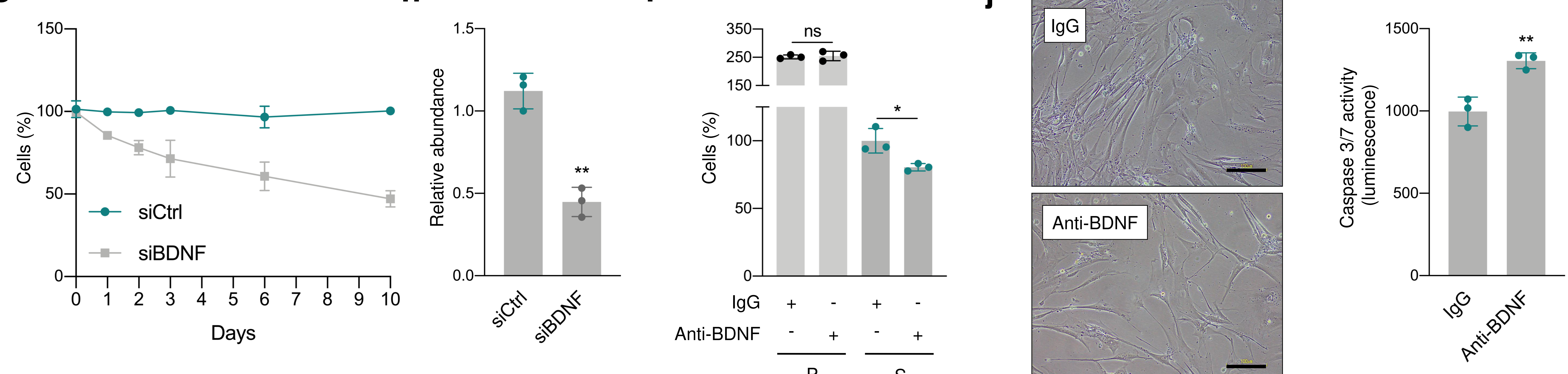
a

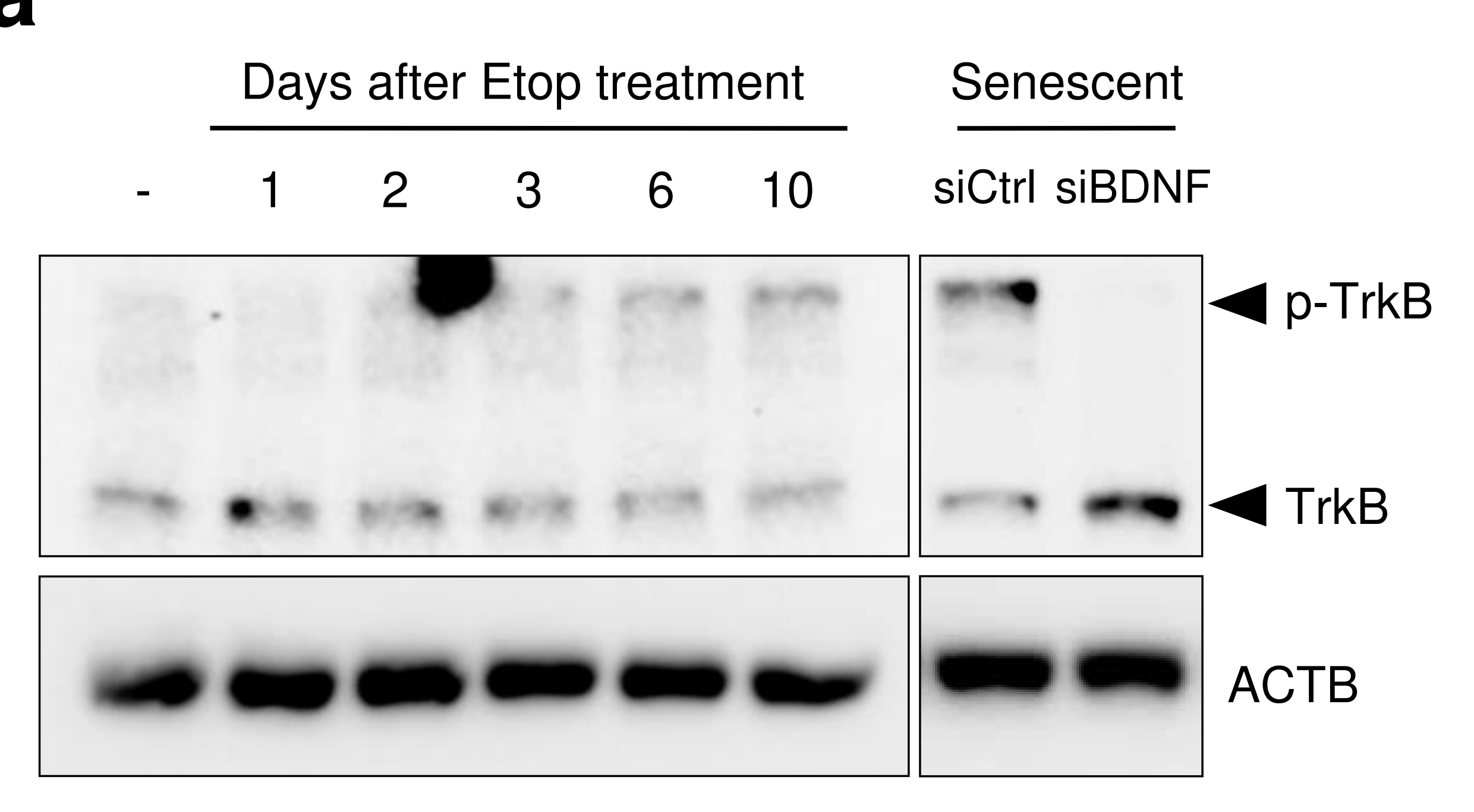

b

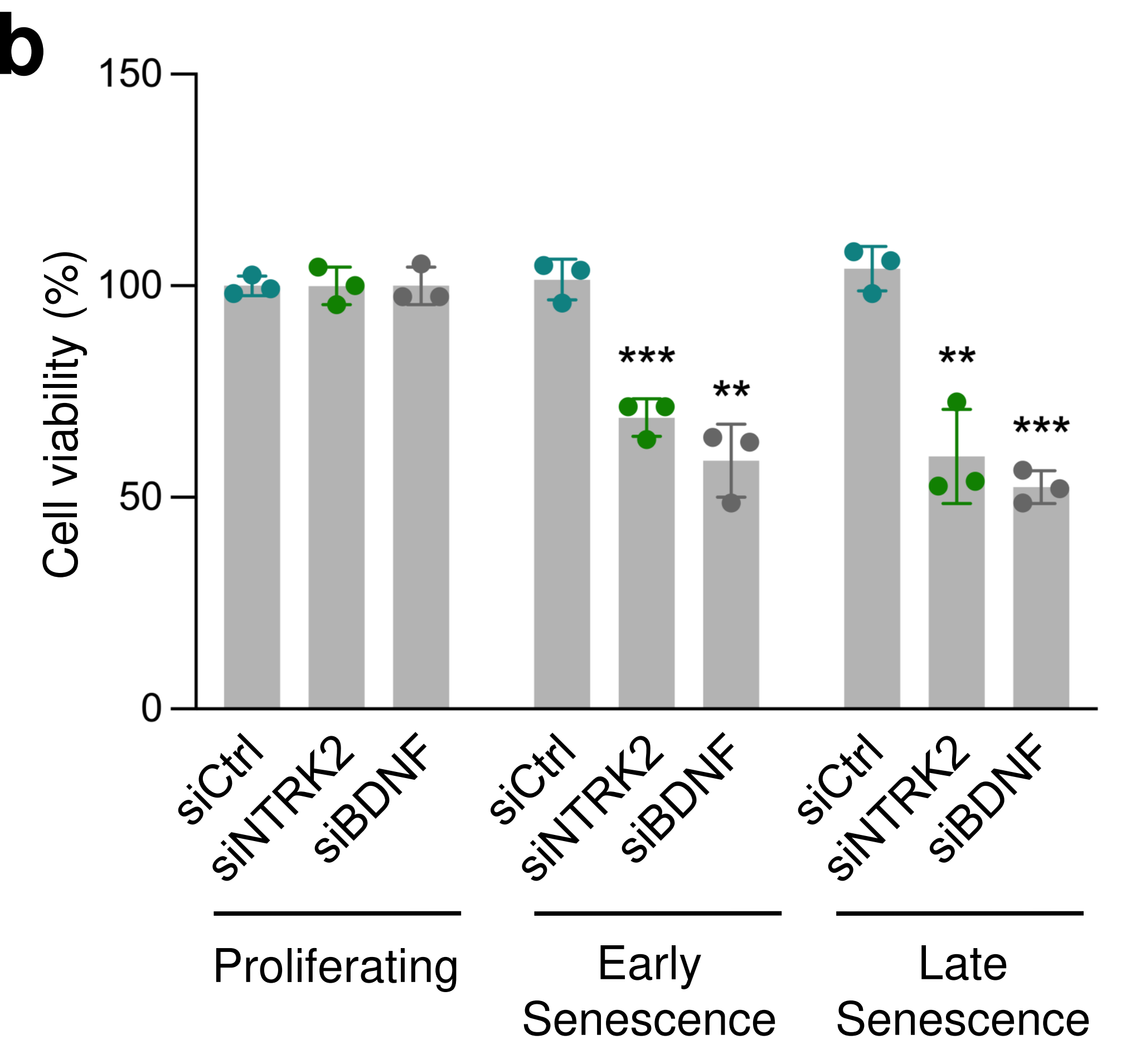

C

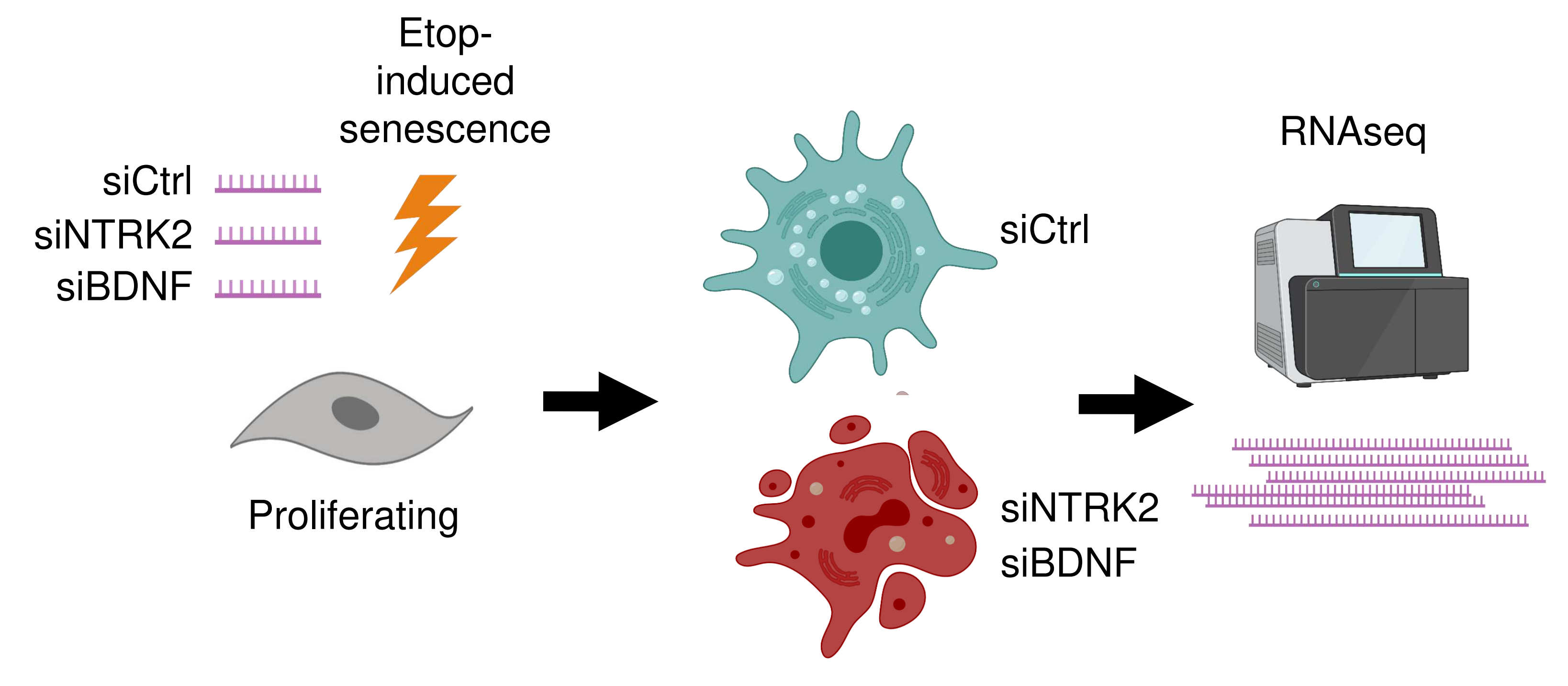

d
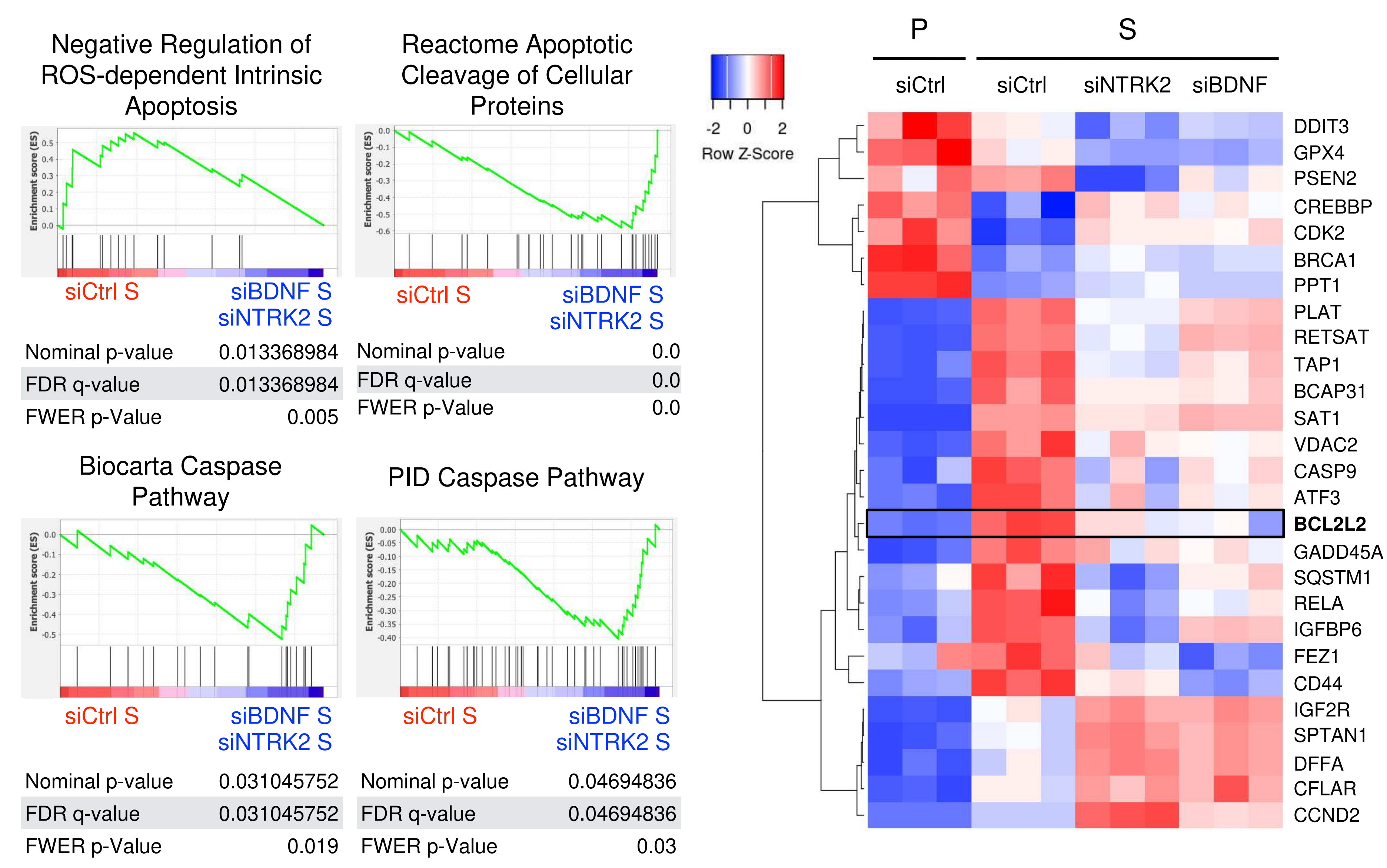

e

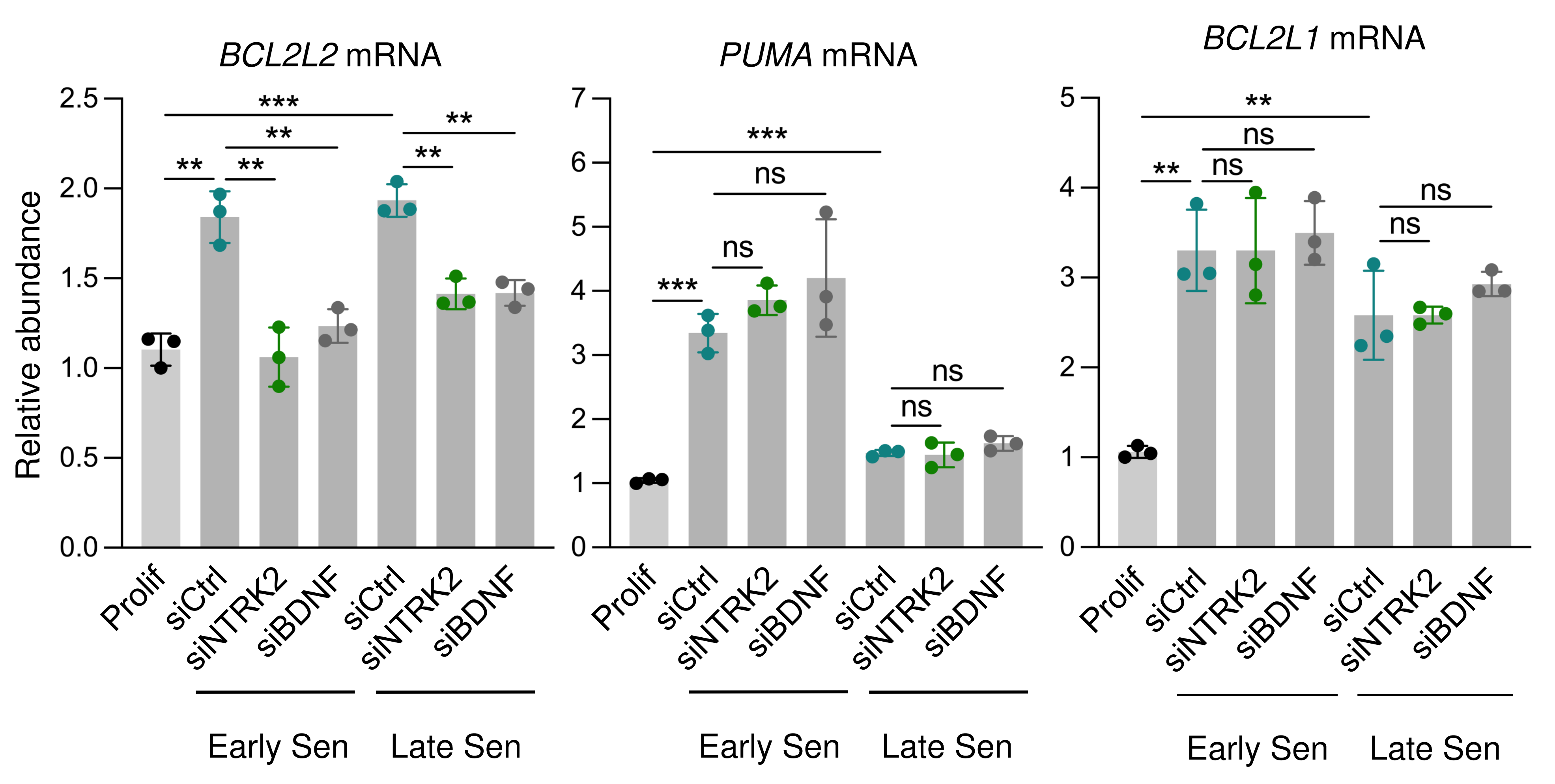

f

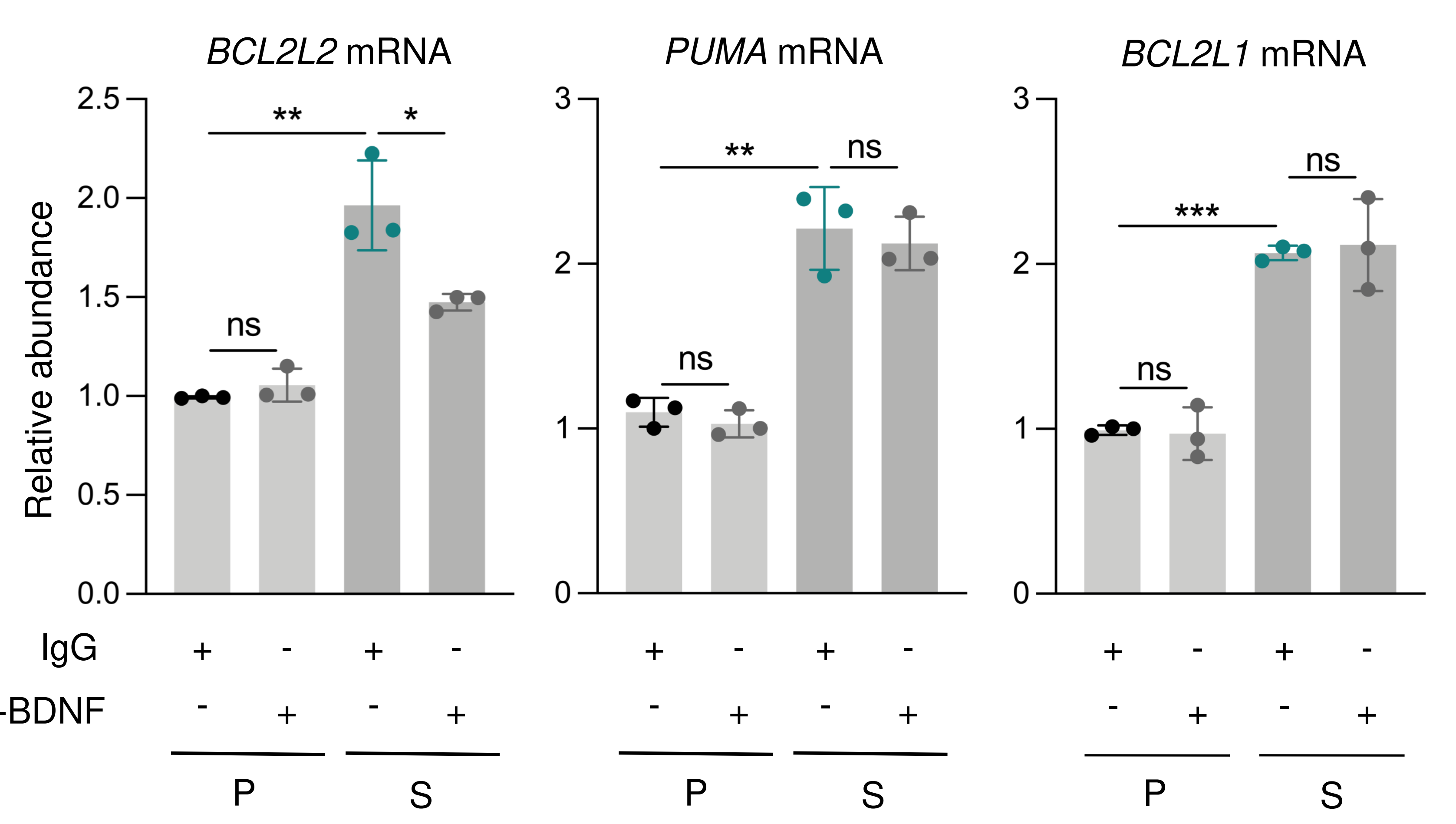

g

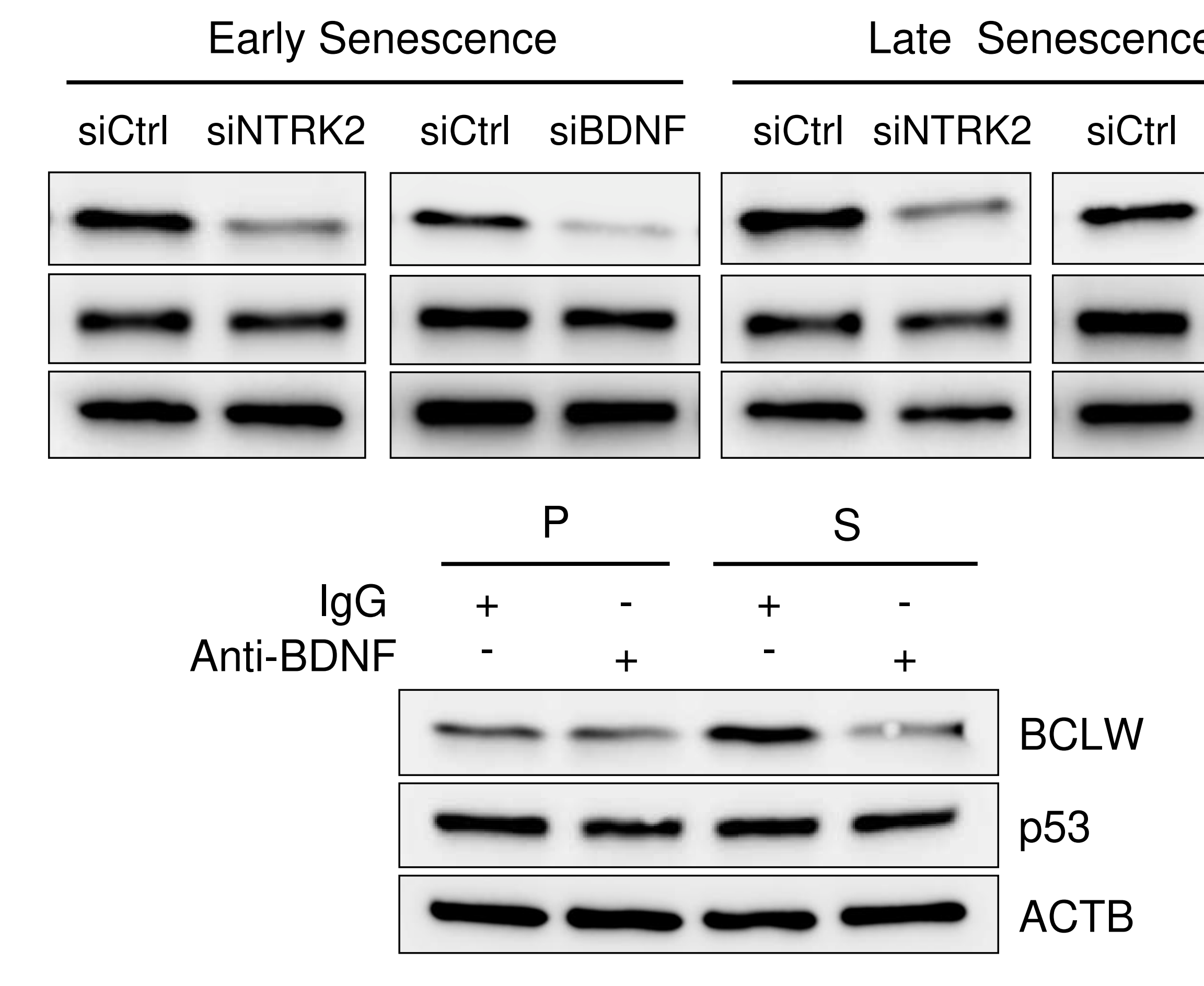

h

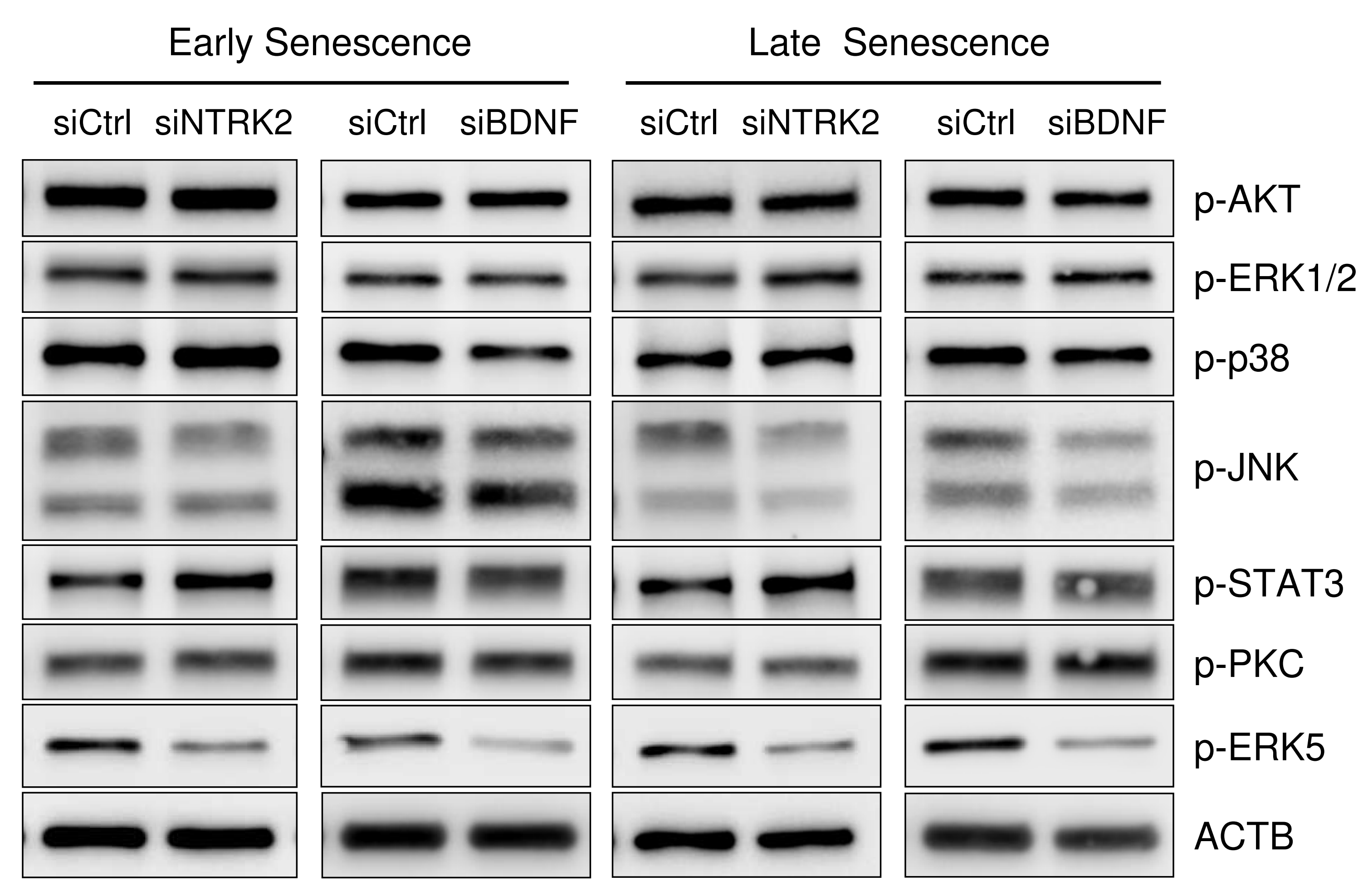

i

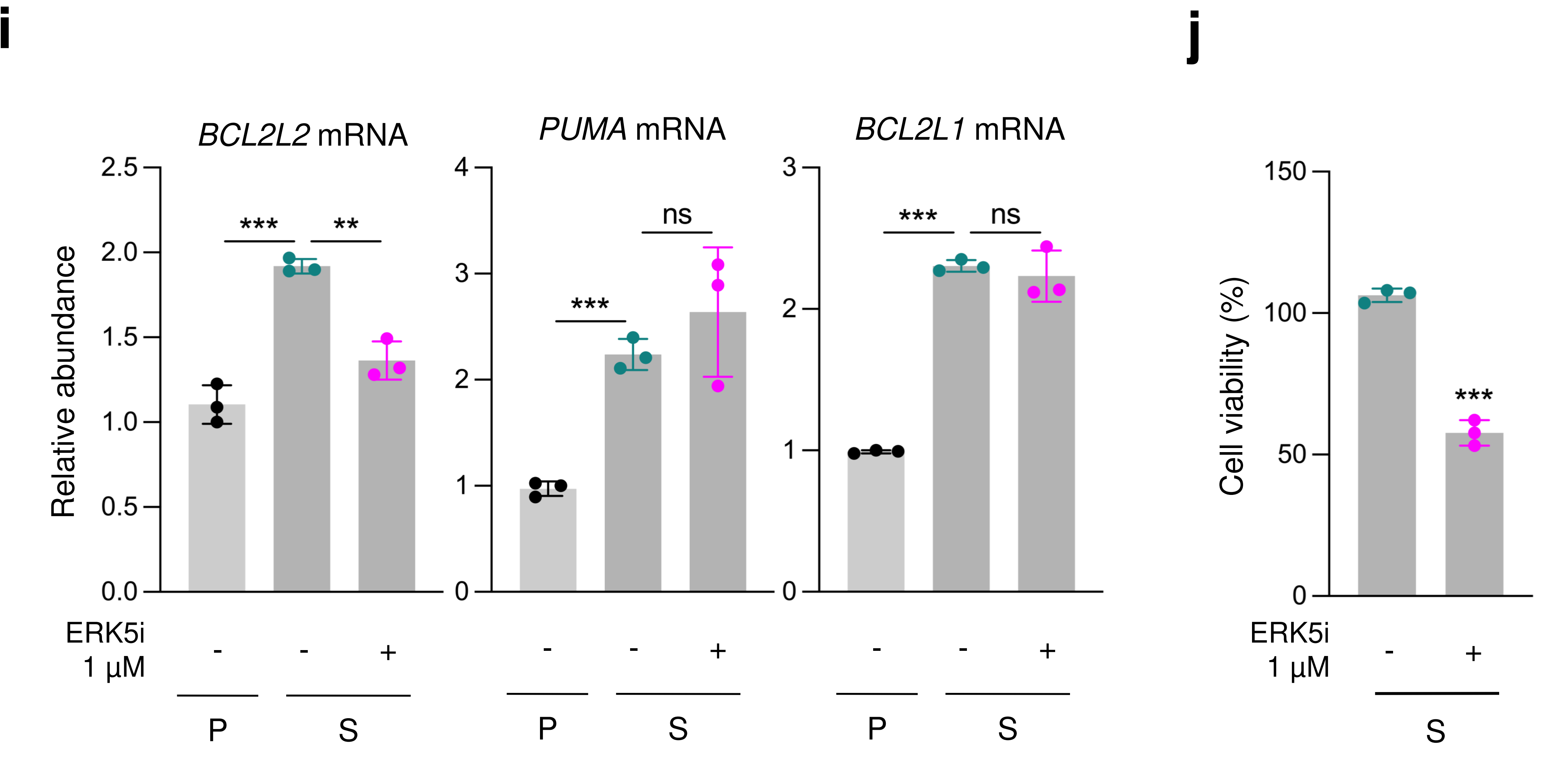


Anerillas et al. Figure 6

a

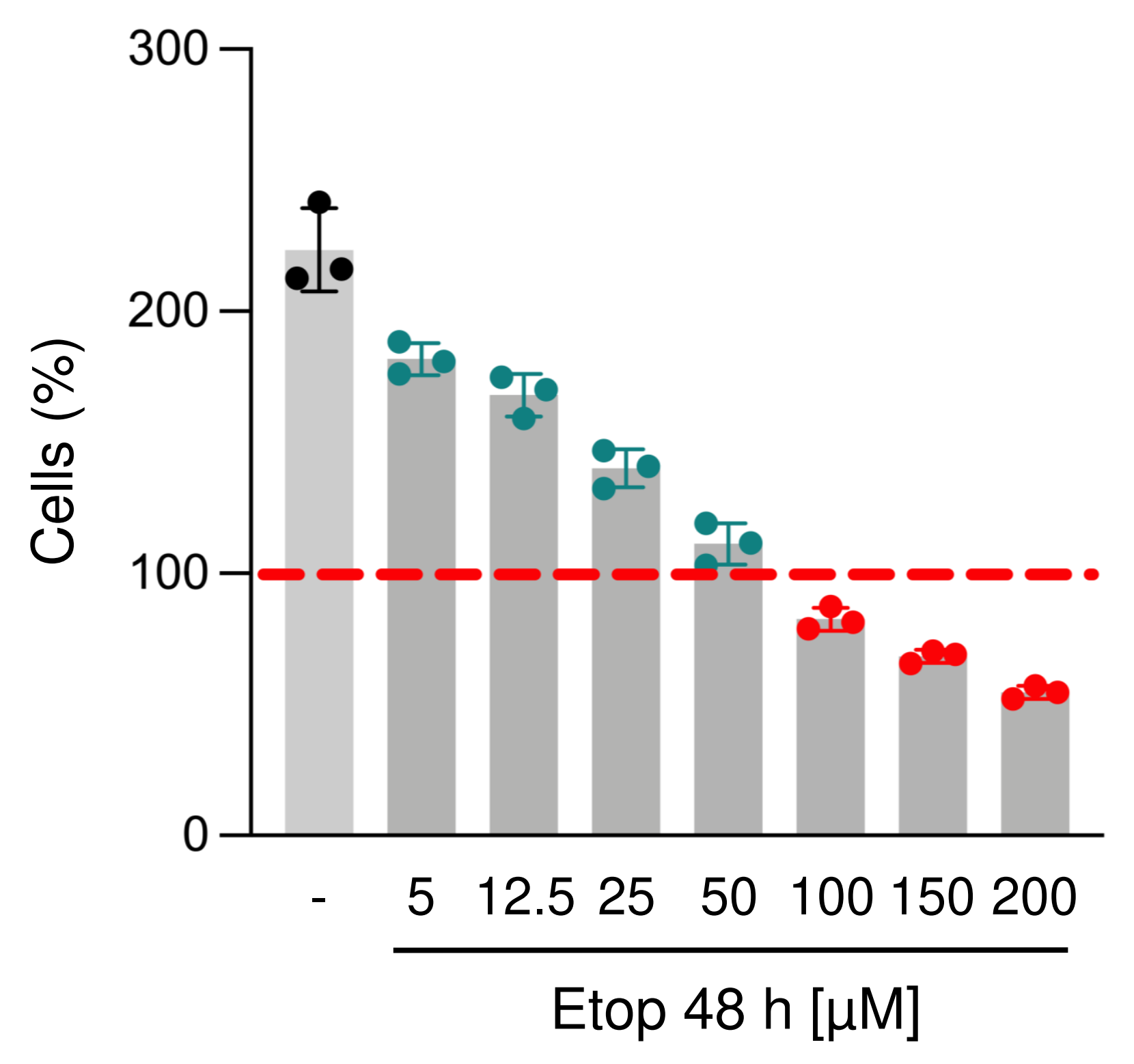

d

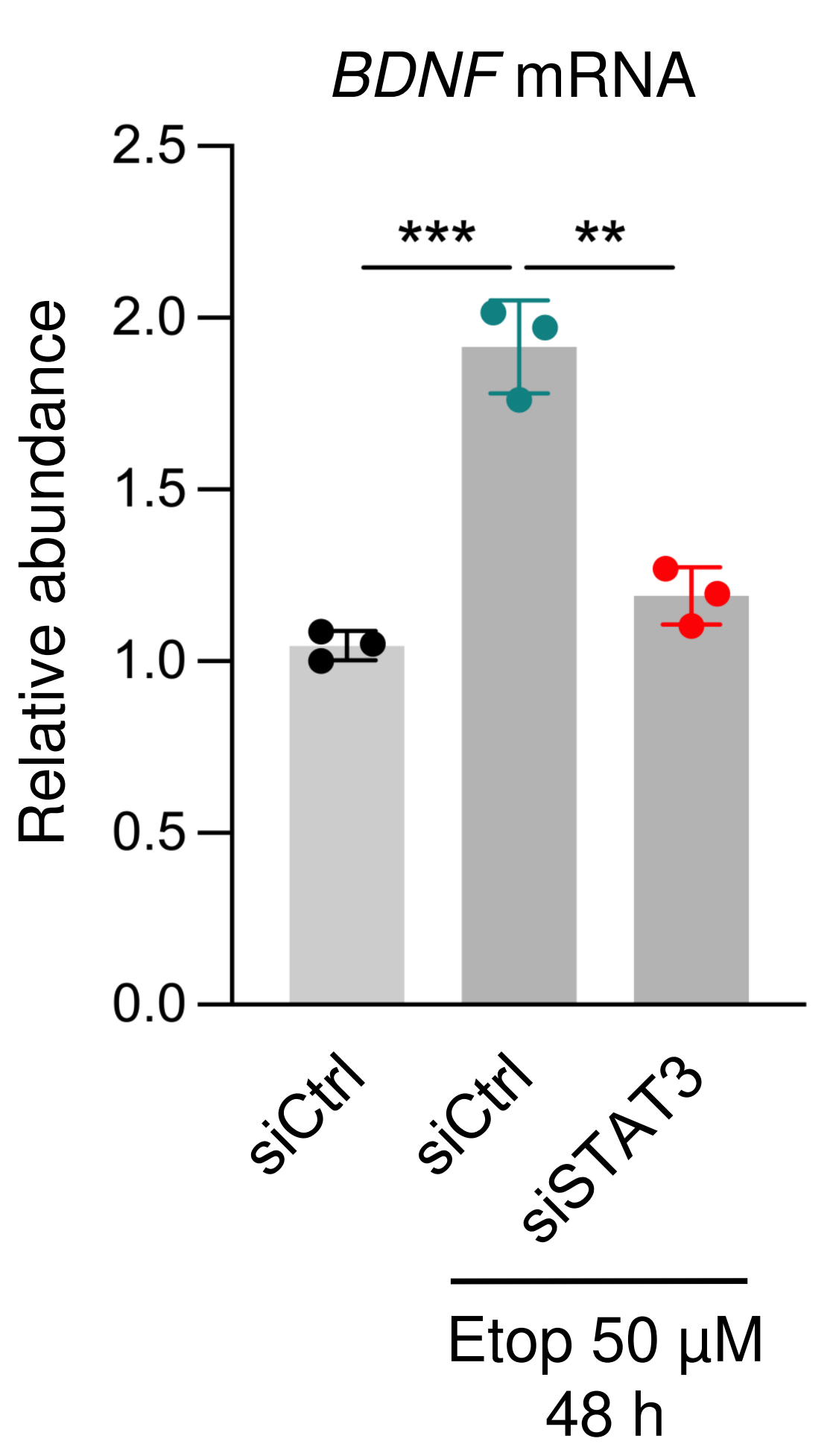

g

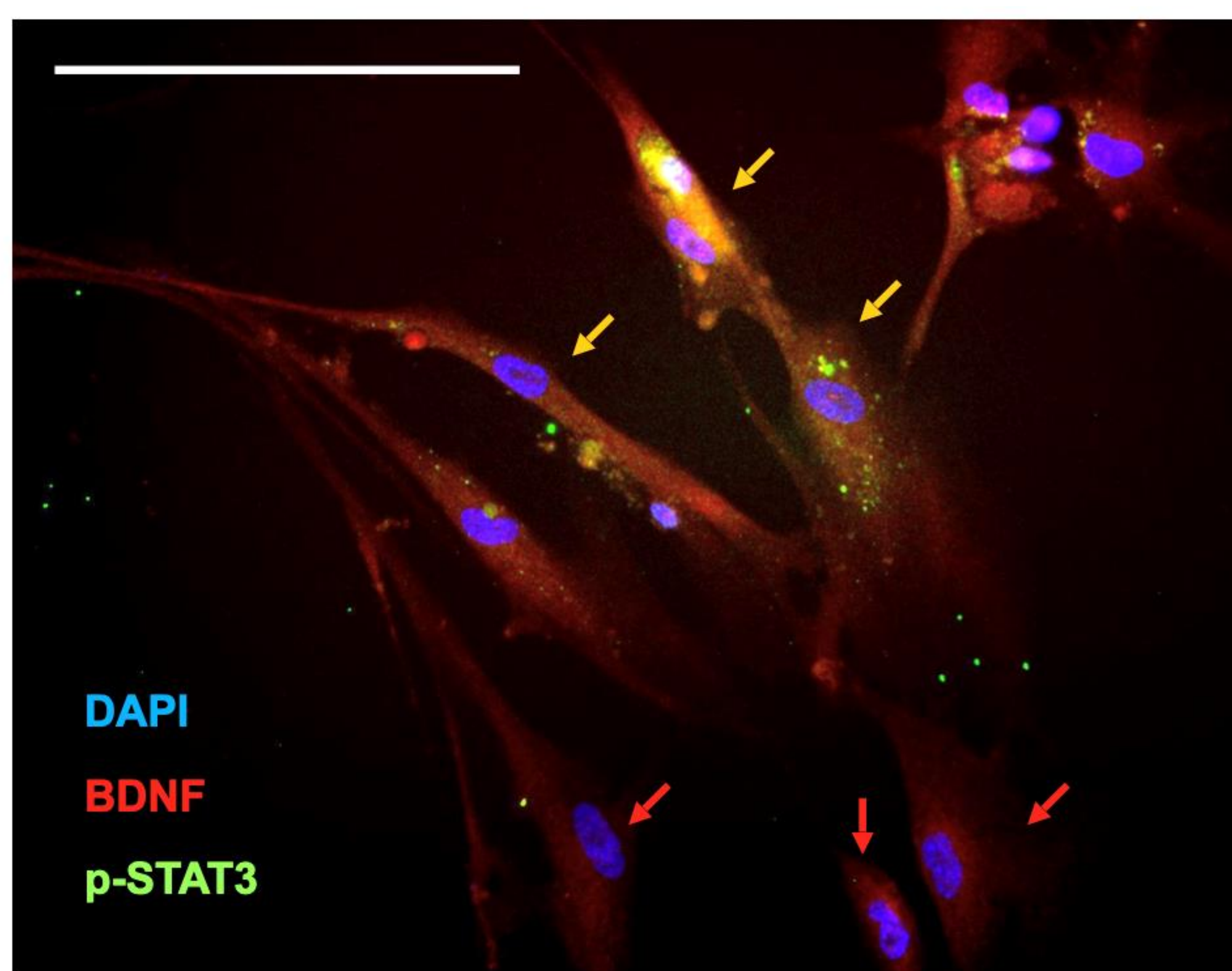

b

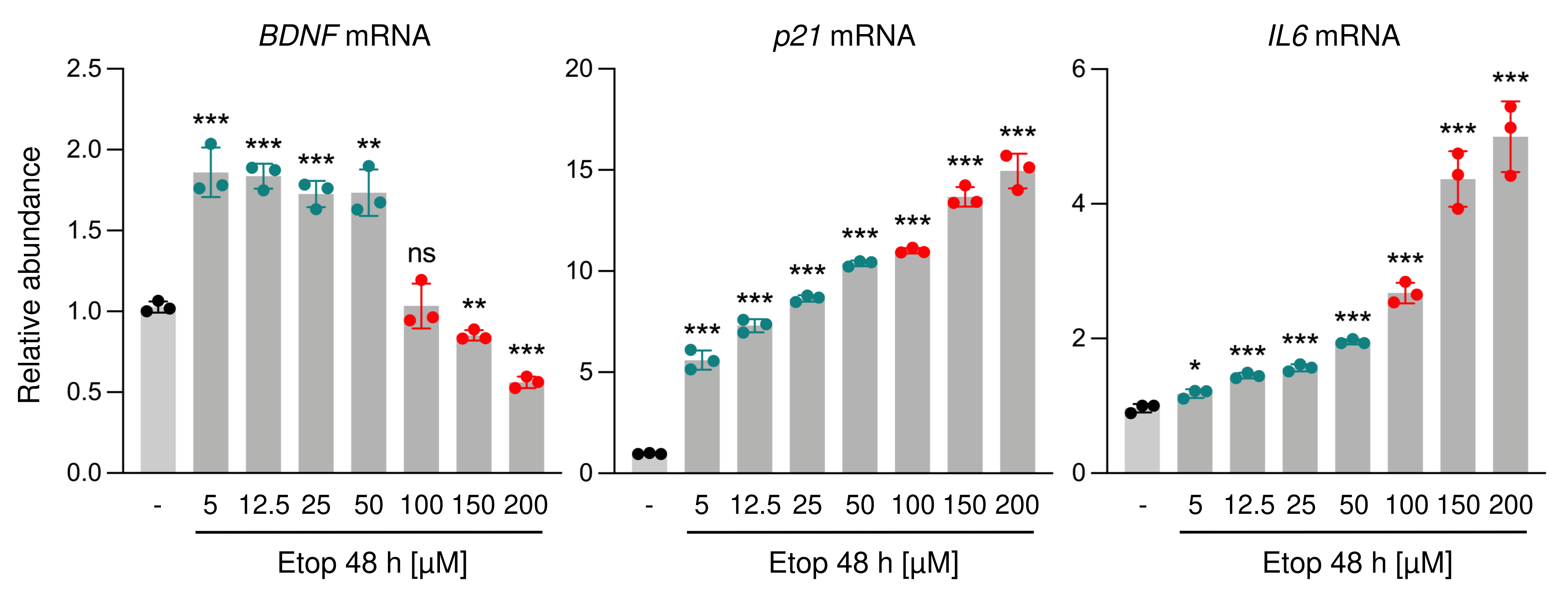

C

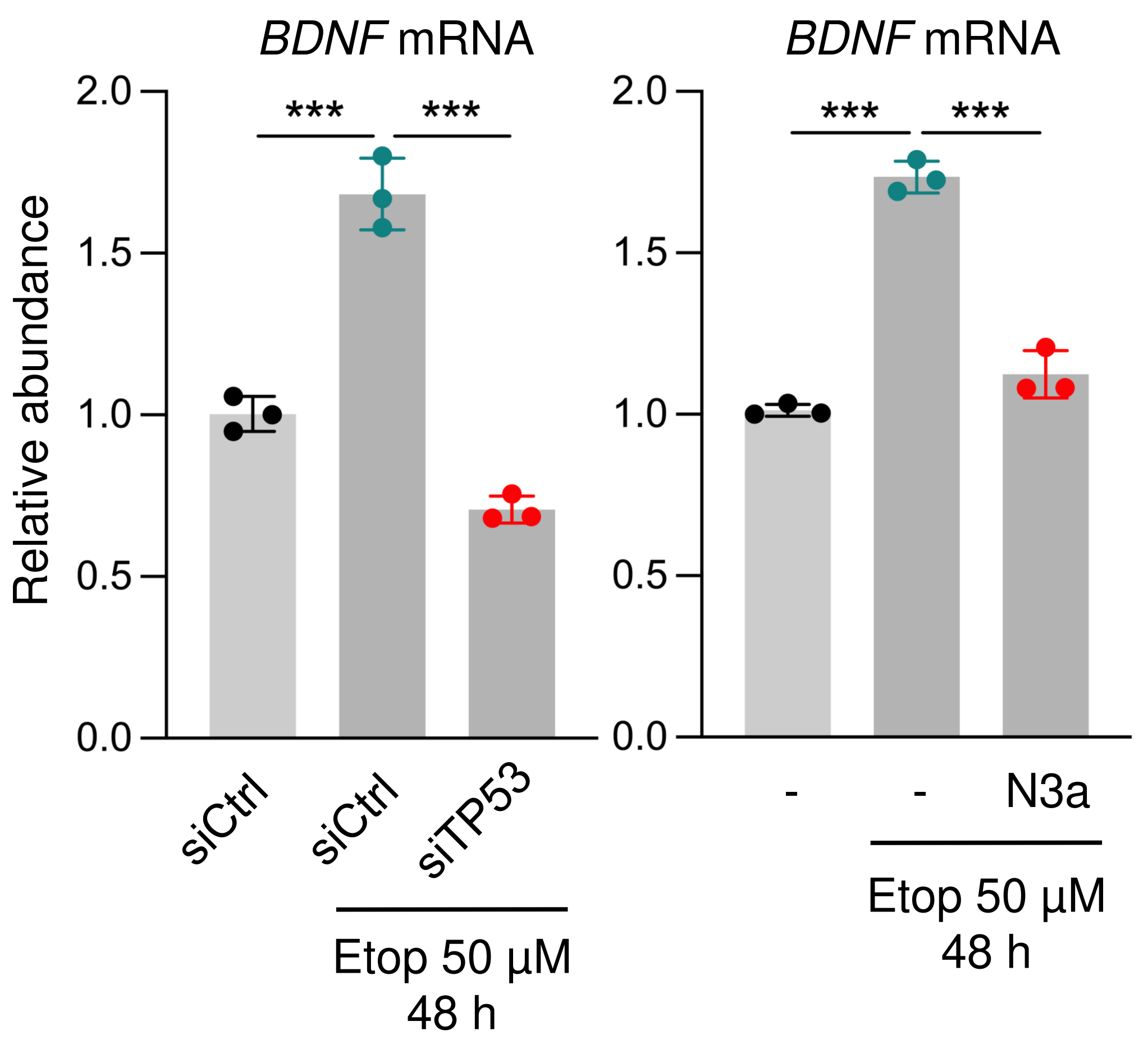

f

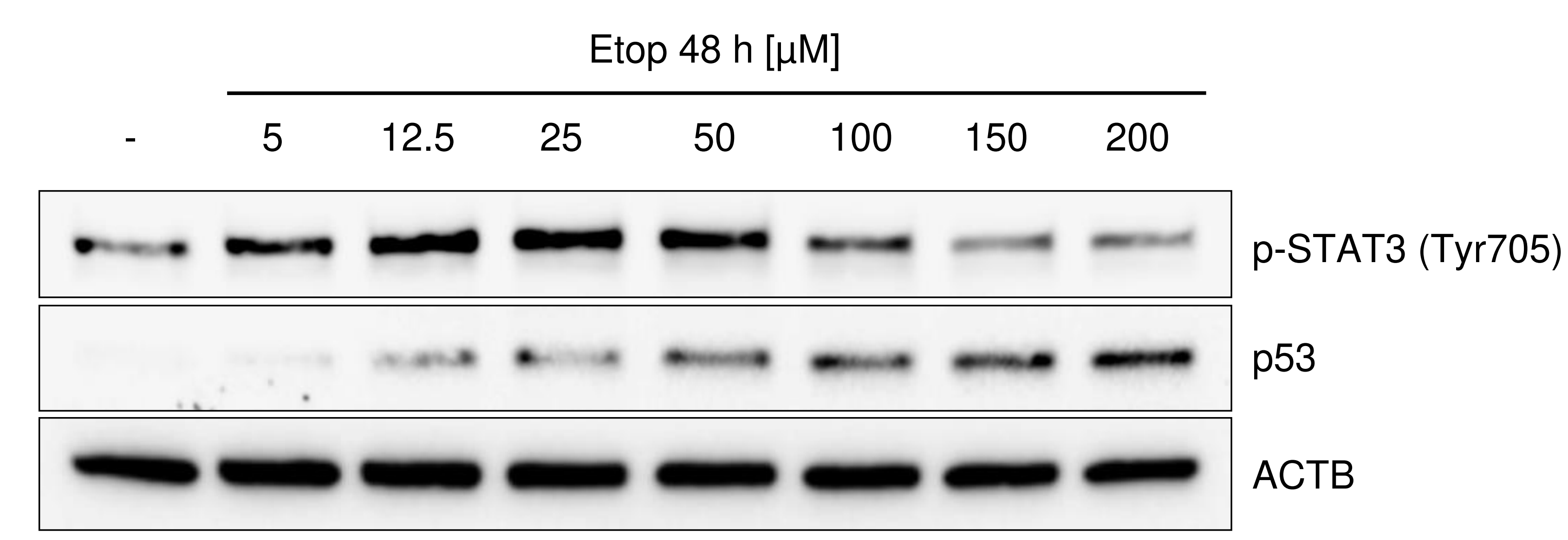

h

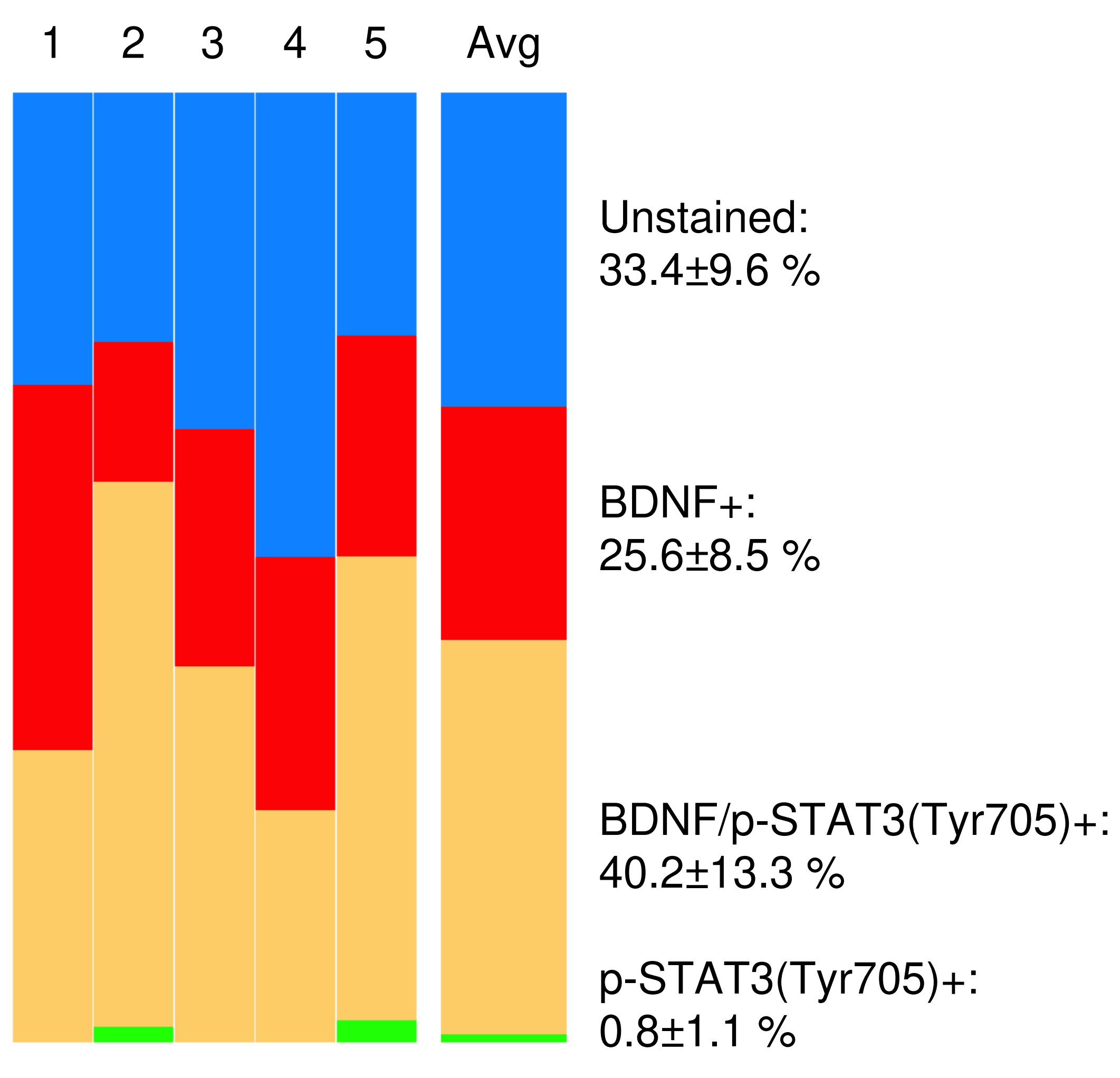

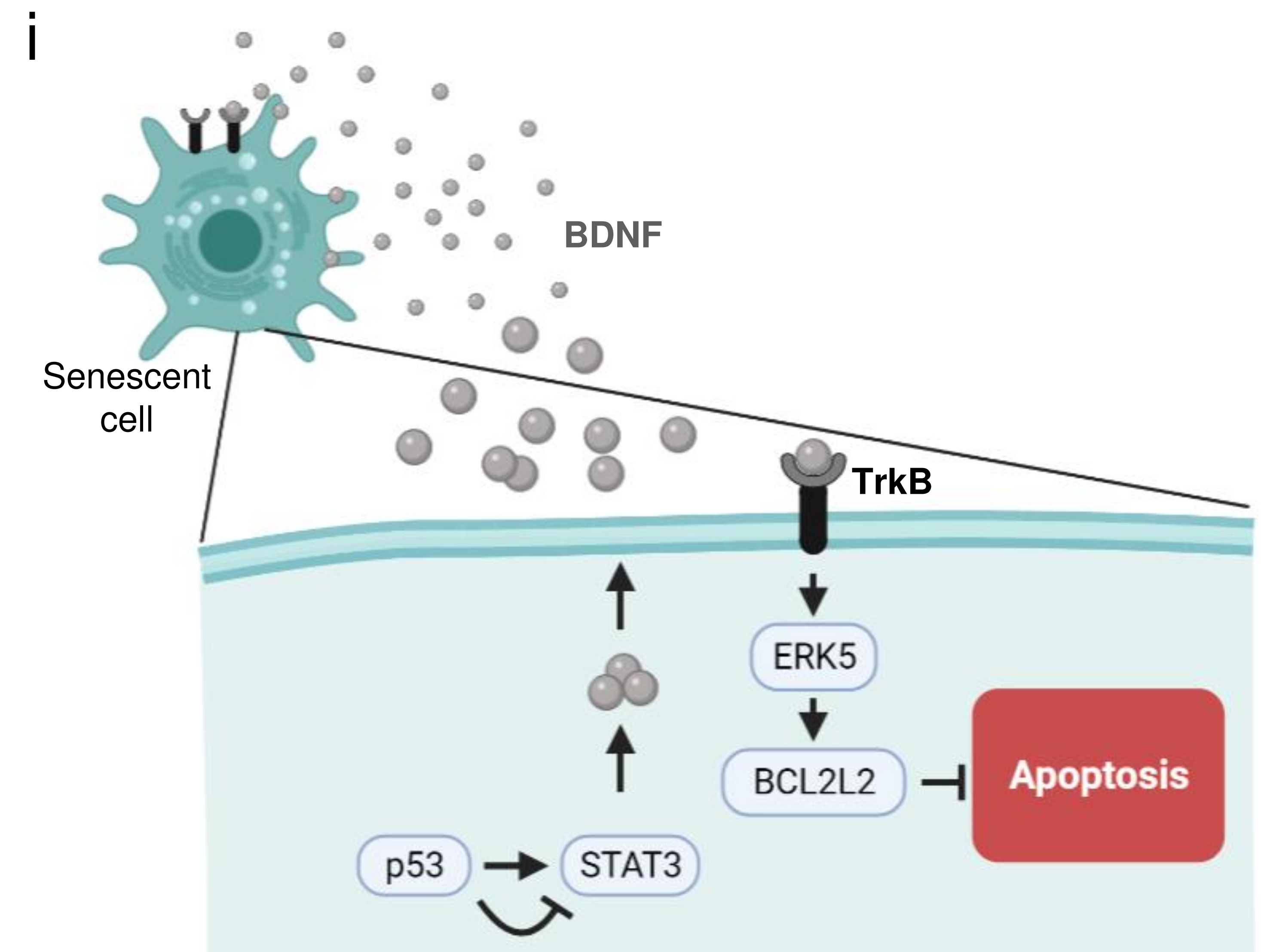


a

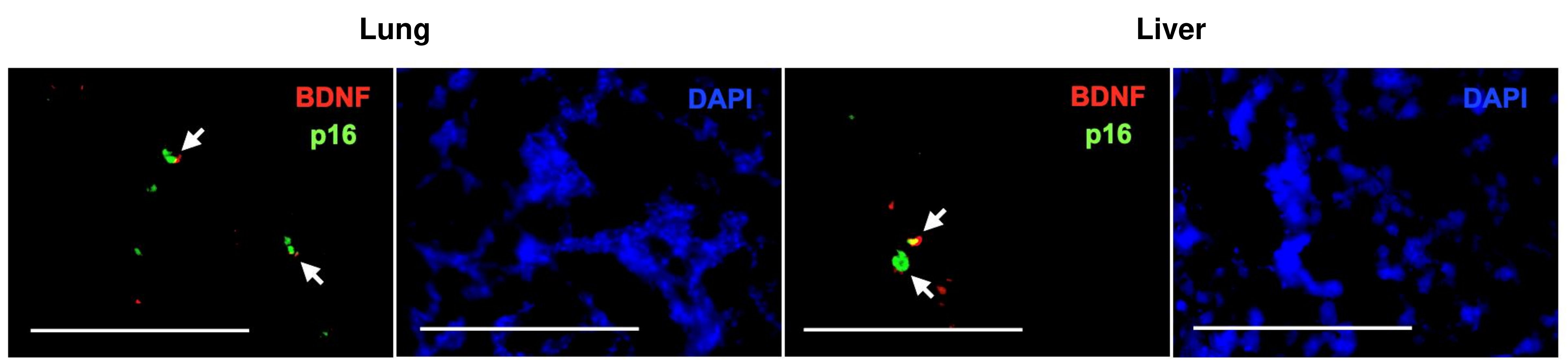

b

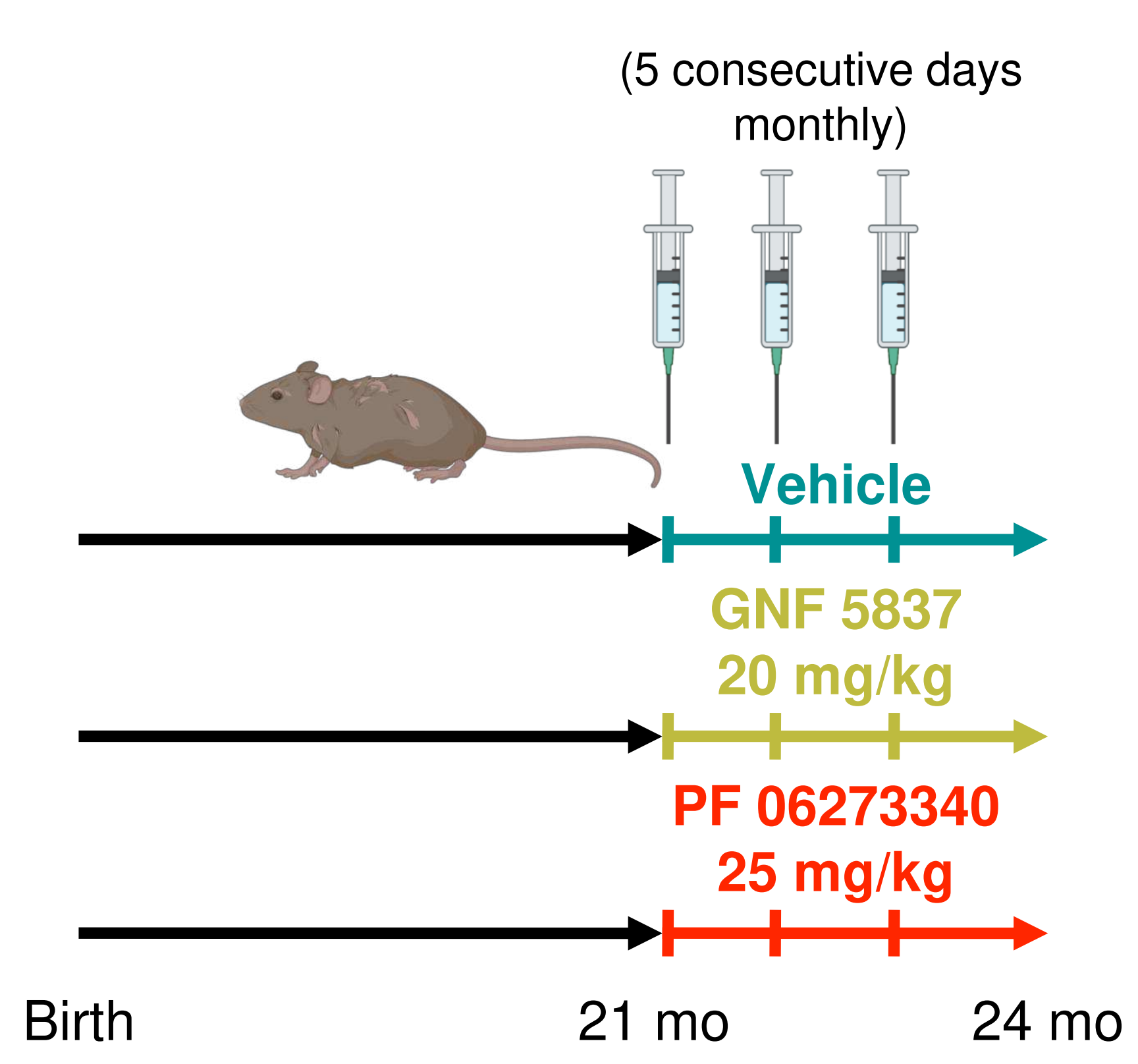

C

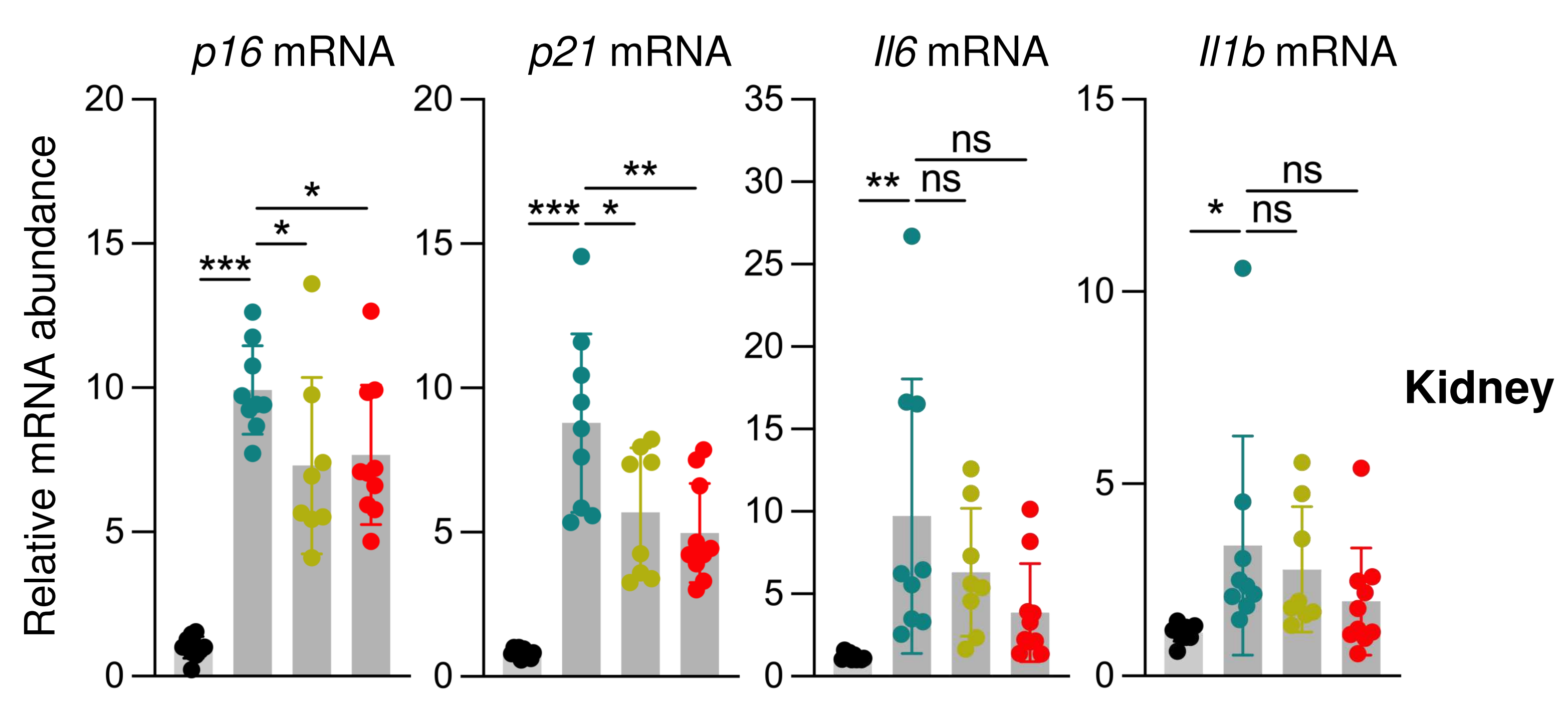

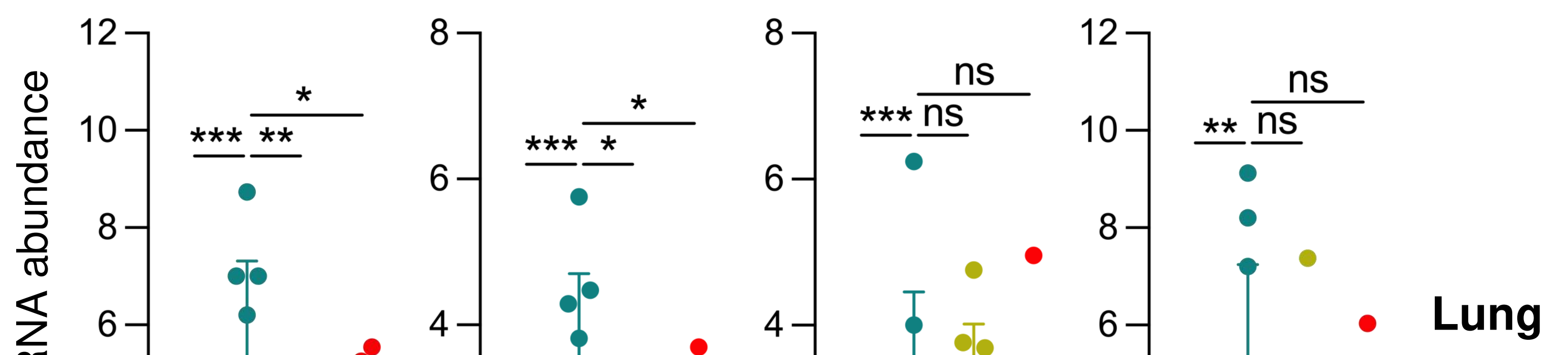

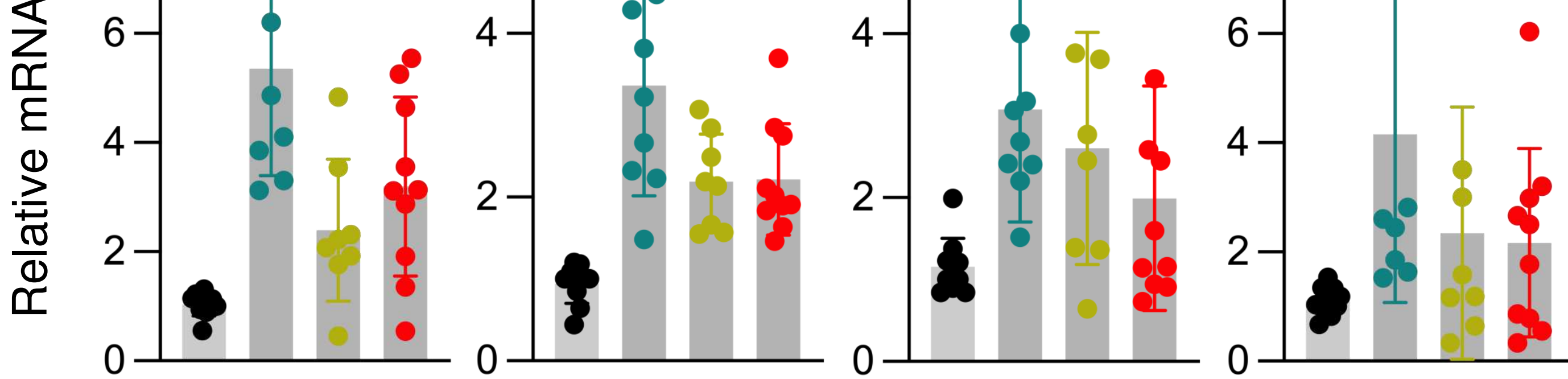

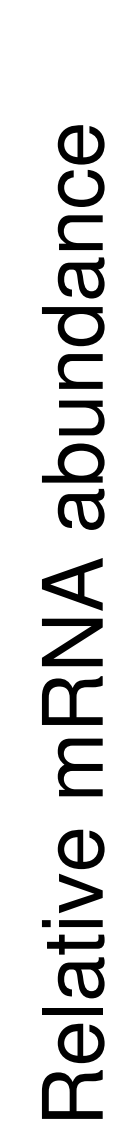

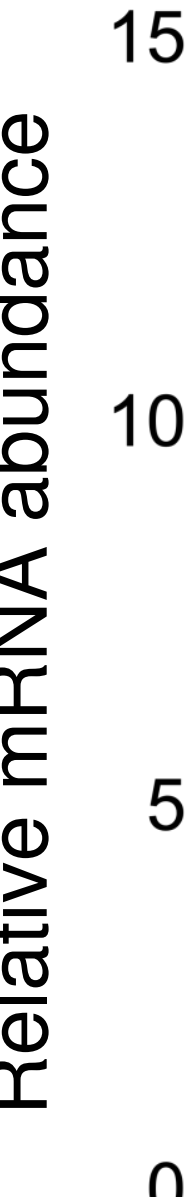

$\left.\begin{array}{l}15 \\ 10- \\ 5 \\ 0\end{array}\right]$

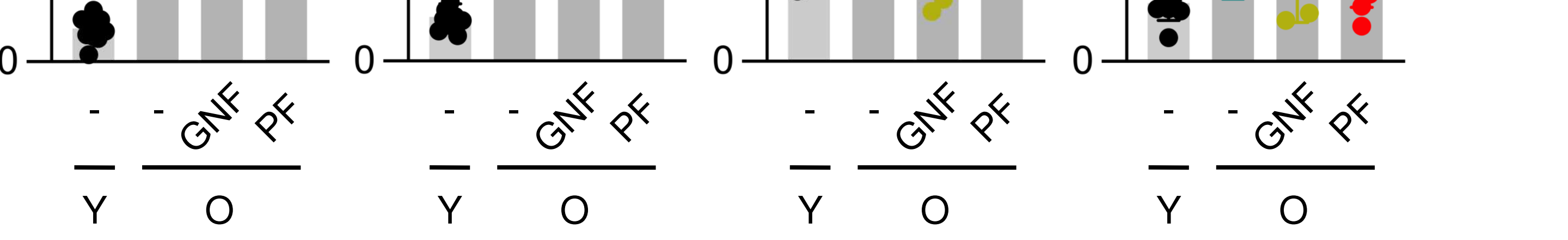

d

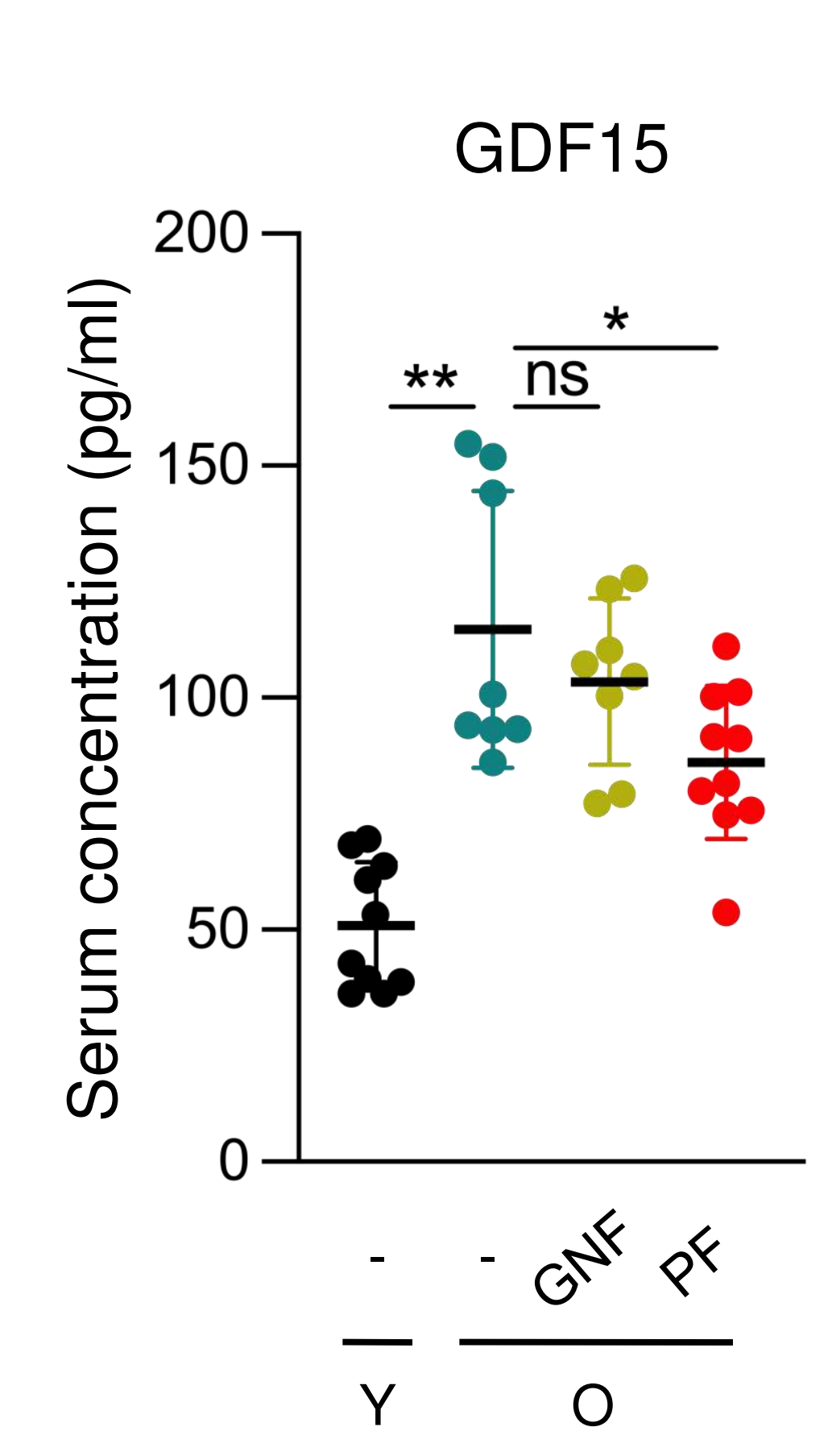

e

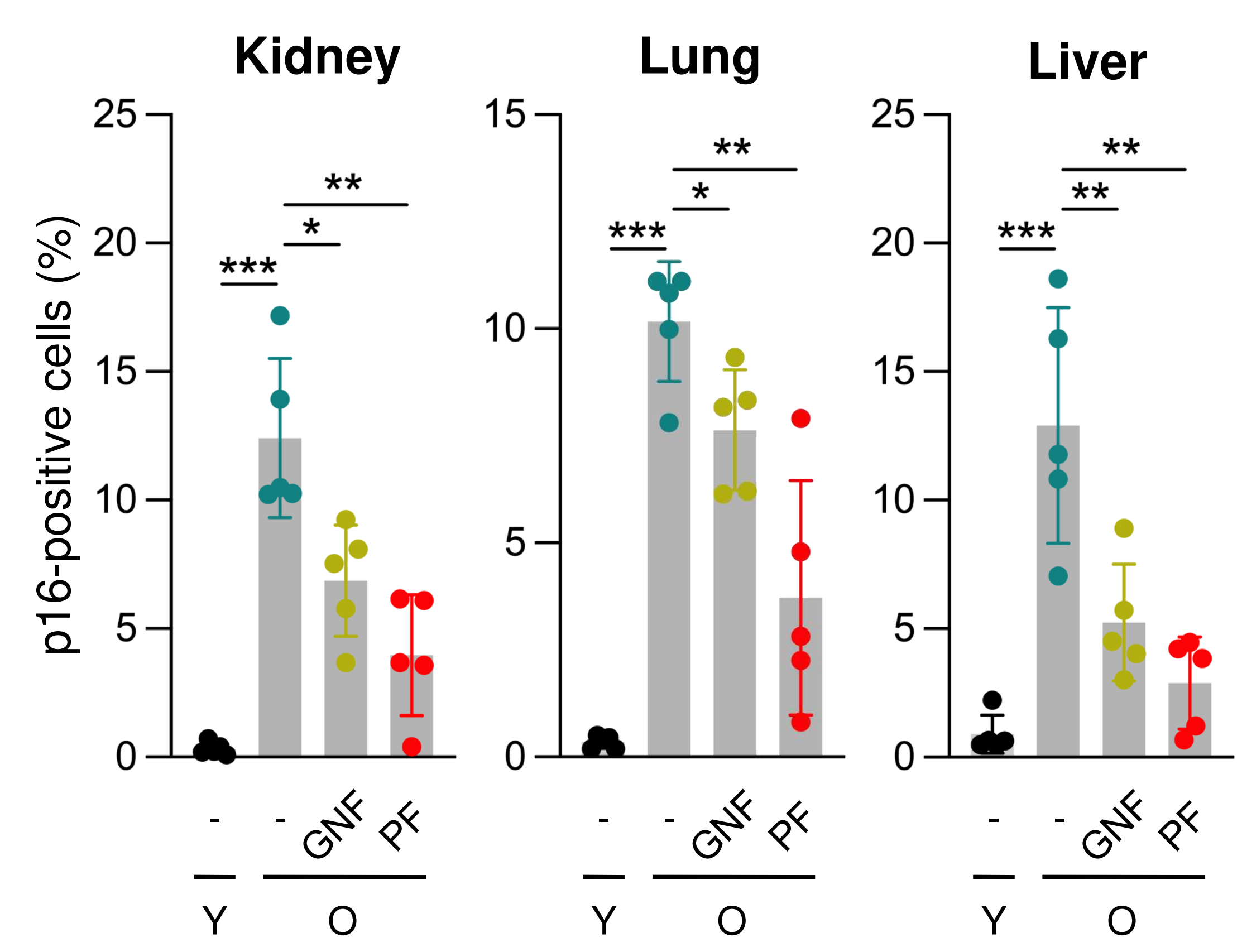

Old

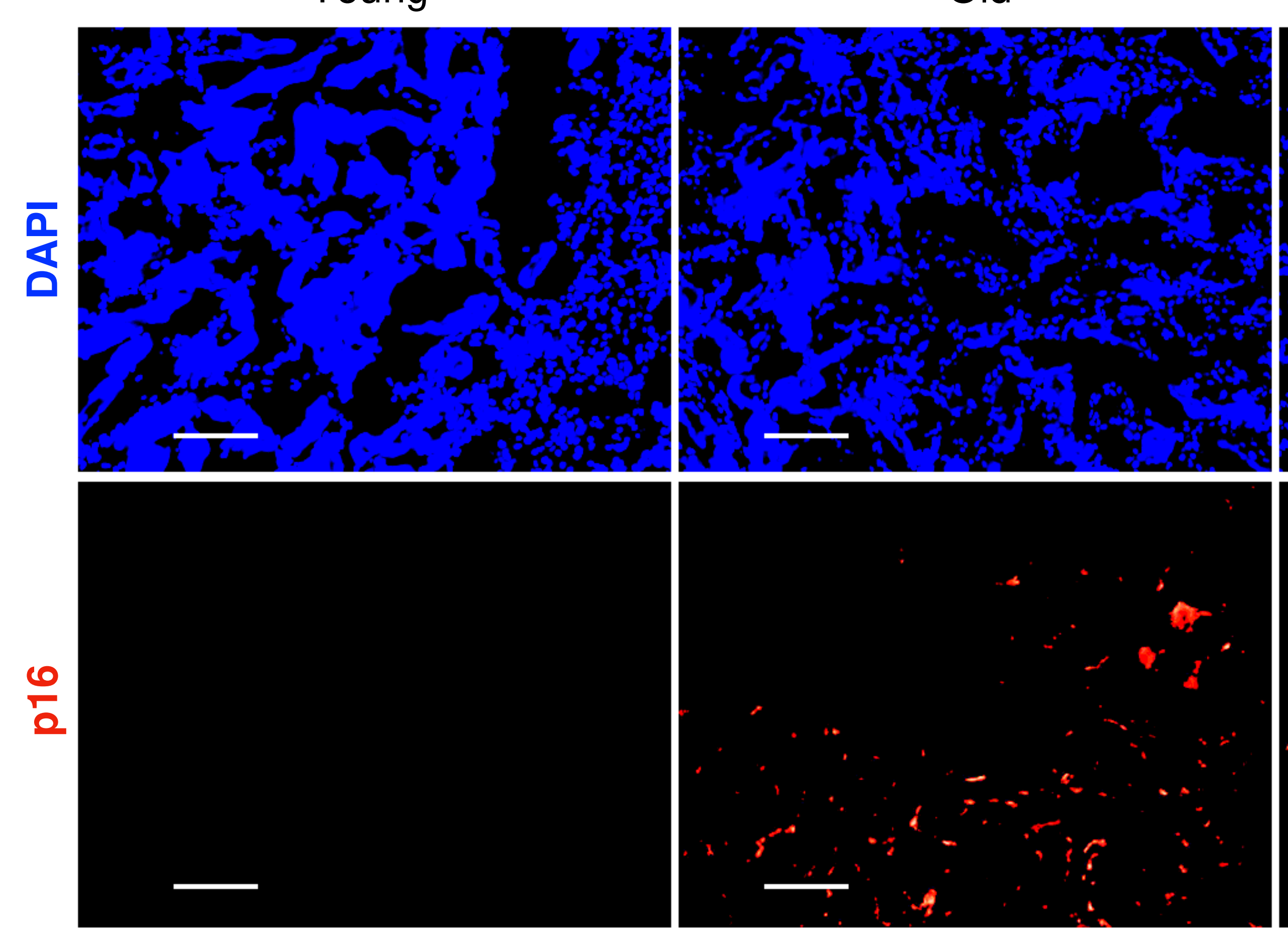

Old GNF 5837

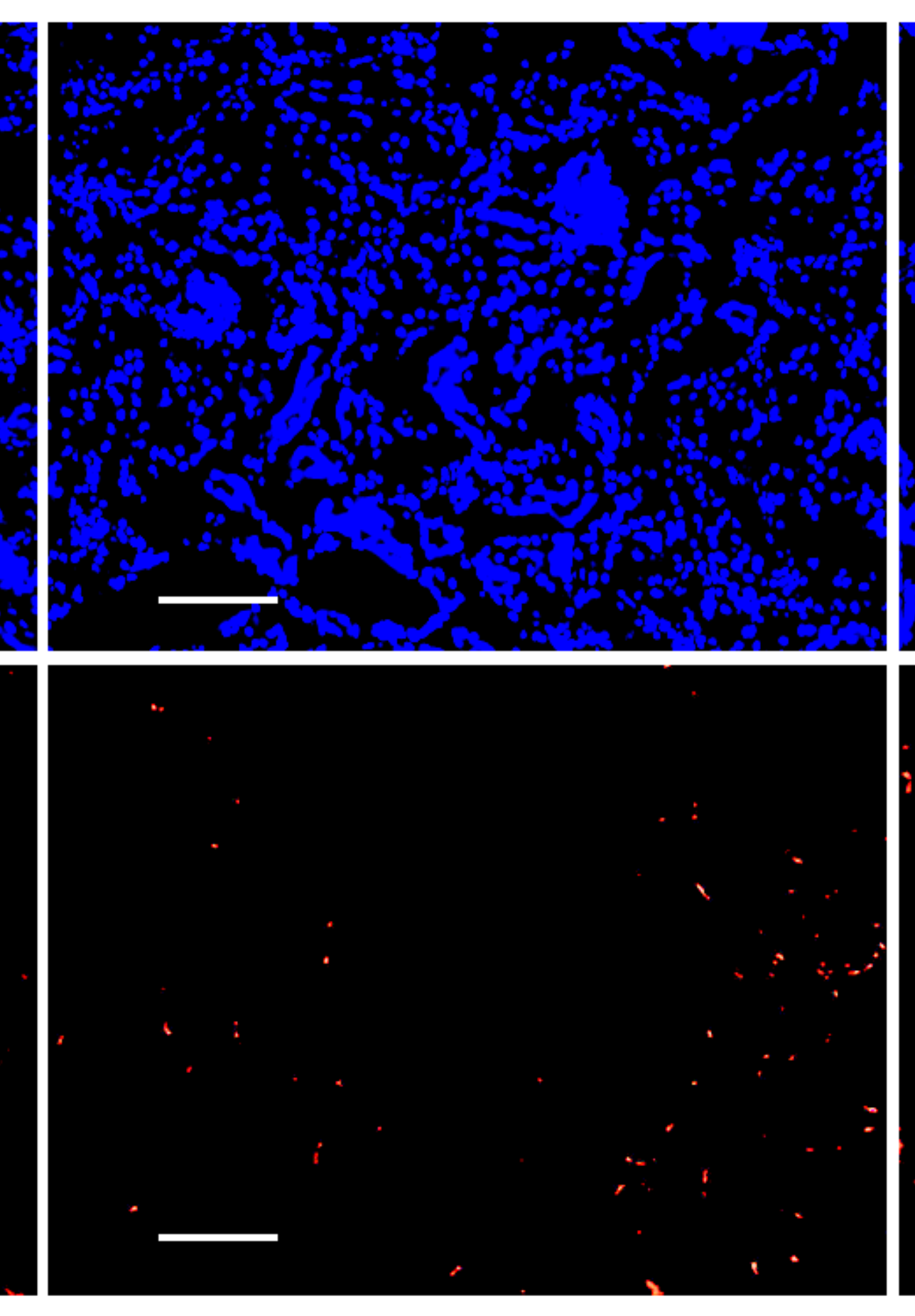

Old PF 06273340

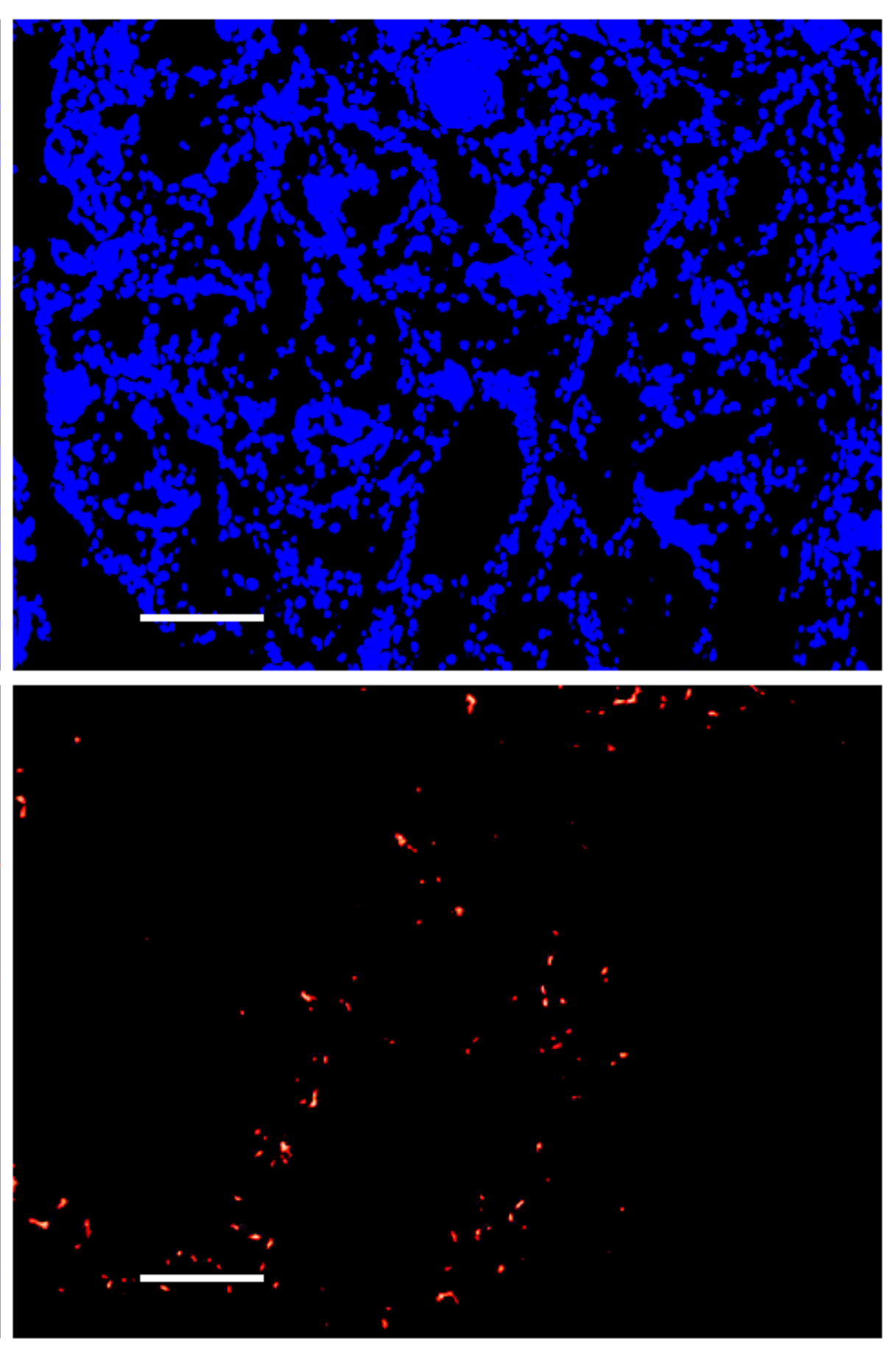

Kidney g

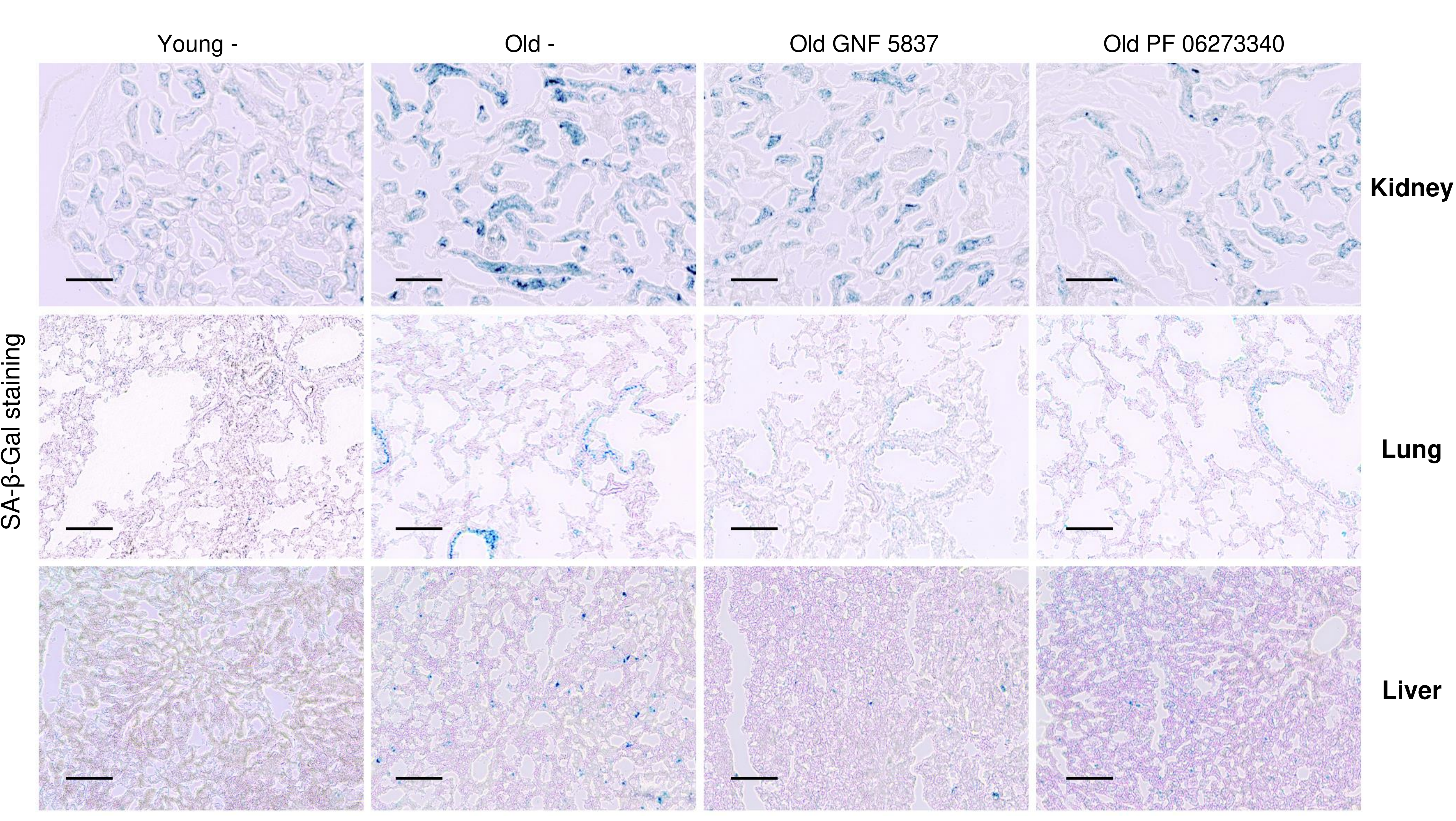

h

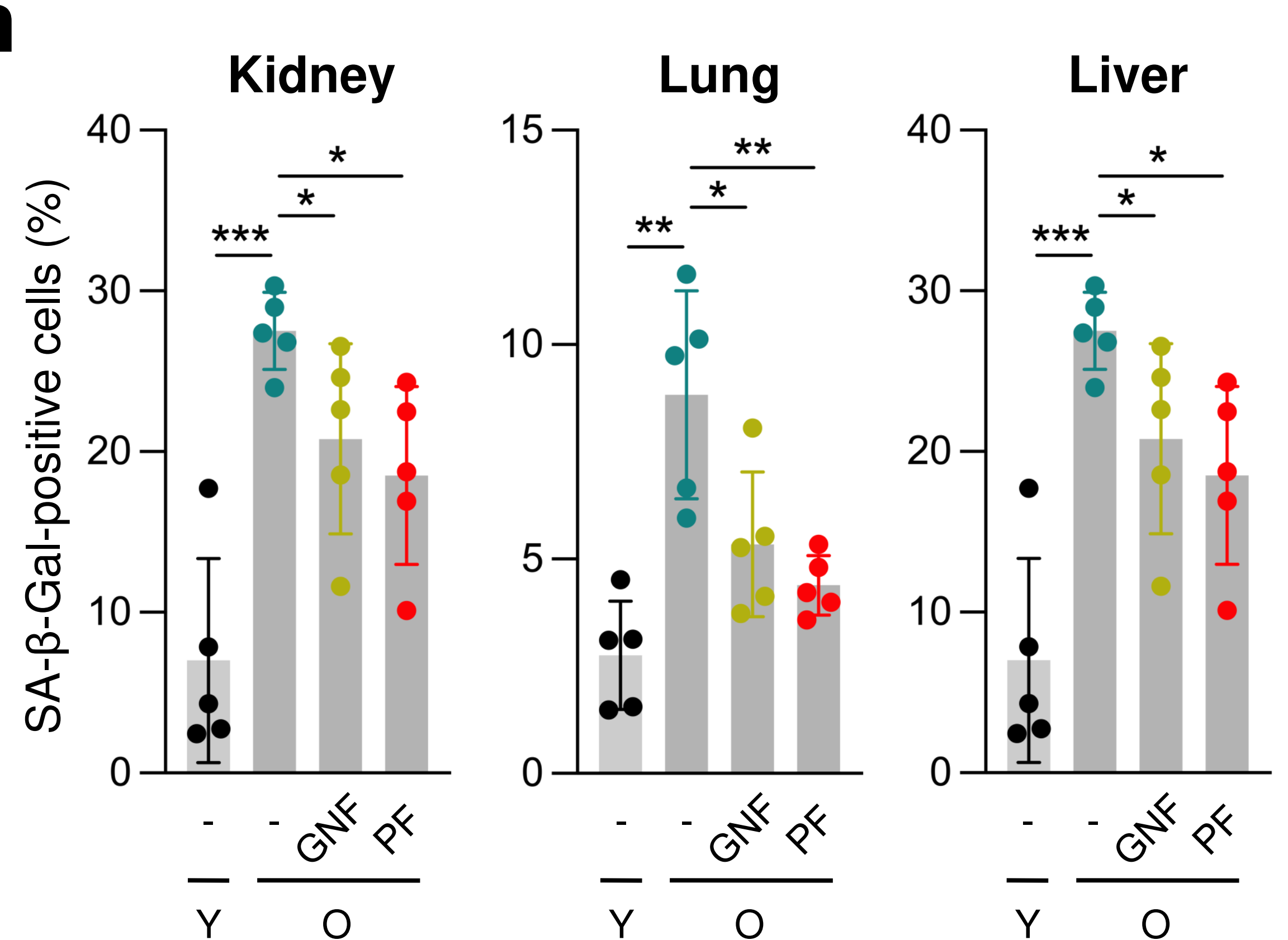




\section{Supplementary Files}

This is a list of supplementary files associated with this preprint. Click to download.

- Anerillasetal.SupplementaryTableS1.xIsx

- Anerillasetal.SupplementalTextandFigurescombined.pdf 